\title{
Dispersed Droplet Dynamics During Produced Water Treatment in Oil Industry
}

\author{
D.F. van Eijkeren
}


Dispersed Droplet Dynamics During Produced Water Treatment in Oil Industry D.F. van Eijkeren

Printed by Gildeprint

Cover: A schematic example of a tangential swirling flow separator

ISBN: 978-90-365-4043-8

DOI: $10.3990 / 1.9789036540438$

http://dx.doi.org/10.3990/1.9789036540438 


\title{
DISPERSED DROPLET DYNAMICS DURING PRODUCED WATER TREATMENT IN OIL INDUSTRY
}

\section{PROEFSCHRIFT}

\author{
ter verkrijging van \\ de graad van doctor aan de Universiteit Twente, \\ op gezag van de rector magnificus, \\ prof. dr. H. Brinksma, \\ volgens besluit van het College voor Promoties \\ in het openbaar te verdedigen \\ op vrijdag 22 januari 2016 om 12.45 uur
}

door

Diederik Floris van Eijkeren

geboren op 1 juli 1984

te Leidschendam 
Dit proefschrift is goedgekeurd door

promotor: prof. dr. ir. H. W. M. Hoeijmakers 


\section{ABstract}

For Lagrangian particle tracking applied to swirling flow produced water treatment the influence of the history force is investigated. In the expression for the history force an existing Reynolds number dependent kernel is adapted and validated for a range of experimental data for settling spheres. This kernel is used during the numerical simulation of oil-droplets in an idealized flow field as model for flow fields observed in swirling flow separators. It is shown that the history force should not be neglected for the motion of particles in such flows. However, for this specific type of flow it is shown that the contribution of the history force might be approximated as a drag-like expression.

Subsequently, an efficient collision detection mechanism is developed to obtain collision detection with a work-load of $\mathcal{O}\left(N_{\mathrm{p}} \ln N_{\mathrm{p}}\right)$. The scheme is validated for settling spheres and applied to the swirling flow field used during the investigation of the influence of the history force.

Finally, two approaches for the lattice Boltzmann method, taking into account the equation of state for water, are presented. One approach utilizing a pressure corrected BGK approximation, the other a dense gas BBGKY approximation. A novel approach is used to obtain the macroscopic quantities and boundary conditions. It is shown that for modeling the flow of water around a particle an ideal-gas lattice Boltzmann method to simulate water is not capable of capturing all relevant details. However, modeling the flow with both proposed approaches, which take the equation of state into account, lead to similar results. 



\section{SAMENVATTING}

Dit onderzoek gaat in op de kracht op een deeltje nodig voor een Lagrangiaanse methode voor het voorspellen van deeltjesbanen, toegepast op het behandelen van geproduceerd water met oliedeeltjes met behulp van een roterende stroming. De bijdrage in de kracht gerelateerd aan de historie van de beweging van het deeltje wordt in detail behandeld. Een bestaande kernel, afhankelijk van het Reynoldsgetal, voor de integraal in deze bijdrage in de kracht is aangepast en gevalideerd aan de hand van experimentele data voor sedimenterende bollen uit de literatuur. Deze aangepaste kernel is toegepast bij het numerieke voorspellen van banen van oliedruppels in een geïdealiseerd stromingsveld, kenmerkend voor roterende-stromingsscheiders. Dit onderzoek laat zien dat de historiekracht niet verwaarloosd kan worden bij het berekenen van de baan van deeltjes in zulke stromingen. Voor een specifiek geval wordt getoond dat deze kracht benaderd kan worden met een uitdrukking die lijkt op die van de weerstandskracht.

Vervolgens gaat dit onderzoek in op een efficiënte methode om botsingen te detecteren in dergelijke numerieke berekeningen. De benodigde rekentijd van deze methode varieert als $\mathcal{O}\left(N_{\mathrm{p}} \ln N_{\mathrm{p}}\right)$. De methode is eerst gevalideerd voor sedimenterende bollen. Vervolgens is de methode toegepast en onderzocht in het bovengenoemde kenmerkende stromingsveld in roterende stromingsscheiders.

Tenslotte worden twee methodes gepresenteerd om de toestandsvergelijking van water te implementeren in de lattice Boltzmann methode. Eén methode gebruikt de BGK-benadering gecombineerd met een drukcorrectie, terwijl de andere methode de BBGKY-benadering voor een zwaar gas gebruikt. Een alternatieve methode is toegepast om de macroscopische grootheden te verkrijgen en de randvoorwaarden op te leggen. Er wordt aangetoond dat een methode met een ideaal gas voor de lattice Boltzmann methode niet in staat is om alle relevante details goed te voorspellen. Beide voorgestelde methoden die de toestandsvergelijking van water mee nemen leiden tot significant andere voorspellingen, terwijl hun resultaten onderling nauwelijks verschillen. 



\title{
Contents
}

\begin{abstract}
$\quad$ v
Samenvatting vii

Contents ix

$\begin{array}{ll}\text { Nomenclature } & \text { xiii }\end{array}$
\end{abstract}

1 Introduction $\quad \mathbf{1}$

1.1 Introduction to oil-water separation methods . . . . . . . . . . 2

1.1.1 Swirling flow separation . . . . . . . . . . . . 2

1.1.2 Bubble enhanced separation methods . . . . . . . . . 3

1.1.3 Lamellar settling and flotation . . . . . . . . . . . . 5

1.1.4 Efficient formation of flocs . . . . . . . . . . . 5

1.1.5 Surface chemistry important for flotation . . . . . . . . 6

1.1.6 De-gassing reduces coalescence . . . . . . . . . . 7

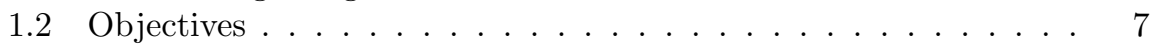

1.3 Outline ....................... 7

2 Particle motion $\quad 11$

2.1 Equations of motion . . . . . . . . . . . . . . 11

2.1.1 Linear momentum and position . . . . . . . . . . . . 12

2.1.2 Angular momentum . . . . . . . . . . . . . . . . 14

2.2 System of differential equations . . . . . . . . . . . . . 16

3 Force and torque $\quad 17$

3.1 Approximation of the force and torque . . . . . . . . . . . 17

3.2 Dimensionless and characteristic numbers . . . . . . . . . . 18 
3.3 Volume force and torque . . . . . . . . . . . . . . . . . 21

3.3.1 Particle of an arbitrary shape . . . . . . . . . . . 21

3.3.2 Sphere of uniform density . . . . . . . . . . . . . . . 22

3.4 Stress divergence force and torque . . . . . . . . . . . . 23

3.4.1 Symmetric stress matrix for an arbitrary particle . . . . 25

3.4.2 Symmetric stress matrix for a sphere of uniform density . 25

3.4 .3 Buoyancy force . . . . . . . . . . . . . . . 26

3.5 Added mass force . . . . . . . . . . . . . . . . . . . 26

3.6 Lift forces . . . . . . . . . . . . . . . . . . . . . 27

3.6.1 Saffman lift . . . . . . . . . . . . . . . . . 28

3.6 .2 Magnus lift . . . . . . . . . . . . . . . . 30

3.7 Drag force . . . . . . . . . . . . . . . . . . 32

3.7.1 Drag coefficient and drag factor . . . . . . . . . . . . . 32

3.7.2 Approximation of the drag force . . . . . . . . . . 34

3.7.3 Approximation of the torque due to drag . . . . . . . . 35

3.8 History force . . . . . . . . . . . . . . . . . . . . . 35

3.8.1 Basset kernel for the history force . . . . . . . . . . . . 36

3.8.2 Influence of inertia on the history kernel . . . . . . . . . . 36

3.8.3 Approximation of the history force and torque . . . . . . 39

3.8.4 Dealing with minus infinity . . . . . . . . . . . . . . 39

3.9 First approximation for force and torque . . . . . . . . . . . 41

4 Numerical treatment $\quad 43$

4.1 Discretized equation of motion . . . . . . . . . . . . . 44

4.2 Numerical integration for the history force . . . . . . . . . . . . . . 45

4.2.1 The general history kernel . . . . . . . . . . . . . . . 45

4.2.2 Basset kernel with constant time-step . . . . . . . . . . . 46

4.3 Incorporation of the discrete history integral . . . . . . . . . . . 48

5 Settling sphere $\quad 49$

5.1 Flow and particle attributes . . . . . . . . . . . . . . 49

5.2 Results and discussion . . . . . . . . . . . . . . 50

6 Oil droplet in swirling flow $\quad \mathbf{5 7}$

6.1 Swirling flow and particle attributes . . . . . . . . . . 57

6.1 .1 Swirling flow field . . . . . . . . . . . . 57

6.1 .2 Fluid and particle properties ............ 60

6.2 Results and discussion . . . . . . . . . . . . . . . . 6 60 60

6.2.1 Non-Stokesian effects, history force and lift . . . . . . . . 60 
6.2.2 An efficient approximation of the history force . . . . . 64

$\begin{array}{lll}7 & \text { Efficient collision detection } & 69\end{array}$

7.1 Collision detection scheme . . . . . . . . . . . . 70

7.1.1 Straightforward implementations . . . . . . . . . . 70

7.1 .2 Proposed implementation . . . . . . . . . . . . . . 71

7.2 Collision detection for settling spheres . . . . . . . . . . . 72

7.2.1 Material properties and geometry . . . . . . . . . 74

7.2 .2 Results and discussion . . . . . . . . . . . . . . 76

7.3 Collision detection in swirling flow . . . . . . . . . . . . 79

7.3 .1 Results and discussion . . . . . . . . . . . . . 80

8 Lattice Boltzmann for water $\quad 83$

8.1 From molecular to macroscopic . . . . . . . . . . . . . . . . 83

8.2 The Boltzmann equation . . . . . . . . . . . . . . . 86

8.3 Approximation to the collision term . . . . . . . . . 87

8.3 .1 BGK approximation . . . . . . . . . . . 87

8.3.2 BBGKY approximation . . . . . . . . . . . . . . 88

8.4 Incorporating an equation of state for water . . . . . . . . . . 89

8.4.1 BGK approximation . . . . . . . . . . . . 90

8.4 .2 BBGKY approximation . . . . . . . . . . . . . . 91

8.4 .3 Viscosity of water . . . . . . . . . . . . . 92

8.5 Approximation for $\nabla_{c} f \ldots \ldots \ldots 9 . \ldots \ldots$

8.5.1 BGK approximation for water . . . . . . . . . 94

8.5.2 BBGKY approximation for water . . . . . . . . . . . 94

8.6 Discretized velocity space . . . . . . . . . . . . . . . 96

8.7 Space-time lattice discretization . . . . . . . . . . . . . . . . . . . . . . . . . . . . . . . . . . .

8.8 Boundary conditions . . . . . . . . . . . . . . . . . . . . 104

8.9 Simulation set-up . . . . . . . . . . . . . . . . 106

8.10 Results and discussion . . . . . . . . . . . . . . . 107

9 Concluding remarks and recommendations 113

9.1 Faxèn forces, lift forces and torque . . . . . . . . . . . . . . 113

9.2 The history force and its influence . . . . . . . . . . . . . . . 114

9.3 Efficient collision detection . . . . . . . . . . . . . . 115

9.4 A lattice Boltzmann method for water . . . . . . . . . . . 116

$\begin{array}{lr}\text { Bibliography } & 119\end{array}$ 
A Derivations related to the equations of motion $\quad \mathbf{1 2 5}$

A.1 Particle postion . . . . . . . . . . . . . . . . 125

A.2 Particle rate of rotation . . . . . . . . . . . . . 128

$\begin{array}{lr}\text { B Integrals for spherical volume } & 131\end{array}$

B.1 Volume of a sphere . . . . . . . . . . . . . . . . . . . 131

B.2 From Cartesian to spherical . . . . . . . . . . . . . . . . 132

B.3 Surface of a sphere . . . . . . . . . . . . . . . . . . . 134

B.4 First moment for a sphere . . . . . . . . . . . . . . . . . . 134

B.5 Second moment for a sphere . . . . . . . . . . . . . 135

B.6 Moment of inertia . . . . . . . . . . . . . . . 137

B.6.1 A uniform sphere . . . . . . . . . . . . . . . 137

B.6.2 A uniform spherical shell . . . . . . . . . . . . . 138

B.6.3 A uniform sphere with a uniform shell . . . . . . . . . . 139

B.7 Fourth moment for a sphere . . . . . . . . . . . . . . . 139

B.8 List of moments . . . . . . . . . . . . . . . . . . . 141

$\begin{array}{lr}\text { Acknowledgements } & 143\end{array}$

$\begin{array}{ll}\text { About the author } & 145\end{array}$ 


\section{NomenClature}

\section{Fonts}

Font Description

$a, A, \alpha \quad$ Scalar

$\boldsymbol{a}, \boldsymbol{A}, \boldsymbol{\alpha} \quad$ Vector

a, A, $\alpha \quad$ Matrix

$\mathcal{A} \quad$ Characteristic quantity, or specific version of a quantity

\section{Roman symbols}

\section{Symbol Description}

a Attractive force factor

a Particle acceleration vector

a Arbitrary vector

$\mathcal{A} \quad$ Characteristic area

$b \quad$ Attractive force factor

$b \quad$ Molecular effective volume factor

c Absolute molecular velocity

c Molecular velocity vector

$C \quad$ Absolute peculiar velocity of molecule

$C \quad$ Peculiar velocity vector of molecule vector

$C \quad$ Coefficient in analytical or empirical relation

$D \quad$ Diameter

$\mathcal{D} \quad$ Number of dimensions

$f \quad$ Factor in analytical or empirical relation

$f \quad$ Probability density function

$f \quad$ Volumetric, or body, force vector 
$\begin{array}{ll}F & \text { Magnitude force } \\ \boldsymbol{F} & \text { Force vector } \\ \mathcal{F} & \text { Characteristic force magnitude }\end{array}$

$\left[\mathrm{kg} \mathrm{m \textrm {s } ^ { - 2 }}\right]$ $\left[\mathrm{kg} \mathrm{ms}^{-2}\right]$ $\left[\mathrm{kg} \mathrm{ms}^{-2}\right]$ Absolute gravitational acceleration

$\left[\mathrm{m} \mathrm{s}^{-2}\right]$ Gravitational acceleration vector

$\left[\mathrm{ms} \mathrm{s}^{-2}\right]$

g

$h$ height

$\mathcal{H}_{\rho} \quad$ Non-ideal density force term for LBM

$\mathcal{H}_{T} \quad$ Non-ideal temperature force term for LBM

$\mathcal{H}_{\boldsymbol{u}} \quad$ Non-ideal velocity force term for LBM

Summation counter

I Moment of inertia matrix

I Scalar moment of inertia

$k \quad$ Boltzmann constant: $1.3806488 \times 10^{-23}\left[\mathrm{~J} \mathrm{~K}^{-1}\right]$

$K \quad$ History kernel

$\mathcal{L} \quad$ Characteristic length

$m \quad$ Particle/molecule mass

$n \quad$ Number density

$\boldsymbol{n} \quad$ Outward unit normal vector

$\mathcal{N} \quad$ Characteristic kinematic viscosity

$\mathcal{O} \quad$ Of the order of

$\mathcal{O} \quad$ Vector with elements of the order of

$p \quad$ Pressure

$p \quad$ Arbitrary integer power

$\mathcal{P} \quad$ Probability function

$\boldsymbol{r} \quad$ Position vector in spherical coordinate system

$R \quad$ Radius

$\mathcal{R} \quad$ Characteristic density

Re Reynolds number

$s \quad$ Integration time

$S \quad$ Surface

$t \quad$ Time

$T \quad$ Temperature

$\boldsymbol{T}$ Torque

$\mathcal{T} \quad$ Characteristic torque

$u \quad$ Absolute velocity

$\boldsymbol{u} \quad$ Velocity vector

$\mathcal{U} \quad$ Characteristic velocity

U Unit matrix

$[\mathrm{m}]$

$\left[\mathrm{kg}^{-1} \mathrm{~m}^{2} \mathrm{~s}^{-2}\right]$ $\left[\mathrm{m}^{2} \mathrm{~s}^{-2} \mathrm{~K}^{-1}\right]$ $\left[\mathrm{m} \mathrm{s}^{-2}\right]$

$\left[\mathrm{kg} \mathrm{m}^{2}\right]$ $\left[\mathrm{kg} \mathrm{m}^{2}\right]$

$\left[\mathrm{kg} \mathrm{m}^{2} \mathrm{~s}^{-2} \mathrm{~K}^{-1}\right]$

$\left[\mathrm{kg} \mathrm{m}^{-1} \mathrm{~s}^{-2}\right]$ $\left[\mathrm{m}^{-3} \mathrm{~s}^{3}\right]$ [m] $\left[\mathrm{kg} \mathrm{m}^{-3}\right]$ 
$v \quad$ Magnitude particle velocity

$\boldsymbol{v} \quad$ Particle velocity vector

$V \quad$ Volume

$\boldsymbol{w} \quad$ Relative particle velocity vector

$x \quad$ First component of $\boldsymbol{x}$

$\boldsymbol{x} \quad$ Position vector in Cartesian coordinate system

Second component of $\boldsymbol{x}$

Third component of $\boldsymbol{x}$

$$
\begin{array}{r}
{\left[\mathrm{ms}^{-1}\right]} \\
{\left[\mathrm{m} \mathrm{s}^{-1}\right]} \\
{\left[\mathrm{m}^{3}\right]} \\
{\left[\mathrm{m} \mathrm{s}^{-1}\right]} \\
{[\mathrm{m}]} \\
{[\mathrm{m}]} \\
{[\mathrm{m}]} \\
{[\mathrm{m}]}
\end{array}
$$

\section{Greek symbols}

\section{Symbol Description}

$\alpha \quad$ Short range repulsive force factor

$\Gamma \quad$ Circulation

$\vartheta \quad$ Second component of $\boldsymbol{r}$, polar angle

$\kappa \quad$ Heat conductivity

$\lambda \quad$ Short range repulsive factor

$\mu \quad$ Dynamic viscosity

$\nu \quad$ Kinematic viscosity

$\rho \quad$ Density

$\varrho \quad$ First component of $\boldsymbol{r}$, radial distance

$\boldsymbol{\sigma} \quad$ Stress matrix

$\tau \quad$ Timescale, relaxation time or integration time

$\phi \quad$ Massflux

$\varphi \quad$ Third component of $\boldsymbol{r}$, azimuthal angle

$\boldsymbol{\Phi} \quad$ Total 'centre of mass'-flux

$\chi \quad$ Molecular occupancy factor

$\chi \quad$ Position vector relative to particle position

$\psi \quad$ Alternative drag coefficient used by R.G. Lunnon

$\boldsymbol{\Psi} \quad$ Total momentum flux vector

$\boldsymbol{\omega} \quad$ Rate of rotation vector

$\boldsymbol{\Omega} \quad$ Total angular momentum flux vector

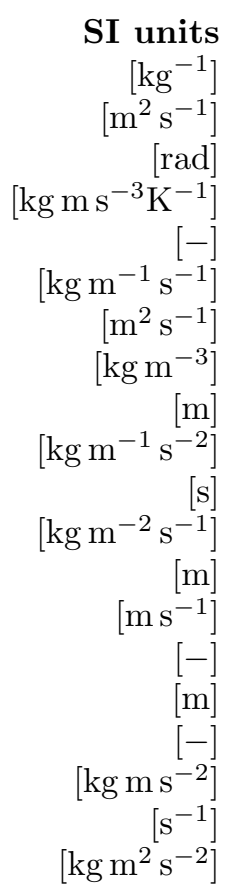

\section{Subscripts and superscripts}

\section{Symbol Description}

(0) Equilibrium 


$\begin{array}{ll}\text { AM } & \text { Added mass force } \\ \text { B } & \text { Basset history force } \\ \text { c } & \text { Continuous phase } \\ \text { d } & \text { Diffusive scale } \\ \mathrm{D} & \text { Drag force } \\ D & \text { Diameter based } \\ \mathrm{e} & \text { Due to intermolecular interaction } \\ \mathrm{H} & \text { History force } \\ \mathrm{L}, \mathrm{S} & \text { Saffman lift force } \\ \mathrm{L}, \mathrm{M} & \text { Magnus lift force } \\ \mathrm{p} & \text { Particle phase } \\ \mathrm{r} & \text { Relative } \\ \mathrm{volume} & \text { Volumetric, or body force } \\ \mathrm{VW} & \text { Van der Waals force } \\ z & \text { Vector component in axial direction } \\ \theta & \text { Vector component in azimuthal direction } \\ \kappa & \text { Heat conductive } \\ \nu & \text { Viscous } \\ \nabla \sigma & \text { Force due to gradients in stress }\end{array}$

\section{Above/Below}

\section{Symbol Description}

$\bar{a} \quad$ Average

$\widehat{a} \quad$ Special, non-dimensional case of a variable

$\widetilde{a} \quad$ Local variable in integrals

$\overline{\mathbf{a}} \quad \mathbf{a}+\mathbf{a}^{\mathrm{T}}$ 


\section{Chapter 1}

\section{INTRODUCTION}

In the oil industry, water is frequently used to maintain the pressure in an oil reservoir during the production of oil. This mechanism is one of the causes that simultaneously with oil an in time increasing quantity of water is produced. Separation of this oil-water mixture is required to recover the desired oil. Moreover, to be able to return produced water to the well, or dispose it to the environment, polluting components of the mixture have to be separated to the extent that the efflux complies with certain specifications.

Separation of phases is achieved in a series of separation stages. The first stage is bulk separation, in which a mixture of water, gas, oil and sand is roughly separated in their phases. Secondary separation is required for the removal of the undesired pollution of water-in-oil or oil-in-water from the bulk of oil or water, respectively. Produced water treatment is the removal of the oil-in-water from the bulk of water. This research focuses on produced water treatment.

Conventional separation methods, such as gravity settling, fail to remove small quantities of tiny oil-droplets from the produced water. Therefore, enhanced separation methods, such as flotation, ultra-filtration or swirling flow separation, are required. During produced water treatment the oil-water mixture can be considered a dilute multiphase fluid. For such fluids it is appropriate, without too much computational effort, to apply Lagrangian particle tracking to predict the oil-droplet trajectories that determine the distribution of the dispersed phase.

In Lagrangian particle tracking, the force on the particle is approximated in terms of forces representing the effect of specific flow phenomena, such as the drag force, the lift force, the stress gradient force, the added mass force 
and the history force. Evaluation of the history force involves integration in time, which is computationally expensive. Therefore, the history force is often neglected a priori, without considering its influence on the resulting prediction of particle motion. However, especially for relatively heavy particles, the history force cannot be neglected a priori. Therefore, the influence of the history force on the prediction of the motion of oil-droplets in time will be determined for oil-droplets, typically observed in produced water treatment, in an analytic swirling flow field typical for swirling flow separators. Also, a lattice Boltzmann method is developed in order to obtain insight in the flow about a sphere during unsteady particle motion as well as in the force on a sphere due to unsteady particle motion.

Collision and coalescence of droplets in separators, as well as collision and coalesce of bubbles and droplets in flotation devices are important and in the case of flotation essential for the performance of the separator device. Moreover, assumption of one-way coupling in particle tracking fails if collision probability is high. Therefore, a collision detection mechanism will be developed to investigate collisions in the considered flow field.

First, a short introduction in enhanced oil-water separation methods is provided. Subsequently the objectives of this research will be summarized and an outline of the thesis is presented.

\subsection{Introduction to oil-water separation methods}

\subsubsection{Swirling flow separation}

In conventional settling, the hydrostatic pressure gradient, due to gravity, is the driving force for separation of phases. Separation of phases can be enhanced by increasing the driving force. In the flow field of a swirling flow separator the hydrodynamic pressure gradient becomes the driving force. The swirl drives the oil to the centre of the device, where it can be extracted. Figure 1.1 shows such a device. In the presented device, swirl is generated by tangentially injecting the contaminated fluid. Another common method to generate swirl is to introduce an internal vaned body [37]. The oil can either be extracted at the water-outlet, in a so called co-axial swirling flow separator, or with a vortex finder at the inlet, in a so called counter-axial swirling flow separator. All methods lead to similar large scale flow behaviour. In a swirling flow separator the flow field typically consists of a combination of the velocity due to a solid body rotation and that of an inviscid vortex, a so-called Lamb-Oseen vortex, as presented in 

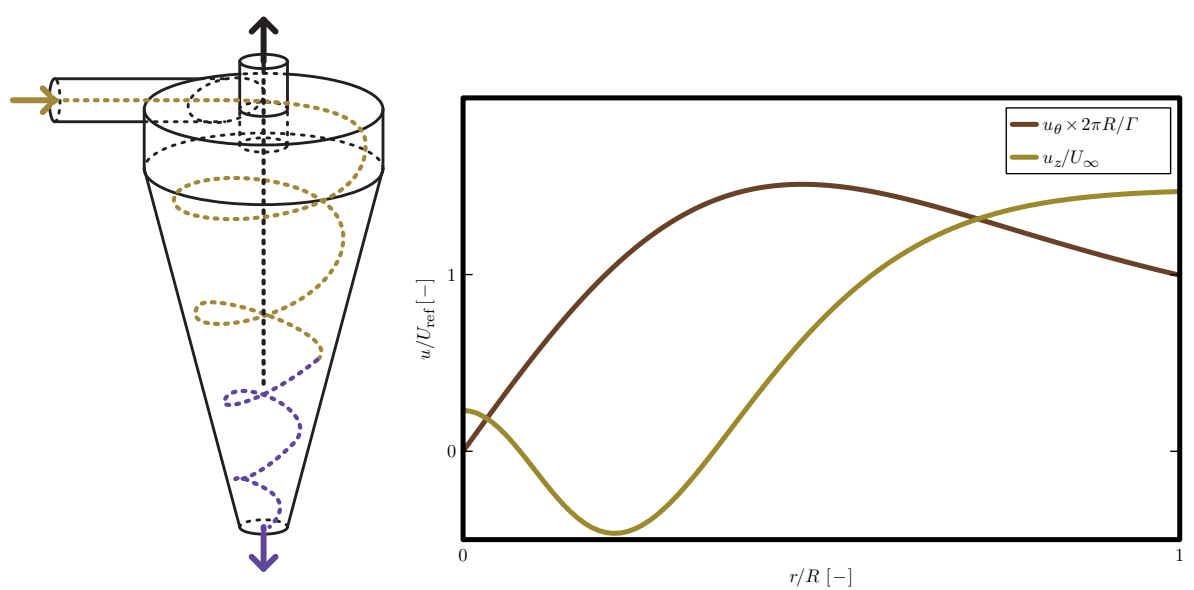

Figure 1.1: A schematic example of a tangential swirling flow separator (left) and a sketch of the axial $u_{z}$ and azimuthal $u_{\theta}$ components of the velocity field as a function of the radius typically observed $[37,20]$ in a swirling flow separator (right). The oil-water mixture enters the separator tangentially at the top, the generated swirl forces the oil to the centre of the separator. Oil is extracted with the vortex finder on the top, while treated water exits on the bottom side of the separator.

figure 1.1. Moreover, the axial velocity field typically is a W-shaped velocity profile [37, 20], as presented in figure 1.1.

\subsubsection{Bubble enhanced separation methods}

Separation of phases with conventional methods as well as with swirling flow separation is based on the density difference of the phases. However, for microscopic particles and droplets, the density difference is not sufficient to overcome drag forces. To enhance the separation methods, bubbles have been used to form agglomerates which transport contaminations at an increased rate. Bubbles can be generated in several ways $[11,33]$

- Injection of air through nozzles.

- Introduction of air through a porous surface, such as foam separation. 
- Open contact with air combined with mechanical agitation, i.e. induced air flotation (IAF) or impeller flotation.

- Dissolved air forming bubbles after a pressure drop, i.e. dissolved air flotation (DAF).

- Air is dissolved in a pressure chamber before being released to operating pressure, i.e. pressure flotation.

- Naturally dissolved air is released due to low pressure, i.e. vacuum flotation.

- Electrolysis of water resulting in oxygen and hydrogen gas, i.e. electrical flotation.

- Chemical reactions resulting in gas, i.e. chemical flotation.

Examples of methods that generate bubbles with a combination of above techniques are amongst others [22]

- Microcel. In a Microcel bubbles are generated by passing a mixture of liquid and air through an in-line static mixer. Bubble size depends on shear rate, and concentration of frother.

- Flotaire. In a Flotaire bubbles are generated by passing a liquid through a porous tube at high speed, entraining air bubbles that develop at the tube surface.

- Imox. In an Imox bubbles are generated by injecting gas at the throat of a venturi tube through which liquid flows.

Induced air flotation usually leads to relatively large bubbles in the same range as early flotation units $(2-5[\mathrm{~mm}])$ [11], which in the oil-industry are deemed too large for efficient flotation purposes. However, devices have been developed in which bubbles remain smaller due to flow phenomena. For example, in air sparged hydrocyclones (ASH's), which are in principle induced air flotation units, bubbles remain small due to large shear at the walls. For flotation tanks, dissolved air flotation is much more in use, as bubbles are in the micrometer range. A disadvantage of dissolved air flotation is that the bubble volume is limited, increasing the required size of the flotation units. Moreover, small bubbles show much slower settling rates than large bubbles, which also increases the required size of the apparatus. 


\begin{tabular}{lrr} 
Flotation technique & $\begin{array}{c}\text { Average bubble } \\
\text { diameter }[\mathrm{m}]\end{array}$ \\
\hline Electro flotation (EF) & $15 \times 10^{-6}$ & {$[12]$} \\
Gas energy management flotation (GEM) & $15-40 \times 10^{-6}$ & {$[12]$} \\
Dissolved-air flotation (DAF) & $20-50 \times 10^{-6}$ & {$[12]$} \\
Cavitation air flotation (CAF) & $30-200 \times 10^{-6}$ & {$[12]$} \\
Flocculation flotation (FF) & $100 \times 10^{-6}$ & {$[12]$} \\
Air sparged hydrocyclone flotation (ASH) & $80-200 \times 10^{-6}$ & {$[12]$} \\
Jet flotation (Jameson cell) & $300-600 \times 10^{-6}$ & {$[12]$} \\
Induced-air flotation (IAF) & $1 \times 10^{-3}$ & {$[12]$} \\
Pressure flotation & $0.5-1.5 \times 10^{-3}$ & {$[33]$} \\
Impeller flotation & $0.3-5 \times 10^{-3}$ & {$[33]$}
\end{tabular}

Table 1.1: Average bubble size reported in literature [12, 33], ordered with respect to bubble size

A novel method to include flocculation and bubble mixing is to use a hydrocyclone for mixing of air and floc generation, subsequently followed by a more conventional settling of the generated flocs [11]. This is claimed to result in good settling, as phases are already pre-separated in the hydrocylone, and a bulk-like separation can take place afterwards.

\subsubsection{Lamellar settling and flotation}

Lamellar settling is related to conventional settling. However, inclined plates or pipes are used instead of a large vessel. This results in a smaller set-up. For Acid Mine Drainage, flocculation enhanced lamellar settling requires less chemicals and less energy than flocculation enhanced dissolved air flotation [36]. However, for difficult to settle particles, lamellar settling is limited, and dissolved air flotation is required. Water treatment in oil-industry might be in the range, for which lamellar settling is of limited utility.

\subsubsection{Efficient formation of flocs}

Formation of flocs for flocculation processes involves mixing of flocculant to provide interaction and growing of flocs. One way of mixing is mechanical agitation, while another way is using plug flow to obtain a mixing flow profile [7]. The plug flow profile is usually obtained in a straight pipe. However, using 
a coiled pipe system has much advantages with respect to both mechanical agitation and straight pipes [7]. Floc settling rate is faster, while required energy is lower and the size of the device is much smaller. Important characteristic numbers that are involved in the mixing process are Reynolds number, Camp number, Dean number and Germano number. The Dean and Germano number are characteristics of the coiled tubular system, and are important for secondary flow phenomena that improve the mixing of flocculant.

Existence of air bubbles during generation of flocs is beneficial for a multitude of flotation methods [11], as generated flocs then already contain bubbles and settle very efficiently. Methods to obtain floc formation in the presence of bubbles often involve centrifugal, or cyclonic devices with an air core. The cyclonic devices show good flocculation with minor breakup. Also, cyclonic devices have been reported to efficiently uncoil flocs produced with very heavy molecular weight cationic polymers combined with anionic polyacylamide flocculants for dual polymer flocculation.

\subsubsection{Surface chemistry important for flotation}

From experimental results in $[1,2]$, it can be concluded that for high efficiency for dissolved air flotation chemical pre-treatment is an essential requirement. Addition of aluminium sulphate results in a large boost of flotation efficiency, while other parameters such as air to oil ratio, saturation pressure and recycled water ratio does not have a major impact [2]. Moreover, addition of aluminium sulphate leads to an optimal efficiency for a mixture with a $\mathrm{pH}$ of 8 with a concentration of $0.1\left[\mathrm{~kg} \mathrm{~m}^{-3}\right]$. Ferric sulphate gives higher efficiency [1] with only $0.02\left[\mathrm{~kg} \mathrm{~m}^{-3}\right]$ and at at $\mathrm{pH}$ of about 7 . The range of $\mathrm{pH}$ in which optimal performance can be achieved is wider than for aluminium sulphate. This range of $\mathrm{pH}$ agrees with the reported practice in separation processes, which is to alter the $\mathrm{pH}$ of the mixture to obtain a $\mathrm{pH}$ neutral solution [11].

High performance of addition of aluminium or ferric sulphate can be partly explained by a reduction in magnitude of the negative $\zeta$-potential. However, the $\zeta$-potential fails to explain the higher performance of ferric sulphate, indicating that DLVO forces (Derjaguin, Landau, Verwey and Overbeek) are not the only forces of importance [1]. Moreover, addition of poly-electrolytes does not result in any improvement in flotation efficiency, while the magnitude of negative $\zeta$ potential is reduced to zero for very low concentrations and turned to positive for slightly higher concentrations [2]. This is also observed in experimental results that indicate that the addition of cationic polymers to reduce the magnitude of the surface charge does not have a positive effect on flotation efficiency [11]. 
It can therefore be concluded that reducing the magnitude of the $\zeta$-potential can be very advantageous, but only if other surface chemistry, that might reduce performance, is not promoted too much.

\subsubsection{De-gassing reduces coalescence}

Coalescence is promoted in order to improve separation processes. However, for some applications a stable dispersion is required. Stable dispersions can be obtained by adding surfactants, but can also be obtained by de-gassing water [23]. De-gassing prevents cavitation to occur during coalescence, which increases the required energy to coalesce. Hydroxyl adsorption probably stabilizes the dispersion and reintroducing gas does not result in coalescence afterward. It can therefore be concluded that, in order to reduce the level of dispersion, degassing should be avoided in separation processes.

\subsection{Objectives}

Separation efficiency is influenced by both flow phenomena and chemical processes. In this research the focus is on flow phenomena in hydrocyclones:

- Assess the influence of the Basset history force for the prediction of particle trajectories in swirling flow fields;

- Investigate the history force and improve its prediction, if this force is shown to be significant;

- Obtain an efficient prediction of the history force;

- Assess the influence of coalescence outside the core of a swirling flow separator on separation efficiency;

\subsection{Outline}

\section{Chapter 2}

In chapter 2 the differential equations for the velocity, position and rate of rotation of a particle are derived and presented. 


\section{Chapter 3}

The equations of motion are the basis of Lagrangian particle tracking. The contributions to the force on a particle can be distinguished in a number of separate forces. The forces involved in the Lagrangian method are introduced in this chapter. Several correlations for the drag coefficient, i.e. the dimensionless drag force, are presented and a correlation is chosen to use in this research. Subsequently, the history force is introduced in more detail. For the history force, the Basset kernel with the assumption of Stokes' flow is shown. A more general kernel, valid in the presence of inertia, is proposed based on a kernel proposed by Mei et al.[31, 30, 27]

\section{Chapter 4}

The equations of motion, introduced in chapter 2 with the expressions for the separate forces introduced in chapter 3 are discretized in order to obtain a numerical scheme for the prediction of particle motion. First numerical treatment of the differential equation is presented. Subsequently, numerical treatment of the integral in the history force in its general formulation is presented. Lastly, numerical treatment the special case of the Basset kernel is derived from the integral in its general formulation.

\section{Chapter 5}

The discretized equations of motion are applied to the case of settling spheres. An expression for the kernel in the integral in the history force is obtained, by matching the predicted particle trajectories and experimental data obtained by Mordant \& Pinton (2000) [32].

\section{Chapter 6}

After obtaining a kernel for the integral in the history force, the case of an oil droplet injected in a swirling flow is considered. First, an analytic flow field, mimicking typically observed flows in swirling flow separators, is described. Subsequently, the discretized equations of motion are applied to an oil-droplet in that swirling flow. The equations of motion using several approximations for drag and history force are compared to each other to show the influence of these approximations on the trajectories. 


\section{Chapter 7}

Collision detection is introduced in order to obtain information about the probability of coalescence and the validity of the approach to assume that the mixture is a dilute dispersion. An efficient collision detection scheme with a work-load of $\mathcal{O}\left(N_{\mathrm{p}} \ln N_{\mathrm{p}}\right)$ is proposed. The proposed scheme is first investigated for the case of multiple settling spheres and these results are compared to results of an $\mathcal{O}\left(N_{\mathrm{p}}^{2}\right)$ scheme. Subsequently, in order to show its performance the scheme is applied for the case of oil-droplets in a swirling flow.

\section{Chapter 8}

A Lattice Boltzmann Method (LBM) for water is introduced in order to obtain the flow about a sphere. Two methods are described that are capable to take into account the equation of state of water in the LBM. Moreover, an approach to obtain macroscopic quantities is proposed, based on a Gauß-Hermite quadrature at the required peculiar velocity for such a quadrature. Bounce-back-like boundary conditions and non-reflecting in and outflow conditions are presented, exploiting the used quadrature approach. The schemes are applied to the case of a sphere in impulsively started motion to show differences in both proposed methods and to show the differences of the results of the proposed method with the results of ideal gas approaches with and without adaption of the speed of sound for such an ideal gas.

\section{Chapter 9}

Finally, concluding remarks of this research are presented, and recommendations are given for further research directions. 
Chapter 1. Introduction 


\section{CHAPTER 2}

\section{PARTICLE MOTION}

Bubbles, droplets and solid particles move in a fluid due to the force exerted by the surrounding fluid on the surface of the particle as well as due to volumetric forces. A general equation of motion can be derived, valid for bubbles, droplets and solids. The flow field about the object is required in order to obtain the force exerted by the fluid on the surface of the particle. However, computing the flow field about all particles becomes demanding for an increasing number of particles. Moreover, if the scale of the flow field in which the particle is moving is large with respect to the scale of the flow field about the object, the required resolution would make straightforward simulation not feasible. Therefore, approximations are required to capture the general flow field as well as the particle motion.

In this chapter, the general equation of motion is derived for a particle in an arbitrary flow field under influence of volumetric forces including mass transfer. And particle quantities such as position and velocity are defined.

\subsection{Equations of motion}

The motion of a particle of any nature, whether it is a bubble, a droplet or a solid, is governed by the forces acting on the particle. Conservation of momentum implies that the momentum contained by a particle changes due to forces acting on the particle and a change of momentum due to mass transfer. The equations of motion in many different formulations can be found in literature, [15]. In order to define the specific formulation, the equations of motion and its 
quantities are derived and defined. In this chapter, first the differential equation for the velocity of a particle is derived. Subsequently, the differential equations for particle position and rate of rotation are presented. Finally the system of differential equations governing the motion of a particle is presented.

\subsubsection{Linear momentum and position}

The total momentum of a particle is equal to the mass $m$ of the particle times the particle velocity $\boldsymbol{v}$. Therefore, the particle velocity is defined as

$$
\boldsymbol{v} \equiv \frac{1}{m} \iiint_{V_{\mathrm{p}}} \rho \boldsymbol{u} \mathrm{d} V
$$

with $\boldsymbol{u}$ the velocity and $\rho$ the density at that point inside the particle and $V_{\mathrm{p}}$ the volume of the particle. The mass of the particle is defined as

$$
m \equiv \iiint_{V_{\mathrm{p}}} \rho \mathrm{d} V
$$

The time rate of change of momentum contained by the particle is

$$
\frac{\mathrm{d}}{\mathrm{d} t} \iiint_{V_{\mathrm{p}}} \rho \boldsymbol{u} \mathrm{d} V=\iint_{\partial V_{\mathrm{p}}} \boldsymbol{\sigma} \cdot \boldsymbol{n} \mathrm{d} S+\iiint_{V_{\mathrm{p}}} \rho \boldsymbol{f} \mathrm{d} V+\iint_{\partial V_{\mathrm{p}}} \widetilde{\rho} \boldsymbol{u}\left(\boldsymbol{u}_{\partial V_{\mathrm{p}}}-\boldsymbol{u}\right) \cdot \boldsymbol{n} \mathrm{d} S,
$$

with $\boldsymbol{u}_{\partial V_{\mathrm{p}}}$ the local velocity of the surface of the particle, $\boldsymbol{n}$ the outward unit normal vector to the surface of the particle, $\boldsymbol{\sigma}$ the stress tensor of the stress of the surrounding fluid at the surface of the particle, $f$ the volumetric forces in the particle and $\widetilde{\rho}$ the density of the mass added to, or removed from the particle, defined as

$$
\widetilde{\rho} \equiv \begin{cases}\rho_{\text {added }} & \text { if }\left(\boldsymbol{u}_{\partial V_{\mathrm{p}}}-\boldsymbol{u}\right) \cdot \boldsymbol{n}>0 \\ \rho_{\partial V_{\mathrm{p}}} & \text { if }\left(\boldsymbol{u}_{\partial V_{\mathrm{p}}}-\boldsymbol{u}\right) \cdot \boldsymbol{n}=0 \\ \rho_{\text {removed }} & \text { if }\left(\boldsymbol{u}_{\partial V_{\mathrm{p}}}-\boldsymbol{u}\right) \cdot \boldsymbol{n}<0\end{cases}
$$

The mass flux $\phi$ through the surface, for instance due to a phase change or a chemical reaction, is defined as

$$
\phi \equiv \widetilde{\rho}\left(\boldsymbol{u}_{\partial V_{\mathrm{p}}}-\boldsymbol{u}\right) \cdot \boldsymbol{n} .
$$




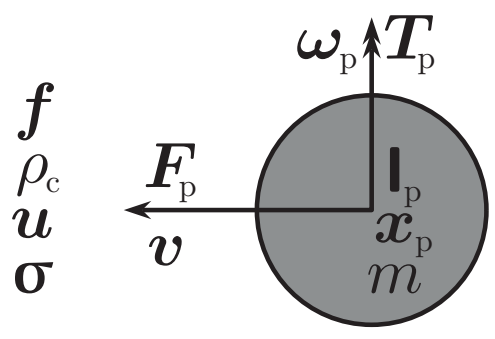

Figure 2.1: A particle in a flow field subjected to volumetric forces and the stress of the flow field exerted at the surface of the particle.

Therefore, the total change in momentum due to the mass flux $\boldsymbol{\Psi}$ becomes

$$
\boldsymbol{\Psi}=\iint_{\partial V_{\mathrm{p}}} \phi \boldsymbol{u} \mathrm{d} S .
$$

Stress $\boldsymbol{\sigma}$ acting on the surface of the particle and volumetric force $\boldsymbol{f}$ contribute to the force acting on the particle $\boldsymbol{F}_{\mathrm{p}}$, which is defined as

$$
\boldsymbol{F}_{\mathrm{p}} \equiv \iint_{\partial V_{\mathrm{p}}} \boldsymbol{\sigma} \cdot \boldsymbol{n} \mathrm{d} S+\iiint_{V_{\mathrm{p}}} \rho \boldsymbol{f} \mathrm{d} V .
$$

Often the volumetric force per unit mass is the gravitational acceleration $\boldsymbol{g}$. The stress, resulting from the motion of the fluid around the particle, consists of pressure and viscous stresses. The volumetric and the surface contribution to the force on the particle, as well as the important variables of the flow field, are shown in figure 2.1.

Using the definition of the force on the particle, equation (2.7), and the change of momentum due to a possible mass flux, equation (2.6), the equation for the change of momentum, equation (2.3), becomes

$$
\frac{\mathrm{d}}{\mathrm{d} t}(m \boldsymbol{v})=\boldsymbol{F}_{\mathrm{p}}+\boldsymbol{\Psi} .
$$

The velocity of the particle and the mass of the particle are now defined. However, the particle location is not yet defined. In this research the particle 
location $\boldsymbol{x}_{\mathrm{p}}$ is defined as the centre of mass of the particle, i.e.

$$
\boldsymbol{x}_{\mathrm{p}} \equiv \frac{1}{m} \iiint_{V_{\mathrm{p}}} \rho \boldsymbol{x} \mathrm{d} V .
$$

Therefore, the time rate of change of the particle location is governed by the velocity of the particle as well as the mass flux at the surface of the particle. Derivation of the differential equation for the particle location is provided in appendix A.1. The differential equation for the particle location is

$$
\frac{\mathrm{d} \boldsymbol{x}_{\mathrm{p}}}{\mathrm{d} t}=\boldsymbol{v}+\boldsymbol{\Phi}
$$

where $\boldsymbol{\Phi}$ is the total mass flux with respect to the centre of mass defined as

$$
\boldsymbol{\Phi} \equiv \frac{1}{m} \iint_{\partial V_{\mathrm{p}}}\left(\boldsymbol{x}-\boldsymbol{x}_{\mathrm{p}}\right) \phi \mathrm{d} S .
$$

\subsubsection{Angular momentum}

A particle does not only have a translational motion, but also a rotational motion. The angular momentum of a particle is defined as

$$
\mathbf{I} \cdot \boldsymbol{\omega} \equiv \iiint_{V_{\mathrm{p}}} \boldsymbol{x} \times \rho \boldsymbol{u} \mathrm{d} V,
$$

with $\boldsymbol{\omega}$ the rate of rotation and $\mathbf{I}$ the moment of inertia of the particle, evaluated for a fixed reference frame of the particle,

$$
\mathbf{I} \equiv \iiint_{V_{\mathrm{p}}} \rho\left(\|\boldsymbol{x}\|^{2} \mathbf{U}-\boldsymbol{x} \boldsymbol{x}\right) \mathrm{d} V,
$$

with $\mathbf{U}$ the unit, or identity, matrix

$$
\mathbf{U}=\left[\begin{array}{lll}
1 & 0 & 0 \\
0 & 1 & 0 \\
0 & 0 & 1
\end{array}\right]
$$


The time rate of change of angular momentum $\mathbf{I} \cdot \boldsymbol{\omega}$ with respect to a fixed reference frame of the particle is caused by the torque $\boldsymbol{T}$ and is expressed, similar to equations (2.8) and (2.7), as

$$
\frac{\mathrm{d}}{\mathrm{d} t}(\mathbf{I} \cdot \boldsymbol{\omega})=\boldsymbol{T}+\iint_{\partial V_{\mathrm{p}}} \phi \boldsymbol{x} \times \boldsymbol{u} \mathrm{d} S .
$$

Volumetric forces and surface stresses contribute to the the torque $\boldsymbol{T}$, which becomes

$$
\boldsymbol{T} \equiv \iint_{\partial V_{\mathrm{p}}} \boldsymbol{x} \times(\boldsymbol{\sigma} \cdot \boldsymbol{n}) \mathrm{d} S+\iiint_{V_{\mathrm{p}}} \rho \boldsymbol{x} \times \boldsymbol{f} \mathrm{d} V .
$$

The rate of rotation of a particle with respect to its centre of mass is of more use for local phenomena than the rate of rotation with respect to a fixed reference frame. The angular momentum of the particle with respect to the centre of mass of the particle, i.e. $\mathbf{I}_{\mathrm{p}} \cdot \boldsymbol{\omega}_{\mathrm{p}}$, is related to the torque on the particle with respect to the centre of mass as well as the effect of the flux of angular momentum $\Omega$ with respect to the centre of mass. Derivation of the equation of conservation of angular momentum with respect to the centre of mass is provided in appendix A.2. It results in

$$
\frac{\mathrm{d}}{\mathrm{d} t}\left(\mathbf{I}_{\mathrm{p}} \cdot \boldsymbol{\omega}_{\mathrm{p}}\right)=\boldsymbol{T}_{\mathrm{p}}+\boldsymbol{\Omega}
$$

with $\mathbf{I}_{\mathrm{p}}$ the moment of inertia of the particle, evaluated at the centre of mass $\boldsymbol{x}_{\mathrm{p}}$ of the particle

$$
\mathbf{I}_{\mathrm{p}} \equiv \iiint_{V_{\mathrm{p}}} \rho\left(\|\boldsymbol{\chi}\|^{2} \mathbf{U}-\boldsymbol{\chi} \boldsymbol{\chi}\right) d V
$$

with $\chi$ the location relative to the centre of mass

$$
\chi \equiv \boldsymbol{x}-\boldsymbol{x}_{\mathrm{p}}
$$

For a spherical particle with constant density, the moment of inertia is a scalar times the unit matrix as shown in section B.6.1. Therefore, the inner product of the moment of inertia tensor and the rate of rotation, $\mathbf{I}_{\mathrm{p}} \cdot \boldsymbol{\omega}_{\mathrm{p}}$, can be replaced by the product of the scalar moment of inertia and the rate of rotation, i.e. $I_{\mathrm{p}} \boldsymbol{\omega}_{\mathrm{p}}$. 
The torque on the particle with respect to its centre of mass $\boldsymbol{T}_{\mathrm{p}}$ is

$$
\boldsymbol{T}_{\mathrm{p}} \equiv \iint_{\partial V_{\mathrm{p}}} \boldsymbol{\chi} \times(\boldsymbol{\sigma} \cdot \boldsymbol{n}) \mathrm{d} S+\iiint_{V_{\mathrm{p}}} \rho \boldsymbol{\chi} \times \boldsymbol{f} \mathrm{d} V .
$$

The contribution of the volume integral is equal to zero in a constant volumetric force field $\boldsymbol{f}$, as the particle location $\boldsymbol{x}_{\mathrm{p}}$ is defined to coincide with its centre of mass.

Finally, the influence of mass flux $\Omega$ is

$$
\boldsymbol{\Omega} \equiv \iint_{\partial V_{\mathrm{p}}} \phi \boldsymbol{\chi} \times \boldsymbol{u} \mathrm{d} S+m \boldsymbol{v} \times \boldsymbol{\Phi} .
$$

\subsection{System of differential equations}

The differential equations governing particle location (2.10), velocity (2.8) and rate of rotation (2.17) have been derived in this chapter, leading to a system of coupled first order ordinary differential equations.

$$
\frac{\mathrm{d}}{\mathrm{d} t}\left(\begin{array}{c}
\boldsymbol{x}_{\mathrm{p}} \\
m \boldsymbol{v} \\
\mathbf{I}_{\mathrm{p}} \cdot \boldsymbol{\omega}_{\mathrm{p}}
\end{array}\right)=\left(\begin{array}{c}
\boldsymbol{v}+\boldsymbol{\Phi} \\
\boldsymbol{F}_{\mathrm{p}}+\boldsymbol{\Psi} \\
\boldsymbol{T}_{\mathrm{p}}+\boldsymbol{\Omega}
\end{array}\right) .
$$

This leaves us with the calculation of the forces and mass fluxes in order to close the system of differential equations. Moreover, for non-spherical particles the orientation of the particle is also of importance. This implies that a reference orientation has to be determined, for instance the orientation for which the moment of inertia is a diagonal matrix with entries ordered according to magnitude. 


\section{CHAPter 3}

\section{FORCE AND TORQUE}

In the preceding chapter, the system of differential equations for particle motion was derived. In this research an approximation of the actual force and torque on a particle lead to an equation of motion that requires the macroscopic flow variables only. In this research the motion of small oil-droplets and small contaminated bubbles is considered. In this research these small contaminated oil-droplets and small contaminated bubbles are assumed to behave as solid spherical particles in order to obtain closure relations for the description of the force and torque involved. The various contributions to the force and torque are described and the drag force and history force are presented in more detail.

\subsection{Approximation of the force and torque}

In order to obtain the force on a particle due to the surface stress, a fully resolved simulation of the in general time-dependent flow about the particle is required. For most practical applications this is not feasible. Therefore, the surface integral is approximated by a sum of contributions to the force that represent separate effects of the flow field and of the particle motion. These contributions depend on the macroscopic flow field variables and their derivatives, at the centre of mass of the particle. The effects taken into account in this research are the volumetric forces, the drag, the divergence of the stress tensor, the added mass force, the Saffmann and Magnus lift forces and the history force. 
Therefore the force on the particle is expressed as

$$
\boldsymbol{F}_{\mathrm{p}}=\boldsymbol{F}_{\text {volume }}+\boldsymbol{F}_{\boldsymbol{\nabla} \cdot \boldsymbol{\sigma}}+\boldsymbol{F}_{\mathrm{AM}}+\boldsymbol{F}_{\mathrm{L}, \mathrm{S}}+\boldsymbol{F}_{\mathrm{L}, \mathrm{M}}+\boldsymbol{F}_{\mathrm{D}}+\boldsymbol{F}_{\mathrm{H}}+\boldsymbol{F}_{\text {Not captured }},
$$

where $\boldsymbol{F}_{\text {Not captured }}$ represents the effects that are not, or not fully, represented by the various contributions to the force considered.

The contribution of the volumetric force is the approximation of the volume integral in equation (2.7). Usually this integral can be evaluated quite straightforward, leading to an exact expression of the volumetric force. The other contributions to the force are an approximation of the surface integral in equation (2.7). The force computed using the flow variables taken at the centre of mass of the particle, can be corrected using the Faxén force correction. Taking into account the Laplacian of both the variables and its derivatives of the flow field is sufficient to capture both first and second order effects for a spherical particle.

Similarly, the torque exerted on a particle by the flow is

$$
\boldsymbol{T}_{\mathrm{p}}=\boldsymbol{T}_{\text {volume }}+\boldsymbol{T}_{\boldsymbol{\nabla} \cdot \boldsymbol{\sigma}}+\boldsymbol{T}_{\boldsymbol{\omega}_{\text {relative }}}+\boldsymbol{T}_{\text {Faxén }}+\boldsymbol{T}_{\text {Not captured }} .
$$

In this research, contributions from the added mass force, the drag force, the history force and the lift forces to the torque are considered Faxén torque effects.

Crowe et al. (1998) [15] and Loth \& Dorgan (2009) [28] provide an overview of the forces involved $[17,18,19]$. In this chapter some of the forces are derived exactly, while for others an approximation using expressions involving coefficients is provided.

\subsection{Dimensionless and characteristic numbers}

The particle and the flow about the particle can be characterized by dimensionless numbers and characteristic scales. These dimensionless numbers and characteristic scales are used in the approximation of various contributions to the force. Therefore, they will be introduced first.

Characteristic time-scales $\tau$ describe the fluid motion about the particle. One time-scale is the diffusive time-scale $\tau_{\mathrm{d}}$. The diffusive time-scale determines how fast the flow reacts to changes in the relative velocity of the particle and is defined as

$$
\tau_{\mathrm{d}} \equiv \frac{\mathcal{L}^{2}}{\mathcal{N}}
$$

with characteristic length $\mathcal{L}$ and characteristic kinematic viscosity $\mathcal{N}$. For a spherical particle, the characteristic length is the diameter $D$ of the particle 
and the characteristic kinematic viscosity is the reference kinematic viscosity $\nu$ of the continuous phase. Therefore,

$$
\tau_{\mathrm{d}}=\frac{D^{2}}{\nu} .
$$

Another time-scale is the advective time-scale $\tau_{\mathrm{a}}$. The advective time-scale determines how fast the particle is moving relative to the fluid and is defined as

$$
\tau_{\mathrm{a}} \equiv \frac{\mathcal{L}}{\mathcal{U}}
$$

with characteristic velocity $\mathcal{U}$. For particle motion, the characteristic velocity $\mathcal{U}$ is the magnitude of the relative velocity of the particle with respect to the flow. The relative velocity is defined as

$$
\boldsymbol{w} \equiv \boldsymbol{v}-\boldsymbol{u}
$$

where $\boldsymbol{u}$ is the velocity of the fluid in absence of the particle and $\boldsymbol{v}$ is the particle velocity defined in equation (2.1).

The Reynolds number determines the importance of advective effects relative to the diffusive effects in the momentum equation. It is the ratio of the diffusive time-scale and the advective time-scale

$$
\operatorname{Re} \equiv \frac{\mathcal{L U}}{\mathcal{N}}
$$

In this research, for a particle with diameter $D$, velocity $\boldsymbol{w}$ and a continuous phase with viscosity $\nu$, the Reynolds number is

$$
\operatorname{Re}_{\mathrm{D}}=\frac{D\|\boldsymbol{w}\|}{\nu} .
$$

The vorticity of a flow field is defined by

$$
\boldsymbol{\omega}_{\mathrm{c}} \equiv \boldsymbol{\nabla} \times \boldsymbol{u}
$$

Moreover, the dotproduct of a vector $\boldsymbol{a}$ and the gradient of the velocity field can be written as

$$
\boldsymbol{a} \cdot \boldsymbol{\nabla u}=\boldsymbol{a} \cdot \overline{\overline{\nabla u}}-\boldsymbol{a} \times \frac{\boldsymbol{\omega}_{\mathrm{c}}}{2},
$$


where

$$
\overline{\overline{\nabla \boldsymbol{u}}} \equiv \frac{1}{2}\left[(\boldsymbol{\nabla u})^{\mathrm{T}}+\boldsymbol{\nabla u}\right],
$$

is the symmetric part of the gradient.

The spin number $\Omega$ compares rotation of the flow to the velocity of the flow

$$
\Omega \equiv \frac{\mathcal{L W}}{\mathcal{U}}
$$

with characteristic rotation $\mathcal{W}$. For a rotating spherical particle in a rotating flow $[29,15,28]$, the characteristic rotation rate is the magnitude of the relative rotation $\left\|\boldsymbol{\omega}_{\mathrm{r}}\right\|$ defined as

$$
\boldsymbol{\omega}_{\mathrm{r}} \equiv \boldsymbol{\omega}_{\mathrm{p}}-\frac{1}{2} \boldsymbol{\omega}_{\mathrm{c}} \text {. }
$$

Moreover, the characteristic length is particle diameter $D$ and the characteristic velocity is the magnitude of the relative velocity $\|\boldsymbol{w}\|$, leading to

$$
\Omega=\frac{D\left\|\boldsymbol{\omega}_{\mathrm{r}}\right\|}{\|\boldsymbol{w}\|} .
$$

Similarly a shear flow is characterized by a shear number $\alpha[29,15,28]$. The shear number relates the magnitude of the perturbation on the velocity by rotation. The magnitude of the perturbation by rotation with length $D$ is $\frac{D}{2}\left\|\boldsymbol{\omega}_{\mathrm{c}}\right\|$ and the characteristic velocity is $\|\boldsymbol{w}\|$, leading to

$$
\alpha=\frac{D\left\|\boldsymbol{\omega}_{\mathrm{c}}\right\|}{2\|\boldsymbol{w}\|} .
$$

Moreover, a rotation Reynolds number [15, 29, 28] for a rotating particle in a rotating flow is defined as

$$
\operatorname{Re}_{\omega} \equiv \frac{\mathcal{L}^{2} \mathcal{W}}{\mathcal{N}}=\Omega \operatorname{Re}
$$

For a spherical particle this becomes

$$
\operatorname{Re}_{\omega}=\frac{D^{2}\left\|\boldsymbol{\omega}_{\mathrm{r}}\right\|}{\nu}
$$


The density ratio $\widehat{\rho}$ of the particle density $\rho_{\mathrm{p}}$ and the density of the continuous phase $\rho_{\mathrm{c}}$ is an important parameter for particle motion. The density ratio is defined as

$$
\widehat{\rho} \equiv \frac{\rho_{\mathrm{p}}}{\rho_{\mathrm{c}}}
$$

\subsection{Volume force and torque}

The volume force is the evaluation or approximation of the volume integral in equation (2.7)

$$
\boldsymbol{F}_{\text {volume }} \equiv \iiint_{V_{\mathrm{p}}} \widetilde{\rho}_{\mathrm{p}} \widetilde{\boldsymbol{f}} \mathrm{d} V
$$

where $\widetilde{\boldsymbol{f}}$ is the local volumetric force, and $\widetilde{\rho}_{\mathrm{p}}$ is the local particle density.

The volumetric contribution to the torque on a particle is

$$
\boldsymbol{T}_{\text {volume }} \equiv \iiint_{V_{\mathrm{p}}} \tilde{\rho}_{\mathrm{p}} \chi \times \tilde{\boldsymbol{f}} \mathrm{d} V
$$

The volumetric force and the volumetric torque can be approximated by a Taylor series expansion with respect to the particle location $\boldsymbol{x}_{]}$. First, the result of such an approximation will be presented for an arbitrarily shaped particle. In this research, particles are assumed to be spherical particles of uniform density. This makes it possible to evaluate integrals that are a result of the Taylor series expansion. Therefore, the result for a Taylor series approximation for spherical particles of uniform density will be presented next.

\subsubsection{Particle of an arbitrary shape}

Using the definition of the mass of a particle, equation (2.2), and the definition of the centre of mass of a particle, equation (2.9), the volume force expressed in a Taylor series for an arbitrary shape becomes

$$
\boldsymbol{F}_{\text {volume }}=m \boldsymbol{f}+\frac{1}{2} \iiint_{V_{\mathrm{p}}} \widetilde{\rho}_{\mathrm{p}} \chi \chi \mathrm{d} V: \nabla \nabla \boldsymbol{f}+\iiint_{V_{\mathrm{p}}} \widetilde{\rho}_{\mathrm{p}} \sum_{i=3}^{\infty} \frac{\chi^{i}}{i !} \frac{\partial^{i} \boldsymbol{f}}{\partial \boldsymbol{x}^{i}} \mathrm{~d} V,
$$

where $\boldsymbol{f}$ and $\boldsymbol{\nabla} \nabla \boldsymbol{f}$ are evaluated at the particle centre of mass. 
A similar approach provides the torque on a particle due to volumetric forces. A Taylor series expansion of the volumetric force field leads to

$$
\begin{array}{r}
\boldsymbol{T}_{\text {volume }}=\iiint_{V_{\mathrm{p}}} \tilde{\rho}_{\mathrm{p}} \boldsymbol{\chi} \mathrm{d} V \times \boldsymbol{f}+\left(\iiint_{V_{\mathrm{p}}} \widetilde{\rho}_{\mathrm{p}} \boldsymbol{\chi} \chi \mathrm{d} V \cdot \nabla\right) \times \boldsymbol{f} \\
+\iiint_{V_{\mathrm{p}}} \widetilde{\rho}_{\mathrm{p}} \boldsymbol{\nabla} \times \sum_{i=2}^{\infty} \frac{\chi^{i}}{i !} \frac{\partial^{i} \boldsymbol{f}}{\partial \boldsymbol{x}^{i}} \mathrm{~d} V .
\end{array}
$$

The first term on the right hand side is zero due to the definition of the particle position. Therefore equation (3.22) becomes

$$
\boldsymbol{T}_{\text {volume }}=\left(\iiint_{V_{\mathrm{p}}} \widetilde{\rho}_{\mathrm{p}} \chi \chi \mathrm{d} V \cdot \nabla\right) \times \boldsymbol{f}+\iiint_{V_{\mathrm{p}}} \widetilde{\rho}_{\mathrm{p}} \chi \times \sum_{i=2}^{\infty} \frac{\chi^{i}}{i !} \frac{\partial^{i} \boldsymbol{f}}{\partial \boldsymbol{x}^{i}} \mathrm{~d} V .
$$

\subsubsection{Sphere of uniform density}

If the body of interest is a spherical object, integrals for spatial moments can be evaluated. The moments for a sphere, derived in appendix B, and presented in table B.2 are used to simplify equation (3.21). Moreover, similar to the evaluation of the moment of inertia in section B.6, for the density the ratio of the mass and the volume will be substituted. The volume force for a uniform sphere is

$$
\frac{1}{m} \boldsymbol{F}_{\text {volume }}=\boldsymbol{f}+\frac{D^{2}}{40} \nabla^{2} \boldsymbol{f}+\frac{6}{\pi D^{3}} \iiint_{V_{\mathrm{p}}} \sum_{i=2}^{\infty} \frac{\chi^{2 i}}{(2 i) !} \frac{\partial^{2 i} \boldsymbol{f}}{\partial \boldsymbol{x}^{2 i}} \mathrm{~d} V
$$

and thus

$$
\frac{1}{m} \boldsymbol{F}_{\text {volume }}=\boldsymbol{f}+\frac{D^{2}}{40} \nabla^{2} \boldsymbol{f}+\mathcal{O}\left(\frac{\mathcal{F}}{m}\left\{\frac{D}{\mathcal{L}}\right\}^{4}\right),
$$

where $\mathcal{L}$ is the characteristic length scale of the volumetric force field with characteristic force $\mathcal{F}$. For instance, the characteristic force can be defined as $\mathcal{F}=\max _{\|\boldsymbol{\chi}\|<\mathcal{L}}\left\|m \boldsymbol{f}_{\boldsymbol{\chi}}\right\|$. Note that for a sphere with non-uniform density, the expression in equation (3.25) is only accurate up to order $\mathcal{O}\left(\mathcal{F}\left\{\frac{D}{\mathcal{L}}\right\}^{2}\right)$ if derivatives 
of the density of second or higher order are non-zero, and accurate up to order $\mathcal{O}\left(\mathcal{F}\left\{\frac{D}{\mathcal{L}}\right\}^{3}\right)$ if only the gradient of the density is non-zero and all higher order derivatives are zero.

In a similar way, the torque for a sphere of uniform density is

$$
\begin{aligned}
\frac{1}{I_{\mathrm{p}}} \boldsymbol{T}_{\text {volume }}=\frac{1}{2} \boldsymbol{\nabla} \times & \left\{\boldsymbol{f}+\frac{5 D^{2}}{168} \nabla^{2} \boldsymbol{f}\right\} \\
& +\frac{60}{\pi D} \iiint_{V_{\mathrm{p}}} \chi \times \sum_{i=1}^{\infty} \frac{\chi^{2 i+1}}{(2 i+1) !} \frac{\partial^{2 i+1} \boldsymbol{f}}{\partial \boldsymbol{x}^{2 i+1}} \mathrm{~d} V
\end{aligned}
$$

where $I_{\mathrm{p}}$ is the moment of inertia for a sphere. In appendix B it is derived that $I_{\mathrm{p}}=\frac{1}{10} m D^{2}$ see equation (B.37). Therefore, the torque for a sphere of uniform density becomes

$$
\frac{1}{I_{\mathrm{p}}} \boldsymbol{T}_{\text {volume }} \approx \frac{1}{2} \boldsymbol{\nabla} \times\left\{\boldsymbol{f}+\frac{5 D^{2}}{168} \nabla^{2} \boldsymbol{f}\right\}+\mathcal{O}\left(\frac{\mathcal{T}}{I_{\mathrm{p}}}\left\{\frac{D}{\mathcal{L}}\right\}^{4}\right)
$$

where $\mathcal{T}$ is the characteristic torque of the volumetric force field. For instance, the characteristic torque can be defined as $\mathcal{T}=\max _{\|\boldsymbol{\chi}\|<\mathcal{L}}\left\|m \frac{D^{2}}{20} \boldsymbol{\nabla} \times \boldsymbol{f}_{\boldsymbol{\chi}}\right\|$.

\subsection{Stress divergence force and torque}

The stress divergence force $F_{\boldsymbol{\nabla} \cdot \boldsymbol{\sigma}}$ is often called the pressure gradient force [15]. It represents the effect of the stress $\sigma$ of the macroscopic flow field acting at the surface of the particle in absence of the particle. Therefore, the force is expressed as

$$
\boldsymbol{F}_{\boldsymbol{\nabla} \cdot \boldsymbol{\sigma}} \equiv \iint_{\partial V_{\mathrm{p}}} \tilde{\boldsymbol{\sigma}} \cdot \boldsymbol{n} \mathrm{d} S
$$

where $\widetilde{\boldsymbol{\sigma}}$ is the local stress of the macroscopic flow field.

Using the divergence theorem, the surface integral of the normal component of a vector quantity can be expressed as a volume integral of the divergence of that quantity over the volume enclosed by that surface. Therefore, the surface integral of the normal component of the stress acting at the surface can be expressed as the volume integral of the divergence of the stress. The stress 
gradient force can then be defined as

$$
\boldsymbol{F}_{\boldsymbol{\nabla} \cdot \boldsymbol{\sigma}} \equiv \iiint_{V_{\mathrm{p}}} \widetilde{\boldsymbol{\nabla} \cdot \boldsymbol{\sigma}} \mathrm{d} V
$$

In absence of viscous stresses, only the pressure term contributes to the stress, which is the origin of the name of the pressure gradient force.

For a fluid with velocity $\boldsymbol{u}$, using the Navier-Stokes equations, the divergence of the stress is substituted by the density of the continuous phase $\rho_{\mathrm{c}}$ in absence of the particle times the material derivative of the velocity $\frac{\mathrm{D} \boldsymbol{u}}{\mathrm{D} t}$ minus the density of the continuous phase in absence of the particle times the volumetric force. This results in

$$
\boldsymbol{F}_{\boldsymbol{\nabla} \cdot \boldsymbol{\sigma}} \equiv \iiint_{V_{\mathrm{p}}} \widetilde{\rho}_{\mathrm{c}}\left\{\frac{\widetilde{\mathrm{D} \boldsymbol{u}}}{\mathrm{D} t}-\widetilde{\boldsymbol{f}}\right\} \mathrm{d} V
$$

where $\frac{\widetilde{\mathrm{D} u}}{\mathrm{D} t}$ is the local material derivative of the macroscopic flow field and $\widetilde{\rho_{\mathrm{c}}}$ is the local density of the continuous phase.

In a similar way the torque exterted by the macroscopic stress on the particle is defined by

$$
\boldsymbol{T}_{\boldsymbol{\nabla} \cdot \boldsymbol{\sigma}} \equiv \iint_{\partial V_{\mathrm{p}}} \chi \times(\widetilde{\boldsymbol{\sigma}} \cdot \boldsymbol{n}) \mathrm{d} S
$$

Due to the cross product, each resulting term is treated separately in the application of the divergence theorem. This results in

$$
\boldsymbol{T}_{\boldsymbol{\nabla} \cdot \boldsymbol{\sigma}} \equiv \iiint_{V_{\mathrm{p}}}\left(\begin{array}{l}
\widetilde{\sigma}_{32}-\widetilde{\sigma}_{23} \\
\widetilde{\sigma}_{13}-\widetilde{\sigma}_{31} \\
\tilde{\sigma}_{21}-\widetilde{\sigma}_{12}
\end{array}\right)+\chi \times[\widetilde{\boldsymbol{\nabla} \cdot \boldsymbol{\sigma}}] \mathrm{d} V
$$

where $\widetilde{\sigma}_{i j}$ is the $i, j$-th component of the local stress matrix.

In case of a Newtonian fluid, the stress matrix is symmetric, resulting in

$$
\boldsymbol{T}_{\boldsymbol{\nabla} \cdot \boldsymbol{\sigma}}=\iiint_{V_{\mathrm{p}}} \chi \times[\widetilde{\nabla \cdot \sigma}] \mathrm{d} V
$$




\subsubsection{Symmetric stress matrix for an arbitrary particle}

Similar to the volumetric force a Taylor series expansion of the terms in the intrglar for the stress divergence force results in

$$
\begin{array}{r}
\boldsymbol{F}_{\boldsymbol{\nabla} \cdot \boldsymbol{\sigma}}=\frac{m}{\widehat{\rho}}\left\{\frac{\mathrm{D} \boldsymbol{u}}{\mathrm{D} t}-\boldsymbol{f}\right\}+\iiint_{V_{\mathrm{p}}} \widetilde{\rho}_{\mathrm{p}} \chi \chi \mathrm{d} V: \nabla \boldsymbol{\nabla}\left[\frac{1}{\widehat{\rho}}\left\{\frac{\mathrm{D} \boldsymbol{u}}{\mathrm{D} t}-\boldsymbol{f}\right\}\right] \\
+\iiint_{V_{\mathrm{p}}} \widetilde{\rho}_{\mathrm{p}} \sum_{i=3}^{\infty} \frac{\chi^{i}}{i !} \frac{\partial^{i}}{\partial \boldsymbol{x}^{i}}\left[\frac{1}{\widehat{\rho}}\left\{\frac{\mathrm{D} \boldsymbol{u}}{\mathrm{D} t}-\boldsymbol{f}\right\}\right] \mathrm{d} V,
\end{array}
$$

where $\hat{\rho}$ is the density ratio of the particle density and the density of the continuous phase as defined in (3.18).

A Taylor series expansion for the torque results in

$$
\begin{array}{r}
\boldsymbol{T}_{\boldsymbol{\nabla} \cdot \boldsymbol{\sigma}}=\left(\iiint_{V_{\mathrm{p}}} \widetilde{\rho}_{\mathrm{p}} \boldsymbol{\chi} \boldsymbol{\chi} \mathrm{d} V \cdot \boldsymbol{\nabla}\right) \times\left[\frac{1}{\widehat{\rho}}\left\{\frac{\mathrm{D} \boldsymbol{u}}{\mathrm{D} t}-\boldsymbol{f}\right\}\right] \\
+\iiint_{V_{\mathrm{p}}} \widetilde{\rho}_{\mathrm{p}} \boldsymbol{\chi} \times \sum_{i=2}^{\infty} \frac{\chi^{i}}{i !} \frac{\partial^{i}}{\partial \boldsymbol{x}^{i}}\left[\frac{1}{\widehat{\rho}}\left\{\frac{\mathrm{D} \boldsymbol{u}}{\mathrm{D} t}-\boldsymbol{f}\right\}\right] \mathrm{d} V .
\end{array}
$$

\subsubsection{Symmetric stress matrix for a sphere of uniform density}

For a uniform sphere the stress divergence force, equation (3.34), can be reduced to

$$
\frac{\boldsymbol{F}_{\boldsymbol{\nabla} \cdot \boldsymbol{\sigma}}}{m}=\frac{1}{\widehat{\rho}}\left\{\frac{\mathrm{D} \boldsymbol{u}}{\mathrm{D} t}-\boldsymbol{f}\right\}+\frac{D^{2}}{40} \nabla^{2}\left[\frac{1}{\widehat{\rho}}\left\{\frac{\mathrm{D} \boldsymbol{u}}{\mathrm{D} t}-\boldsymbol{f}\right\}\right]+\mathcal{O}\left(\frac{\mathcal{F}}{m}\left\{\frac{D}{\mathcal{L}}\right\}^{4}\right) .
$$

Similarly the stress divergence torque, equation (3.35), can be reduced to

$$
\begin{array}{r}
\frac{\boldsymbol{T}_{\boldsymbol{\nabla} \cdot \boldsymbol{\sigma}}}{I_{p}}=\frac{1}{2} \boldsymbol{\nabla} \times\left[\frac{1}{\hat{\rho}}\left\{\frac{\mathrm{D} \boldsymbol{u}}{\mathrm{D} t}-\boldsymbol{f}\right\}+\frac{5 D^{2}}{168} \nabla^{2}\left(\frac{1}{\widehat{\rho}}\left\{\frac{\mathrm{D} \boldsymbol{u}}{\mathrm{D} t}-\boldsymbol{f}\right\}\right)\right] \\
+\mathcal{O}\left(\frac{\mathcal{T}}{I_{p}}\left\{\frac{D}{\mathcal{L}}\right\}^{4}\right) .
\end{array}
$$


The curl of the material derivative can be written in terms of vorticity $\boldsymbol{\omega}_{\mathrm{c}}$ as

$$
\boldsymbol{\nabla} \times \frac{\mathrm{D} \boldsymbol{u}}{\mathrm{D} t} \equiv \frac{\mathrm{D} \boldsymbol{\omega}_{\mathrm{c}}}{\mathrm{D} t}+\boldsymbol{\omega}_{\mathrm{c}} \boldsymbol{\nabla} \cdot \boldsymbol{u}-\boldsymbol{\omega}_{\mathrm{c}} \cdot \boldsymbol{\nabla} \boldsymbol{u}
$$

\subsubsection{Buoyancy force}

Often the volume force and the hydrostatic part of the stress divergence force are combined and called the buoyancy force, while the hydrodynamic part of the stress divergence force is then sometimes called the pressure gradient force. This means that

$$
\boldsymbol{F}_{\text {volume }}+\boldsymbol{F}_{\boldsymbol{\nabla} \cdot \boldsymbol{\sigma}}=\boldsymbol{F}_{\text {buoyancy }}+\boldsymbol{F}_{\boldsymbol{\nabla} \cdot \boldsymbol{\sigma}, \mathrm{dyn}} .
$$

In this way the buoyancy force is

$$
\boldsymbol{F}_{\text {buoyancy }} \equiv \iiint_{V_{\mathrm{p}}} \widetilde{\rho}_{\mathrm{p}}\left\{\widetilde{\boldsymbol{f}}-\frac{1}{\widetilde{\widehat{\rho}}} \widetilde{\boldsymbol{f}}\right\} \mathrm{d} V,
$$

and the hydrodynamic part of the stress divergence force is

$$
\boldsymbol{F}_{\boldsymbol{\nabla} \cdot \boldsymbol{\sigma}, \mathrm{dyn}} \equiv \iiint_{V_{\mathrm{p}}} \widetilde{\rho}_{\mathrm{c}} \frac{\widetilde{\mathrm{D} \boldsymbol{u}}}{\mathrm{D} t} \mathrm{~d} V
$$

\subsection{Added mass force}

Acceleration of the particle with respect to the large scale fluid motion will cause acceleration of the continuous phase as well. This causes the particle to behave as if it is heavier than its actual mass. Therefore, the force representing this effect is called, the added, or virtual, mass force. The added mass force is an effect based on the volume of the particle. Therefore, the Faxén force correction will be based on the volume of the particle. Using the expression for the added mass force as reported in literature $[15,28,32]$ and using volume averaging results in the added mass force for a solid particle

$$
\boldsymbol{F}_{\mathrm{AM}}=\iiint_{V_{\mathrm{p}}} \widetilde{C}_{\mathrm{AM}} \widetilde{\rho}_{\mathrm{c}}\left\{\frac{\widetilde{\mathrm{D} \boldsymbol{u}}}{\mathrm{D} t}-\frac{\mathrm{d}}{\mathrm{d} t}\left(\boldsymbol{v}+\boldsymbol{\omega}_{p} \times \boldsymbol{\chi}\right)\right\} \mathrm{d} V,
$$


The added mass coefficient for a spherical particle in inviscid flow equals $C_{\mathrm{AM}}=$ $\frac{1}{2}$, [4]. This added mass coefficient has been shown to be accurate for a wide range of Reynolds numbers [28]. Similar to the volume force and the stress divergence force, the added mass force, is

$$
\begin{aligned}
\frac{\boldsymbol{F}_{\mathrm{AM}}}{m} & =\frac{C_{\mathrm{AM}}}{\widehat{\rho}}\left\{\frac{\mathrm{D} \boldsymbol{u}}{\mathrm{D} t}-\frac{\mathrm{d} \boldsymbol{v}}{\mathrm{d} t}\right\}+\frac{D^{2}}{40} \nabla^{2}\left[\frac{C_{\mathrm{AM}}}{\widehat{\rho}} \frac{\mathrm{D} \boldsymbol{u}}{\mathrm{D} t}\right] \\
& -\frac{D^{2}}{40}\left\{\frac{\mathrm{d} \boldsymbol{v}}{\mathrm{d} t} \nabla^{2} \frac{C_{\mathrm{AM}}}{\widehat{\rho}}+2 \frac{\mathrm{d} \boldsymbol{\omega}_{\mathrm{p}}}{\mathrm{d} t} \times \nabla \frac{C_{\mathrm{AM}}}{\widehat{\rho}}\right\}+\mathcal{O}\left(\frac{\mathcal{F}}{m}\left\{\frac{D}{\mathcal{L}}\right\}^{4}\right) .
\end{aligned}
$$

Moreover, a volume averaged force approach introduces a torque on the particle as well, as the centre of the added mass force does not necessarily coincide with the centre of mass of the particle. The torque due to the added mass force then becomes

$$
\begin{array}{r}
\frac{\boldsymbol{T}_{\mathrm{AM}}}{I_{\mathrm{p}}}=\left\{\frac{\mathrm{d} \boldsymbol{v}}{\mathrm{d} t}-\frac{\mathrm{D} \boldsymbol{u}}{\mathrm{D} t}\right\} \times \nabla \frac{C_{\mathrm{AM}}}{2 \widehat{\rho}}+\frac{C_{\mathrm{AM}}}{2 \widehat{\rho}}\left\{\boldsymbol{\nabla} \times \frac{\mathrm{D} \boldsymbol{u}}{\mathrm{D} t}-2 \frac{\mathrm{d} \boldsymbol{\omega}_{\mathrm{p}}}{\mathrm{d} t}\right\} \\
+\frac{5 D^{2}}{168}\left\{\frac{\mathrm{d} \boldsymbol{v}}{\mathrm{d} t} \times \nabla^{2} \nabla \frac{C_{\mathrm{AM}}}{2 \widehat{\rho}}-2 \frac{\mathrm{d} \boldsymbol{\omega}_{\mathrm{p}}}{\mathrm{d} t} \cdot\left[\nabla^{2} \frac{C_{\mathrm{AM}}}{2 \widehat{\rho}} \mathbf{U}-\nabla \nabla \frac{C_{\mathrm{AM}}}{2 \widehat{\rho}}\right]\right\} \\
+\frac{5 D^{2}}{168} \nabla^{2} \boldsymbol{\nabla} \times\left[\frac{C_{\mathrm{AM}}}{2 \widehat{\rho}} \frac{\mathrm{D} \boldsymbol{u}}{\mathrm{D} t}\right]+\mathcal{O}\left(\frac{\mathcal{T}}{I_{\mathrm{p}}}\left\{\frac{D}{\mathcal{L}}\right\}^{4}\right) .
\end{array}
$$

\subsection{Lift forces}

The Saffman lift force and the Magnus lift force represent phenomena related to rotation of the particle and to rotation in the flow field. Figure 3.1 shows the two forces and the effects they represent.

The Saffman lift force captures the effect that vorticity in the flow field has on the force on a particle. Vorticity causes a velocity distribution that varies along the surface of the particle. The velocity distribution causes a varying pressure distribution at the surface of the particle resulting in a lift force. The direction of this force is perpendicular to the plane spanned by the vorticity vector and the relative velocity vector.

The Magnus lift force represents the effect of the flow field being deflected by the rotation of the particle relative to the vorticity. Relative rotation of the particle causes a change in angle between the flow ahead of the particle and the 

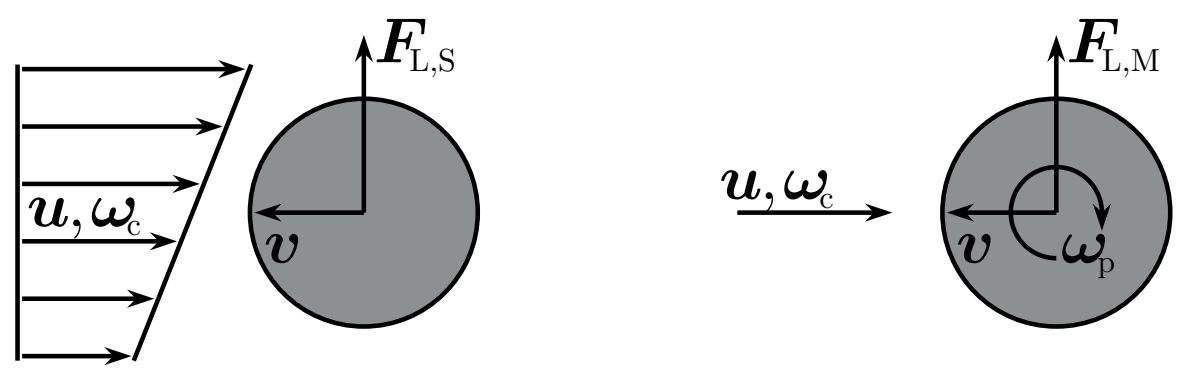

Figure 3.1: Vorticity in the flow field causes Saffman lift (left), while relative particle rotation causes Magnus lift (right).

flow in the wake of the particle. This deflection is caused by friction between the surface of the particle and the fluid. Some authors use the relative rotation for the Magnus lift [15], while others use the absolute rotation of the particle [28]. For the cases studied in this research, both methods have been investigated, and differences between the two approaches appear to be negligible. An approach using relative rotation will be used for results obtained taking into account the Magnus lift term.

\subsubsection{Saffman lift}

The Saffman lift force is an integration over the surface of a particle of the part of the pressure distribution caused by vorticity in the flow field. Using the divergence theorem the surface integral is converted to a volume integration, introducing the crossproduct of the curl of the flow field and the relative velocity of the local flow with respect to the average velocity of the particle. The expression of the lift force, following literature [29, 15, 28], is

$$
\boldsymbol{F}_{\mathrm{L}, \mathrm{S}}=\frac{9.66}{\pi} \iiint_{V_{\mathrm{p}}} \frac{C_{\mathrm{L}, \mathrm{S}}}{C_{\mathrm{L}, \mathrm{S}, \mathrm{Saff}}}(\widetilde{\alpha}, \widetilde{\operatorname{Re}}) \frac{\sqrt{2} \widetilde{\rho}_{\mathrm{c}}}{\sqrt{\widetilde{\alpha} \widetilde{\operatorname{Re}}}} \widetilde{\boldsymbol{\omega}}_{\mathrm{c}} \times\{\boldsymbol{v}-\widetilde{\boldsymbol{u}}\} \mathrm{d} V,
$$

with the vorticity of the flow field $\boldsymbol{\omega}_{\mathrm{c}}$ as defined in equation (3.9), and the local Reynolds and shear number, similarly to the particle Reynolds and shear number in equations (3.7) and (3.15), respectively defined as

$$
\widetilde{\operatorname{Re}} \equiv \frac{D\left\|\boldsymbol{v}+\boldsymbol{\omega}_{\mathrm{p}} \times \boldsymbol{\chi}-\widetilde{\boldsymbol{u}}\right\|}{\widetilde{\nu}}
$$




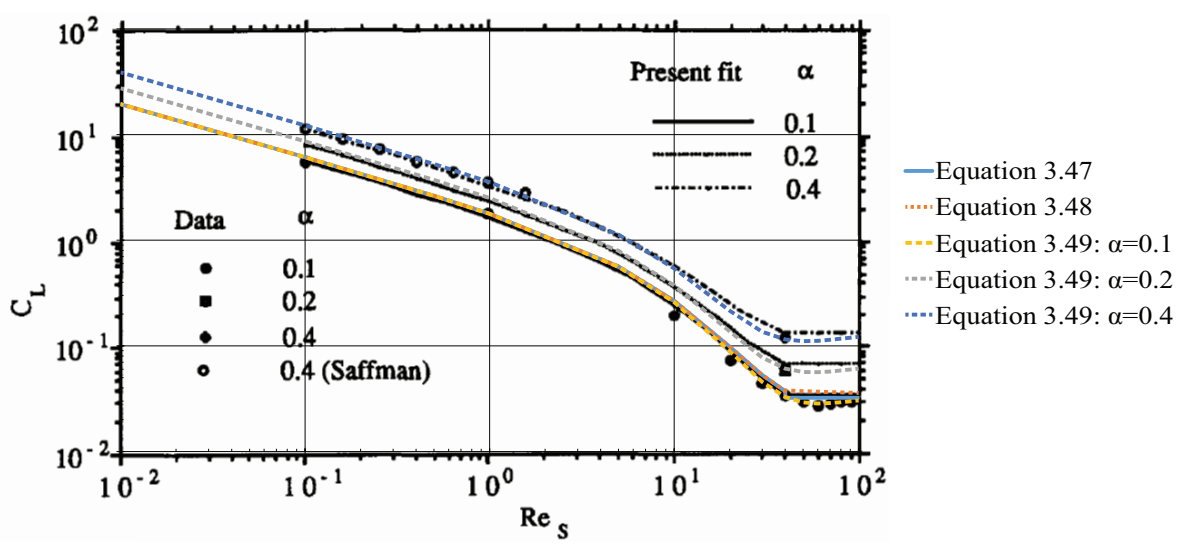

Figure 3.2: The different expressions for the Saffman lift coefficient presented superposed on the figure of the lift coefficient presented in Mei (1992) [29]. Note that $\operatorname{Re}_{\mathrm{S}}$ is equal to the diameter based particle Reynolds number Re, and $C_{\mathrm{L}}$ is the Saffman lift coefficient $C_{\mathrm{L}, \mathrm{S}}$.

$$
\widetilde{\alpha} \equiv \frac{D\left\|\boldsymbol{\omega}_{\mathrm{c}}\right\|}{2\left\|\boldsymbol{v}+\boldsymbol{\omega}_{\mathrm{p}} \times \boldsymbol{\chi}-\widetilde{\boldsymbol{u}}\right\|}
$$

The ratio of the Saffman lift coefficient, valid for a shear number, defined in equation (3.15), of $0.005<\alpha<0.4$, as well as for a larger range of Reynolds numbers, and the original Saffman lift coefficient for the viscous flow limit is $[29,15]$

$$
\frac{C_{\mathrm{L}, \mathrm{S}}}{C_{\mathrm{L}, \mathrm{S}, \mathrm{Saff}}}(\alpha, \mathrm{Re})= \begin{cases}0.3314 \sqrt{\alpha}\left(1-\mathrm{e}^{-\frac{\mathrm{Re}}{10}}\right)+\mathrm{e}^{-\frac{\mathrm{Re}}{10}} & \mathrm{Re} \leq 40 \\ 0.0524 \sqrt{\alpha \mathrm{Re}} & \mathrm{Re}>40\end{cases}
$$

Note that $0.0524 \approx \frac{\pi}{60}$ and is identical when evaluating $\frac{\pi}{60}$ up to the same number of significant numbers. Moreover, $0.3314 \approx 0.0524 \sqrt{40}$ up to the same number of significant numbers, and approximately equal to $\frac{\pi}{60} \sqrt{40}$. Furthermore, in equation (3.47) a jump is present at $R e=40$ that does not show up in results presented by Mei (1992) [29]. Therefore, the expression used might well have been

$$
\frac{C_{\mathrm{L}, \mathrm{S}}}{C_{\mathrm{L}, \mathrm{S}, \mathrm{Saff}}}(\alpha, \mathrm{Re})= \begin{cases}\frac{\pi}{60} \sqrt{40 \alpha}\left(1-\mathrm{e}^{-\frac{\mathrm{Re}}{10}}\right)+\mathrm{e}^{-\frac{\mathrm{Re}}{10}} & \mathrm{Re} \leq 40 \\ \frac{\pi}{60} \sqrt{\alpha \operatorname{Re}}\left(1-\mathrm{e}^{-4}\right)+\mathrm{e}^{-4} & \mathrm{Re}>40\end{cases}
$$


Furthermore, we introduce a qualitative fit which captures the rising trend for high Reynolds numbers in a single expression

$$
\frac{C_{\mathrm{L}, \mathrm{S}}}{C_{\mathrm{L}, \mathrm{S}, \mathrm{Saff}}}(\alpha, \mathrm{Re})=\frac{\pi}{159} \sqrt{\alpha} \mathrm{Re}^{0.7}\left(1-\mathrm{e}^{-\frac{\mathrm{Re}}{11}}\right)+\mathrm{e}^{-\frac{\mathrm{Re}}{11}} .
$$

The resulting expressions for the Saffman lift coefficient are presented in figure 3.2 on top of the results presented by Mei (1992) [29]. It can be observed that equation (3.48) is continuous, while equation (3.47) has a jump at $\operatorname{Re}=40$. However, the derivative of equation (3.48) is still discontinuous at $\operatorname{Re}=40$. The qualitative fit in equation (3.49) is continuous in the entire region for which Re $>0$ in both its function and derivative. Moreover, it manages to capture the dip for $\alpha=0.1$ at $\operatorname{Re}=60$ as well as the rising trend for $\operatorname{Re}>60$.

A Taylor series expansion of equation (3.45) results in

$$
\begin{aligned}
\frac{\boldsymbol{F}_{\mathrm{L}, \mathrm{S}}}{m}=\frac{f_{\mathrm{L}, \mathrm{S}}}{\widehat{\rho}} \boldsymbol{\omega}_{\mathrm{c}} & \times \boldsymbol{w}+\frac{D^{2}}{40} \nabla^{2}\left[\frac{f_{\mathrm{L}, \mathrm{S}}}{\widehat{\rho}} \boldsymbol{u} \times \boldsymbol{\omega}_{\mathrm{c}}\right] \\
& -\frac{D^{2}}{40} \boldsymbol{v} \times \nabla^{2}\left[\frac{f_{\mathrm{L}, \mathrm{S}}}{\widehat{\rho}} \boldsymbol{\omega}_{\mathrm{c}}\right]+\mathcal{O}\left(\frac{\mathcal{F}}{m}\left\{\frac{D}{\mathcal{L}}\right\}^{4}\right),
\end{aligned}
$$

where the Saffman lift factor $f_{\mathrm{L}, \mathrm{S}}$ is defined as

$$
f_{\mathrm{L}, \mathrm{S}} \equiv f_{\mathrm{L}, \mathrm{S}}(\alpha, \operatorname{Re})=\frac{\sqrt{2}}{\pi} \frac{9.66}{\sqrt{\alpha \mathrm{Re}}} \frac{C_{\mathrm{L}, \mathrm{S}}}{C_{\mathrm{L}, \mathrm{S}, \mathrm{Saff}}}(\alpha, \mathrm{Re}),
$$

Similarly for the torque

$$
\begin{array}{r}
\frac{\boldsymbol{T}_{\mathrm{L}, \mathrm{S}}}{I_{\mathrm{p}}}=\boldsymbol{\nabla} \times\left[\frac{f_{\mathrm{L}, \mathrm{S}}}{2 \widehat{\rho}} \boldsymbol{\omega}_{\mathrm{c}} \times \boldsymbol{w}\right]+\frac{5 D^{2}}{168} \nabla^{2} \boldsymbol{\nabla} \times\left[\frac{f_{\mathrm{L}, \mathrm{S}}}{2 \widehat{\rho}} \boldsymbol{\omega}_{\mathrm{c}} \times \boldsymbol{w}\right] \\
+\boldsymbol{\mathcal { O }}\left(\frac{\mathcal{T}}{I_{\mathrm{p}}}\left\{\frac{D}{\mathcal{L}}\right\}^{4}\right)
\end{array}
$$

\subsubsection{Magnus lift}

Contrary to the Saffman lift force, the Magnus lift force depends on the local relative velocity. The Magnus lift force was derived by Rubinow and Keller (1951) [34]. Following literature [28, 17, 18] the Magnus lift force is expressed 
as

$$
\boldsymbol{F}_{\mathrm{L}, \mathrm{M}}=\frac{3}{4} \iiint_{V} C_{\mathrm{L}, \mathrm{M}}(\widetilde{\Omega}, \widetilde{\mathrm{Re}}) \widetilde{\rho}_{\mathrm{c}} \widetilde{\boldsymbol{\omega}}_{\mathrm{r}} \times\left\{\boldsymbol{v}+\boldsymbol{\omega}_{\mathrm{p}} \times \boldsymbol{\chi}-\widetilde{\boldsymbol{u}}\right\} \mathrm{d} V,
$$

with the Magnus lift coefficient

$$
\begin{aligned}
& C_{\mathrm{L}, \mathrm{M}}(\Omega, \mathrm{Re}) \\
& \quad=1-\left\{\frac{5}{8}+0.15[1-\tanh (0.28[\Omega-2])]\right\} \tanh (0.18 \sqrt{\mathrm{Re}}),
\end{aligned}
$$

where $\Omega$ is the spin number as defined in equation (3.14). The local spin number is

$$
\widetilde{\Omega} \equiv \frac{D\left\|\boldsymbol{\omega}_{\mathrm{p}}-\frac{1}{2} \widetilde{\boldsymbol{\omega}}_{\mathrm{c}}\right\|}{\left\|\boldsymbol{v}+\boldsymbol{\omega}_{\mathrm{p}} \times \boldsymbol{\chi}-\widetilde{\boldsymbol{u}}\right\|}
$$

Defining the Magnus lift factor as

$$
f_{\mathrm{L}, \mathrm{M}} \equiv \frac{3}{4} C_{\mathrm{L}, \mathrm{M}}(\Omega, \mathrm{Re})
$$

and employing a Taylor series expansion, equation (3.53) becomes

$$
\begin{aligned}
\frac{\boldsymbol{F}_{\mathrm{L}, \mathrm{M}}}{m}=\frac{f_{\mathrm{L}, \mathrm{M}}}{\widehat{\rho}} \boldsymbol{\omega}_{\mathrm{r}} \times \boldsymbol{w} & +\frac{D^{2}}{40}\left\{\nabla^{2}\left[\frac{f_{\mathrm{L}, \mathrm{M}}}{\hat{\rho}} \boldsymbol{\omega}_{\mathrm{r}} \times \boldsymbol{w}\right]-\frac{f_{\mathrm{L}, \mathrm{M}}}{\widehat{\rho}} \nabla \boldsymbol{\omega}_{\mathrm{c}} \cdot \boldsymbol{\omega}_{\mathrm{p}}\right\} \\
+ & \frac{2 D^{2}}{40} \boldsymbol{\omega}_{\mathrm{r}} \times\left[\boldsymbol{\omega}_{\mathrm{p}} \times \nabla \frac{f_{\mathrm{L}, \mathrm{M}}}{\widehat{\rho}}\right]+\mathcal{O}\left(\frac{\mathcal{F}}{m}\left\{\frac{D}{\mathcal{L}}\right\}^{4}\right),
\end{aligned}
$$

Similarly for the torque

$$
\begin{array}{r}
\frac{\boldsymbol{T}_{\mathrm{L}, \mathrm{S}}}{I_{\mathrm{p}}}=\left\{\frac{f_{\mathrm{L}, \mathrm{M}}}{2 \widehat{\rho}}+\frac{5 D^{2}}{168} \nabla^{2} \frac{f_{\mathrm{L}, \mathrm{M}}}{2 \widehat{\rho}}\right\} \boldsymbol{\omega}_{\mathrm{p}} \times \frac{\boldsymbol{\omega}_{\mathrm{c}}}{2}+\nabla \times\left[\frac{f_{\mathrm{L}, \mathrm{M}}}{2 \widehat{\rho}} \boldsymbol{\omega}_{\mathrm{r}} \times \boldsymbol{w}\right] \\
+\frac{5 D^{2}}{168} \boldsymbol{\omega}_{\mathrm{p}} \times\left\{\left(\nabla \frac{f_{\mathrm{L}, \mathrm{M}}}{2 \widehat{\rho}}\right) \cdot \overline{\bar{\nabla} \boldsymbol{\omega}_{\mathrm{c}}}+\frac{f_{\mathrm{L}, \mathrm{M}}}{2 \widehat{\rho}} \nabla^{2} \frac{\boldsymbol{\omega}_{\mathrm{c}}}{2}-\boldsymbol{\omega}_{\mathrm{r}} \cdot \nabla \nabla \frac{f_{\mathrm{L}, \mathrm{M}}}{2 \widehat{\rho}}\right\} \\
\frac{5 D^{2}}{168} \nabla^{2} \boldsymbol{\nabla} \times\left[\frac{f_{\mathrm{L}, \mathrm{M}}}{\widehat{\rho}} \boldsymbol{\omega}_{\mathrm{r}} \times \boldsymbol{w}\right]+\mathcal{O}\left(\frac{\mathcal{T}}{I_{\mathrm{p}}}\left\{\frac{D}{\mathcal{L}}\right\}^{4}\right),
\end{array}
$$

where

$$
\overline{\overline{\nabla \omega_{\mathrm{c}}}} \equiv \frac{1}{2}\left\{\boldsymbol{\nabla} \boldsymbol{\omega}_{\mathrm{c}}+\left(\boldsymbol{\nabla} \boldsymbol{\omega}_{\mathrm{c}}\right)^{\mathrm{T}}\right\},
$$

i.e. the symmetric vorticity gradient tensor. 


\subsection{Drag force}

The drag force approximates the effect that the particle tends to follow the motion of the fluid. Both viscous drag due to boundary layer development as well as pressure drag due to flow separation at the surface of the particle is included. Expressions for the drag force commonly involve a drag coefficient that represents both viscous and pressure effects. The drag force is due to a difference between the velocity of the surface of the particle and the velcoity of the macroscopic flow field. In terms of the drag coefficient, it is expressed as

$$
\boldsymbol{F}_{\mathrm{D}}=-\frac{1}{8} \iint_{\partial V} C_{\mathrm{D}}(\widetilde{\operatorname{Re}}) \widetilde{\rho}_{\mathrm{c}}\left\|\boldsymbol{v}+\boldsymbol{\omega}_{\mathrm{p}} \times \boldsymbol{\chi}-\widetilde{\boldsymbol{u}}\right\|\left(\boldsymbol{v}+\boldsymbol{\omega}_{\mathrm{p}} \times \boldsymbol{\chi}-\widetilde{\boldsymbol{u}}\right) \mathrm{d} S,
$$

with the local Reynolds number $\widetilde{R e}$ as defined in equation (3.46a). The drag coefficient corresponding to a sphere in a stationary uniform flow in the limit of the Reynolds number to zero, so-called Stokes flow, is

$$
C_{\mathrm{D}, \text { Stokes }}(\mathrm{Re})=\frac{24}{\operatorname{Re}} \text {. }
$$

\subsubsection{Drag coefficient and drag factor}

The Reynolds dependent drag coefficient will now be written in terms of the drag factor. The drag factor is the ratio of the drag coefficient and the Stokes drag coefficient

$$
f_{\mathrm{D}}=f_{\mathrm{D}}(\mathrm{Re}) \equiv \frac{\mathrm{Re}}{24} C_{\mathrm{D}}
$$

Therefore, the force in terms of the drag factor is

$$
\boldsymbol{F}_{\mathrm{D}}=-3 D \iint_{\partial V} f(\widetilde{\operatorname{Re}}) \frac{\widetilde{\rho}_{c}}{\widetilde{\tau}_{\mathrm{d}}}\left(\boldsymbol{v}+\boldsymbol{\omega}_{\mathrm{p}} \times \boldsymbol{\chi}-\widetilde{\boldsymbol{u}}\right) \mathrm{d} S
$$

In the literature, many correlations for the drag for a sphere in a stationary uniform flow exist. Some are analytical or semi-analytical, while others are empirical. In commercial CFD codes (see e.g. [3]), the commonly used drag coefficient is an extension of the expression proposed by Schiller \& Naumann in $1933[35] f_{\mathrm{D}, \mathrm{SN}}$, extended to the Newton regime in which $C_{\mathrm{D}, \text { Newton }}(\mathrm{Re})=0.44$. The drag factor is described by

$$
f_{\mathrm{D}, \mathrm{CFD}}=\max \left(f_{\mathrm{D}, \mathrm{SN}}, 0.44 \frac{\mathrm{Re}}{24}\right)
$$




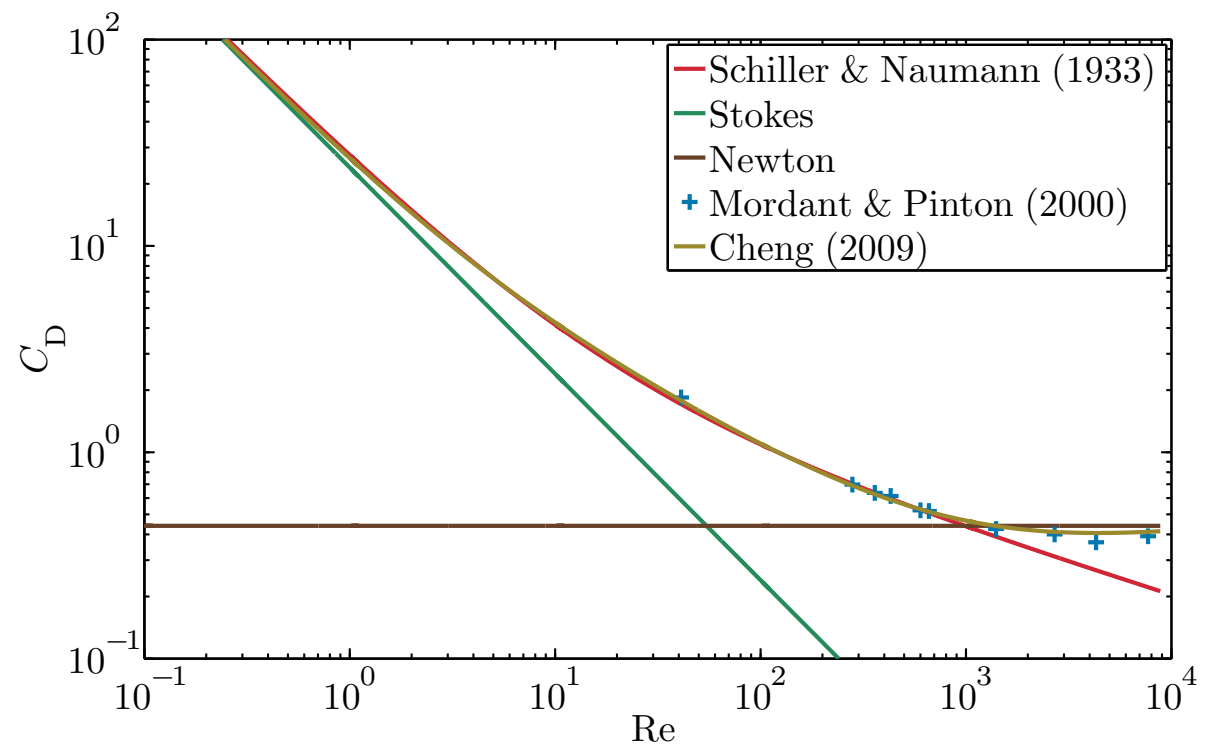

Figure 3.3: Drag coefficient as a function of Reynolds number for several drag correlations. Experimental data published by Mordant \& Pinton in 2000 [32] is used in this research

where

$$
f_{\mathrm{D}, \mathrm{SN}}=1+0.15 \mathrm{Re}^{0.687}
$$

In this research, the drag factor proposed by Cheng in 2009 [10] is used,

$$
f_{\mathrm{D}, \mathrm{Ch}}=(1+0.27 \mathrm{Re})^{0.43}+0.47 \frac{\mathrm{Re}}{24}\left[1-e^{-\left(0.04 \mathrm{Re}^{0.38}\right)}\right]
$$

Cheng obtained this drag correlation by a fit of a collection of drag data. This data had been the basis for many existing correlations. However, in 2003 Brown \& Lawler corrected this data for wall effects in order to obtain a more accurate error estimation [6]. Cheng's correlation, obtained using this corrected data, is shown to have higher accuracy than other similar expressions with respect to the data corrected for wall effects $[6,10]$.

The resulting drag curves of the correlations are presented in figure 3.3. The drag correlation by Cheng [10] smoothly transitions from the drag in the Stokes regime to the drag in the Newton regime. The drag correlation by Schiller \& 
Naumann [35] results in values for the drag coefficient for Reynolds numbers up to the Newton regime very similar to Cheng's expression. However, it does not capture the change to the Newton regime. Moreover, even with the adaptation as used in commercial CFD codes the drag coefficient clearly deviates from the drag coefficient by Cheng. Furthermore, the experimental data published by Mordant \& Pinton in 2000 [32] and used in this research, is closer to the drag coefficient proposed by Cheng, as shown in figure 3.3 Also, the drag factor proposed by Cheng is more convenient in numerical schemes. It has non-singular derivatives for all Reynolds numbers, while the first derivative of the drag factor proposed by Schiller \& Naumann is singular for $\mathrm{Re}=0$ and transition to the Newton regime introduces a jump in the first derivative of the expression.

\subsubsection{Approximation of the drag force}

With a Taylor series expansion, equation (3.60) becomes

$$
\begin{aligned}
\frac{\boldsymbol{F}_{\mathrm{D}}}{m}= & -18\left\{\frac{f_{\mathrm{D}}(\mathrm{Re})}{\widehat{\rho} \tau_{\mathrm{d}}} \boldsymbol{w}-\frac{D^{2}}{24} \frac{f_{\mathrm{D}}(\mathrm{Re})}{\widehat{\rho} \tau_{\mathrm{d}}} \nabla^{2} \boldsymbol{u}\right\} \\
& +\frac{3}{2} D^{2}\left\{\left(\boldsymbol{\nabla} \frac{f_{\mathrm{D}}(\mathrm{Re})}{\widehat{\rho} \tau_{\mathrm{d}}}\right) \cdot \overline{\overline{\nabla \boldsymbol{u}}}-\boldsymbol{\omega}_{\mathrm{r}} \times \nabla \frac{f_{\mathrm{D}}(\mathrm{Re})}{\widehat{\rho} \tau_{\mathrm{d}}}\right\} \\
& -\frac{3}{4} D^{2} \boldsymbol{w} \nabla^{2} \frac{f_{\mathrm{D}}(\mathrm{Re})}{\widehat{\rho} \tau_{\mathrm{d}}}+\boldsymbol{O}\left(\frac{\mathcal{F}}{m}\left\{\frac{D}{\mathcal{L}}\right\}^{4}\right) .
\end{aligned}
$$

The gradient and the Laplacian of the drag factor at the particle location in equation (3.67) are

$$
\begin{gathered}
\left.\boldsymbol{\nabla} f_{\mathrm{D}}(\mathrm{Re})\right|_{\boldsymbol{\chi}=\mathbf{0}}=f_{\mathrm{D}}^{\prime}(\mathrm{Re}) \boldsymbol{\nabla R e}, \\
\left.\nabla^{2} f_{\mathrm{D}}(\mathrm{Re})\right|_{\boldsymbol{\chi}=\mathbf{0}}=f_{\mathrm{D}}^{\prime}(\mathrm{Re}) \nabla^{2} \operatorname{Re}+f_{\mathrm{D}}^{\prime \prime}(\mathrm{Re})\|\boldsymbol{\nabla R e}\|^{2},
\end{gathered}
$$

respectively, with

$$
\begin{aligned}
&\left.\nabla \operatorname{Re}\right|_{\boldsymbol{\chi}=\mathbf{0}}=\operatorname{Re}\left\{\frac{\boldsymbol{w}}{\|\boldsymbol{w}\|} \times \frac{2 \boldsymbol{\omega}_{\mathrm{r}}-\boldsymbol{\omega}_{\mathrm{c}}}{2\|\boldsymbol{w}\|}-\frac{\boldsymbol{w}}{\|\boldsymbol{w}\|} \cdot \frac{\boldsymbol{\nabla} \boldsymbol{u}}{\|\boldsymbol{w}\|}-\frac{\boldsymbol{\nabla} \nu}{\nu}\right\}, \\
&\left.\nabla^{2} \operatorname{Re}\right|_{\boldsymbol{\chi}=\mathbf{0}}=\operatorname{Re} \frac{(\boldsymbol{\nabla} \boldsymbol{u})^{\mathrm{T}}: \boldsymbol{\nabla} \boldsymbol{u}-\boldsymbol{w} \cdot \boldsymbol{\nabla} \boldsymbol{u}-2 \boldsymbol{\omega}_{\mathrm{r}} \cdot \boldsymbol{\omega}_{\mathrm{c}}}{\|\boldsymbol{w}\|} \\
&-\operatorname{Re}\left\{\left\|\frac{\boldsymbol{\nabla R e}}{\operatorname{Re}}\right\|^{2}+4 \frac{\boldsymbol{\nabla R e}}{\operatorname{Re}} \cdot \frac{\boldsymbol{\nabla} \nu}{\nu}+\left\|\frac{\boldsymbol{\nabla} \nu}{\nu}\right\|^{2}+\frac{\nabla^{2} \nu}{\nu}\right\} .
\end{aligned}
$$




\subsubsection{Approximation of the torque due to drag}

Similarly for the torque of the drag

$$
\begin{aligned}
& \frac{\boldsymbol{T}_{\mathrm{D}}}{I_{\mathrm{p}}}=-15\left\{\frac{f_{\mathrm{D}}}{\widehat{\rho} \tau_{\mathrm{d}}}\left[2 \boldsymbol{\omega}_{\mathrm{r}}-\frac{D^{2}}{24} \nabla^{2} \boldsymbol{\omega}_{\mathrm{c}}\right]+\boldsymbol{w} \times \boldsymbol{\nabla} \frac{f_{\mathrm{D}}}{\hat{\rho} \tau_{\mathrm{d}}}\right\} \\
& -15 \frac{D^{2}}{24}\left\{2 \boldsymbol{\omega}_{\mathrm{r}} \nabla^{2} \frac{f_{\mathrm{D}}}{\widehat{\rho} \tau_{\mathrm{d}}}+\left(4 \boldsymbol{\omega}_{\mathrm{r}}+\boldsymbol{\omega}_{\mathrm{c}}\right) \cdot\left[\nabla^{2} \frac{f_{\mathrm{D}}}{\widehat{\rho} \tau_{\mathrm{d}}} \mathbf{U}-\nabla \nabla \frac{f_{\mathrm{D}}}{\widehat{\rho} \tau_{\mathrm{d}}}\right]\right\} \\
& +15 \frac{D^{2}}{24}\left\{2 \boldsymbol{\nabla} \frac{f_{\mathrm{D}}}{\widehat{\rho} \tau_{\mathrm{d}}} \cdot \boldsymbol{\nabla} \boldsymbol{\omega}_{\mathrm{c}}+\nabla \frac{f_{\mathrm{D}}}{\widehat{\rho} \tau_{\mathrm{d}}} \times \nabla^{2} \boldsymbol{u}\right\} \\
& +15 \frac{D^{2}}{24} \sum_{i=1}^{3}\left\{\left(\boldsymbol{\nabla} \frac{\partial}{\partial x_{i}} \frac{f_{\mathrm{D}}}{\widehat{\rho} \tau_{\mathrm{d}}}\right) \times\left(\nabla u_{i}+\frac{\partial \boldsymbol{u}}{\partial x_{i}}\right)\right\} \\
& +15 \frac{D^{2}}{24} \boldsymbol{w} \times \nabla^{2} \boldsymbol{\nabla} \frac{f_{\mathrm{D}}}{\widehat{\rho} \tau_{\mathrm{d}}}+\boldsymbol{\mathcal { O }}\left(\frac{\mathcal{T}}{I_{\mathrm{p}}}\left\{\frac{D}{\mathcal{L}}\right\}^{4}\right) .
\end{aligned}
$$

However, in literature $[15,18]$ the torque due to relative rotation is expressed as

$$
\frac{\boldsymbol{T}_{\boldsymbol{\omega}_{\mathrm{r}}}}{I_{\mathrm{p}}}=-\frac{120.6}{\pi \hat{\rho} \tau_{\mathrm{d}}}\left(1+0.1005 \sqrt{\tau_{\mathrm{d}}\left\|\boldsymbol{\omega}_{\mathrm{r}}\right\|}\right) \boldsymbol{\omega}_{\mathrm{r}},
$$

which is similar to the first term in the torque due to higher order terms in the drag. If higher order terms are taken into account for the forces, it should be investigated if the torque due to the drag replaces the torque due to relative rotation.

\subsection{History force}

The expression of the drag and lift forces on a particle are based on steady state motion, in which relative velocity, vorticity and rate of rotation as well as the wake of the particle do not vary in time. In other words, they depend only on the instantaneous values for Reynolds number, velocity, vorticity and so on. However, for many applications, such as separation processes, residence times will be short and effects due to acceleration of particles might be important. Moreover, complex flow fields will result in oscillations in relative velocity as well as vorticity and rate of rotation. This implies that the boundary layer as well as the wake will not be steady. The history force is the force that accounts for the lagging in time of a transient boundary layer, as well as that of the 
particle wake. To capture this effect, integration over the entire time-history of the motion of the particle is required. This integral not only requires an increasing amount of computational effort for increasing simulation times, also discretizing the integral requires careful analysis.

In this research, the history force will only take into account the effects of the time-dependent drag force. However, a similar approach can be used to account for transient effects of the lift forces. The history force is

$$
\frac{\boldsymbol{F}_{\mathrm{H}}}{m}=-18 \int_{-\infty}^{t} \iint_{\partial V} \frac{\widetilde{K}(\tau, t)}{\widetilde{\rho} \widetilde{\tau}_{\mathrm{d}}} \frac{\mathrm{d} \widetilde{\boldsymbol{w}}}{\mathrm{d} \tau} \mathrm{d} S \mathrm{~d} \tau,
$$

where the kernel $K(\tau, t)$ in the integral for the history force relates the influence of the relative acceleration of the particle at time $\tau$ on the history force at time $t$. Generally, the influence of the relative acceleration decreases over time. Therefore, the kernel will generally decrease for increasing history time $(t-\tau)$.

\subsubsection{Basset kernel for the history force}

The history force is often called the Basset force, or the Basset history force. The Basset force is the history force with a specific kernel. The Basset history kernel is related to Stokes' second problem of a plate oscillating in its plane. For a sphere, under the assumption of Stokes flow, the Basset history kernel is obtained by integration of the error function [15]. The resulting kernel is

$$
K_{\mathrm{B}}(\tau, t) \equiv \frac{1}{\sqrt{4 \pi}} \frac{1}{\sqrt{T_{\mathrm{B}}(\tau, t)}},
$$

in which the dimensionless Basset history time is defined as

$$
T_{\mathrm{B}}(\tau, t) \equiv \frac{t-\tau}{\tau_{\mathrm{d}}} .
$$

It can be observed that the kernel decays with the square root of the history time. Moreover, the Basset kernel has a square root singularity at $\tau=t$, which is the upper bound of the time-integral. When treating the integral numerically, this singularity requires analysis.

\subsubsection{Influence of inertia on the history kernel}

It has been observed that, different from the behaviour of the Basset kernel, the long time behaviour of the kernel should show a faster decay than the square root 
$[31,28,32]$, i.e. closer to quadratic decay. As a consequence, using the Basset kernel for non-Stokesian flow leads to significant over-prediction of the history force. A kernel was proposed by Mei and Mei \& Adrian in 1991 and 1992 [31, 30] for the long time behaviour, which takes into account effects due to inertia. This kernel was subsequently refined by Lawrence \& Mei on a semi-analytical basis in 1995 [27] connecting the Reynolds number dependent behaviour to the drag factor, resulting in

$$
K_{\mathrm{L} \& \mathrm{M}, T \gg 1}(\tau, t) \equiv \frac{3\left[f_{\mathrm{D}}(t)+\operatorname{Re}(t) f_{\mathrm{D}}^{\prime}(t)\right] f_{\mathrm{D}}^{\prime}(\tau)}{\left[\operatorname{Re}(t) T_{\mathrm{H}}(\tau, t)\right]^{2}},
$$

with the Reynolds number dependent history time defined as

$$
T_{\mathrm{H}}(\tau, t) \equiv \frac{1}{\tau_{\mathrm{d}}} \int_{\tau}^{t} \frac{\operatorname{Re}(s)}{\operatorname{Re}(t)} \mathrm{d} s
$$

and where

$$
\left.f_{\mathrm{d}}^{\prime} \equiv \frac{\mathrm{d} f_{\mathrm{D}}}{\mathrm{d} \operatorname{Re}}\right|_{\operatorname{Re}(t)} .
$$

Note that the Reynolds dependent history time reduces to the Basset history time for the limit of the Reynolds number to zero, i.e. for Stokes Flow.

In literature several kernels have been developed that combine the short term square-root decay and the long term quadratic decay based on the kernels proposed by Mei, Mei \& Adrian and Lawrence \& Mei. Short time and long time behaviour can be combined by separating the integration with respect to time employing a characteristic history time $\tau_{\mathrm{H}}$, in a short time integral from $t-\tau_{\mathrm{H}}$ up to $t$ and a long time integral from $-\infty$ up to $t-\tau_{\mathrm{H}}$ [27]. The effect of the long time behaviour of the kernel has also been approximated by using the Basset kernel and only integrating over a certain reduced timewindow [16]. The resulting approximation of the history force posesses increased accuracy with respect to the Basset history force while it simultaneously reduces computational cost significantly. The approach used in the present research is to capture the short-time and long-time behaviour in a single kernel. Starting from kernels proposed in literature by Mei \& Adrian, Lawrence \& Mei and Loth \& Dorgan $[30,27,16,28]$ the kernel has been adapted $[17,18,19,20]$ comparing the resulting particle behaviour to experimental data from literature [32]. The 
kernel proposed in the present research is

$$
K_{\mathrm{H}}(\tau, t) \equiv \frac{4}{\pi} \frac{f_{\mathrm{H}}(\tau, t)}{\left[\left[g(\tau, t) T_{\mathrm{H}}(\tau, t)\right]^{\frac{1}{4}}+\operatorname{Re}(t) T_{\mathrm{H}}(\tau, t)\right]^{2}},
$$

with the history factor $f_{\mathrm{H}}$ defined as

$$
f_{\mathrm{H}}(\tau, t) \equiv \frac{3}{4} \pi\left\{f_{\mathrm{D}}(t)+\operatorname{Re}(t) f_{\mathrm{D}}^{\prime}(t)\right\} f_{\mathrm{D}}^{\prime}(\tau)
$$

the history time as defined in equation (3.76), and the factor $g(\tau, t)$ defined such that the Basset kernel is obtained in the limit of Stokes flow, but also that prediction of the history force matches the experimental data. This research determines $g(\tau, t)$ to be

$$
g(\tau, t) \equiv 36 \pi\left[f_{\mathrm{D}}^{\prime}(\tau)\right]^{2}\left\{1+\left[\frac{h(\tau)[\operatorname{Re}(\tau)]^{2}}{\operatorname{Re}(t) T_{\mathrm{H}}(\tau, t)} \int_{\tau}^{t} \frac{\mathrm{d} \operatorname{Re} f_{\mathrm{D}}}{\mathrm{d} s} \mathrm{~d} s\right]^{2}\right\}
$$

where

$$
h(\tau) \equiv\left\{1-\mathrm{e}^{-[0.03 \operatorname{Re}(\tau)]^{2}}\right\}\left\{\operatorname{Re}(\tau)\left[\frac{11.5 f_{\mathrm{D}}^{\prime}(\tau)}{f_{\mathrm{D}}(\tau)}\right]^{6}+\frac{\left[f_{\mathrm{D}}^{\prime}(\tau)\right]^{6}}{4}\right\}
$$

The proposed kernel combines both short term and long term behaviour of the kernel in the history force. Moreover, the history time, proposed by Mei and Lawrence [27], implies that for low Reynolds numbers the effects of the history do not decline as fast as for high Reynolds number flow. Furthermore, the Basset kernel is obtained in the limit of the Reynolds number tending to zero. 


\subsubsection{Approximation of the history force and torque}

Similar to the drag force, the history force and the corresponding torque can be approximated by

$$
\begin{aligned}
& \frac{\boldsymbol{F}_{\mathrm{H}}}{m}=-18 \int_{-\infty}^{t} \frac{K(\tau, t)}{\widehat{\rho} \tau_{\mathrm{d}}}\left\{\frac{\mathrm{d} \boldsymbol{w}}{\mathrm{d} \tau}-\frac{D^{2}}{24} \frac{\mathrm{d} \nabla^{2} \boldsymbol{u}}{\mathrm{d} \tau}\right\} \mathrm{d} \tau \\
&+\frac{3}{2} D^{2} \int_{-\infty}^{t}\left(\nabla \frac{K(\tau, t)}{\widehat{\rho} \tau_{\mathrm{d}}}\right) \cdot \frac{\mathrm{d} \overline{\bar{\nabla} \boldsymbol{u}}}{\mathrm{d} \tau}-\frac{\mathrm{d} \boldsymbol{\omega}_{\mathrm{r}}}{\mathrm{d} \tau} \times \nabla \frac{K(\tau, t)}{\widehat{\rho} \tau_{\mathrm{d}}} \mathrm{d} \tau \\
&-\frac{3}{4} D^{2} \int_{-\infty}^{t} \frac{\mathrm{d} \boldsymbol{w}}{\mathrm{d} \tau} \nabla^{2} \frac{K(\tau, t)}{\widehat{\rho} \tau_{\mathrm{d}}} \mathrm{d} \tau+\mathcal{O}\left(\frac{\mathcal{F}}{m}\left\{\frac{D}{\mathcal{L}}\right\}^{4}\right),
\end{aligned}
$$

and

$$
\begin{array}{|c|}
\frac{\boldsymbol{T}_{\mathrm{H}}}{I_{\mathrm{p}}}=-15 \int_{-\infty}^{t} \frac{K}{\widehat{\rho} \tau_{\mathrm{d}}}\left\{2 \frac{\mathrm{d} \boldsymbol{\omega}_{\mathrm{r}}}{\mathrm{d} \tau}-\frac{D^{2}}{24} \frac{\mathrm{d} \nabla^{2} \boldsymbol{\omega}_{\mathrm{c}}}{\mathrm{d} \tau}\right\}+\frac{\mathrm{d} \boldsymbol{w}}{\mathrm{d} \tau} \times \nabla \frac{K}{\widehat{\rho} \tau_{\mathrm{d}}} \mathrm{d} \tau \\
-15 \frac{D^{2}}{24} \int_{-\infty}^{t} 2 \frac{\mathrm{d} \boldsymbol{\omega}_{\mathrm{r}}}{\mathrm{d} \tau} \nabla^{2} \frac{K}{\widehat{\rho} \tau_{\mathrm{d}}}+\frac{\mathrm{d}}{\mathrm{d} \tau}\left(4 \boldsymbol{\omega}_{\mathrm{r}}+\boldsymbol{\omega}_{\mathrm{c}}\right) \cdot\left[\nabla^{2} \frac{K}{\widehat{\rho} \tau_{\mathrm{d}}} \mathbf{U}-\nabla \nabla \frac{K}{\widehat{\rho} \tau_{\mathrm{d}}}\right] \mathrm{d} \tau \\
+15 \frac{D^{2}}{24} \int_{-\infty}^{t} 2 \boldsymbol{\nabla} \frac{K}{\widehat{\rho} \tau_{\mathrm{d}}} \cdot \frac{\mathrm{d} \boldsymbol{\nabla} \boldsymbol{\omega}_{\mathrm{c}}}{\mathrm{d} \tau}-\boldsymbol{\nabla} \frac{K}{\widehat{\rho} \tau_{\mathrm{d}}} \times \frac{\mathrm{d} \nabla^{2} \boldsymbol{u}}{\mathrm{d} \tau} \mathrm{d} \tau \\
+15 \frac{D^{2}}{24} \int_{-\infty}^{t} \sum_{i=1}^{3}\left\{\left(\nabla \frac{\partial}{\partial x_{i}} \frac{K}{\widehat{\rho} \tau_{\mathrm{d}}}\right) \times \frac{\mathrm{d}}{\mathrm{d} \tau}\left(\nabla u_{i}+\frac{\partial \boldsymbol{u}}{\partial x_{i}}\right)\right\} \mathrm{d} \tau \\
+15 \frac{D^{2}}{24} \int_{-\infty}^{t} \frac{\mathrm{d} \boldsymbol{w}}{\mathrm{d} \tau} \times \nabla^{2} \boldsymbol{\nabla} \frac{K}{\widehat{\rho} \tau_{\mathrm{d}}} \mathrm{d} \tau+\mathcal{O}\left(\frac{\mathcal{T}}{I_{\mathrm{p}}}\left\{\frac{D}{\mathcal{L}}\right\}^{4}\right)
\end{array}
$$

\subsubsection{Dealing with minus infinity}

The integral in equation (3.72) requires knowledge of the particle motion during its entire lifetime. However, this knowledge is generally not available. For 
a particle with constant shape and volume, without loss of generality, using partial integration for integral times for the time interval $-\infty \leq \tau<t_{0}$, the history force can be expressed as

$$
\begin{aligned}
& \frac{\boldsymbol{F}_{\mathrm{H}}}{m}=- 18 \int_{t_{0}}^{t} \frac{K}{\widehat{\rho} \tau_{\mathrm{d}}}\left[\frac{\mathrm{d} \boldsymbol{w}}{\mathrm{d} \tau}-\frac{D^{2}}{24} \frac{\mathrm{d} \nabla^{2} \boldsymbol{u}}{\mathrm{d} \tau}\right]+\frac{D^{2}}{24} \frac{\mathrm{d} \boldsymbol{w}}{\mathrm{d} \tau} \nabla^{2} \frac{K}{\widehat{\rho} \tau_{\mathrm{d}}} \mathrm{d} \tau \\
&-18\left\{\frac{K_{0}(t)}{\widehat{\rho}_{0} \tau_{\mathrm{d}, 0}}\left[\boldsymbol{w}_{0}-\frac{D^{2}}{24} \nabla^{2} \boldsymbol{u}_{0}\right]+\frac{D^{2}}{24} \boldsymbol{w}_{0} \nabla^{2} \frac{K_{0}(t)}{\widehat{\rho}_{0} \tau_{\mathrm{d}, 0}}\right\} \\
&+\frac{3}{2} D^{2} \int_{t_{0}}^{t}\left(\boldsymbol{\nabla} \frac{K}{\widehat{\rho} \tau_{\mathrm{d}}}\right) \cdot \frac{\mathrm{d} \overline{\overline{\boldsymbol{\nabla}} \boldsymbol{u}}}{\mathrm{d} \tau}-\frac{\mathrm{d} \boldsymbol{\omega}_{\mathrm{r}}}{\mathrm{d} \tau} \times \boldsymbol{\nabla} \frac{K}{\widehat{\rho} \tau_{\mathrm{d}}} \mathrm{d} \tau+\boldsymbol{f}_{\mathrm{H}, \tau<t_{0}} \\
&+\frac{3}{2} D^{2}\left\{\nabla \frac{K_{0}(t)}{\widehat{\rho}_{0} \tau_{\mathrm{d}, 0}} \cdot \overline{\bar{\nabla}}_{0}-\boldsymbol{\omega}_{\mathrm{r}, 0} \times \boldsymbol{\nabla} \frac{K_{0}(t)}{\widehat{\rho}_{0} \tau_{\mathrm{d}, 0}}\right\}+\mathcal{O}\left(\frac{\mathcal{F}}{m}\left\{\frac{D}{\mathcal{L}}\right\}^{4}\right),
\end{aligned}
$$

with $K_{0}(t) \equiv K\left(t_{0}, t\right)$, and with the subscript 0 indicating that fluid properties and flow velocities are taken at time $\tau=t_{0}$. The remaining influence of the contribution of the integral in the time interval $\infty \leq \tau<t_{0}$ is captured in $\boldsymbol{f}_{\mathrm{H}, \tau<t_{0}}$, which is defined as

$$
\begin{aligned}
& \boldsymbol{f}_{\mathrm{H}, \tau<t_{0}} \equiv 18 \int_{-\infty}^{t_{0}}\left[\boldsymbol{w}-\frac{D^{2}}{24} \nabla^{2} \boldsymbol{u}\right] \frac{\mathrm{d}}{\mathrm{d} \tau} \frac{K}{\widehat{\rho} \tau_{\mathrm{d}}}+\frac{D^{2}}{24} \boldsymbol{w} \frac{\mathrm{d}}{\mathrm{d} \tau} \nabla^{2} \frac{K}{\widehat{\rho} \tau_{\mathrm{d}}} \mathrm{d} \tau \\
& +18\left\{\left.\frac{K(t)}{\widehat{\rho} \tau_{\mathrm{d}}}\right|_{-\infty}\left[\boldsymbol{w}-\frac{D^{2}}{24} \nabla^{2} \boldsymbol{u}\right]_{-\infty}+\left.\frac{D^{2}}{24} \boldsymbol{w}_{-\infty} \nabla^{2} \frac{K(t)}{\widehat{\rho} \tau_{\mathrm{d}}}\right|_{-\infty}\right\} \\
& -\frac{3}{2} D^{2} \int_{-\infty}^{t_{0}} \frac{\mathrm{d}}{\mathrm{d} \tau}\left(\boldsymbol{\nabla} \frac{K}{\widehat{\rho} \tau_{\mathrm{d}}}\right) \cdot \overline{\overline{\nabla \boldsymbol{u}}}-\boldsymbol{\omega}_{\mathrm{r}} \times \frac{\mathrm{d}}{\mathrm{d} \tau} \nabla \frac{K}{\widehat{\rho} \tau_{\mathrm{d}}} \mathrm{d} \tau \\
& -\frac{3}{2} D^{2}\left\{\left.\nabla \frac{K(t)}{\widehat{\rho} \tau_{\mathrm{d}}}\right|_{-\infty} \cdot \overline{\overline{\nabla \boldsymbol{u}}}_{-\infty}-\boldsymbol{\omega}_{\mathrm{r},-\infty} \times\left.\nabla \frac{K(t)}{\widehat{\rho} \tau_{\mathrm{d}}}\right|_{-\infty}\right\} .
\end{aligned}
$$


The kernel at $\tau=-\infty, K_{-\infty}(t)=0$, therefore

$$
\begin{aligned}
\boldsymbol{f}_{\mathrm{H}, \tau<t_{0}} \equiv 18 \int_{-\infty}^{t_{0}}\left[\boldsymbol{w}-\frac{D^{2}}{24} \nabla^{2} \boldsymbol{u}\right] \frac{\mathrm{d}}{\mathrm{d} \tau} \frac{K}{\widehat{\rho} \tau_{\mathrm{d}}}+\frac{D^{2}}{24} \boldsymbol{w} \frac{\mathrm{d}}{\mathrm{d} \tau} \nabla^{2} \frac{K}{\widehat{\rho} \tau_{\mathrm{d}}} \mathrm{d} \tau \\
-\frac{3}{2} D^{2} \int_{-\infty}^{t_{0}} \frac{\mathrm{d}}{\mathrm{d} \tau}\left(\boldsymbol{\nabla} \frac{K}{\widehat{\rho} \tau_{\mathrm{d}}}\right) \cdot \boldsymbol{\nabla} \boldsymbol{u}-\boldsymbol{\omega}_{\mathrm{p}} \times \frac{\mathrm{d}}{\mathrm{d} \tau} \nabla \frac{K}{\widehat{\rho} \tau_{\mathrm{d}}} \mathrm{d} \tau
\end{aligned}
$$

Moreover, $\boldsymbol{f}_{\mathrm{H}, \tau<t_{0}}=0$ when the motion is started impulsively at $t=t_{0}$. This implies that, in order to have impulsively started motion, for $\tau<t_{0}$ the particle motion and flow velocity have to satisfy

$$
\boldsymbol{w}_{\tau<t_{0}}=\frac{D}{24} \nabla^{2} \boldsymbol{u}_{\tau<t_{0}}
$$

and either

$$
\boldsymbol{\nabla}\left(\frac{K}{\widehat{\rho} \tau_{\mathrm{d}}}\right)_{\tau<t_{0}} \quad \text { or } \quad \boldsymbol{\omega}_{\mathrm{r}, \tau<t_{0}}=\mathbf{0} \quad \text { and } \quad \overline{\bar{\nabla}}_{\tau<t_{0}}=\mathbf{0},
$$

or

$$
\frac{\mathrm{d}}{\mathrm{d} \tau}\left(\nabla \frac{K(t)}{\widehat{\rho} \tau_{\mathrm{d}}}\right)_{\tau<t_{0}} \cdot \overline{\overline{\nabla \boldsymbol{u}}}_{\tau<t_{0}}=\boldsymbol{\omega}_{\mathrm{r}, \tau<t_{0}} \times \frac{\mathrm{d}}{\mathrm{d} \tau}\left(\boldsymbol{\nabla} \frac{K(t)}{\widehat{\rho} \tau_{\mathrm{d}}}\right)_{\tau<t_{0}} .
$$

If impulsively started motion cannot be assumed, the history force can be approximated using $\boldsymbol{f}_{\mathrm{H}, \tau<t_{0}} \approx 0$ if $T_{\mathrm{H}, \tau<t_{0}} \gg 1$, and as a consequence, $K$ and its time derivative are very small, or if for $\tau<t_{0}$, the particle motion and flow velocity satisfy

$$
\left\|\boldsymbol{w}_{\tau<t_{0}}-\frac{D}{24} \nabla^{2} \boldsymbol{u}_{\tau<t_{0}}\right\| \ll 1,
$$

and

$$
\left\|\frac{\mathrm{d}}{\mathrm{d} \tau}\left(\boldsymbol{\nabla} \frac{K(t)}{\widehat{\rho} \tau_{\mathrm{d}}}\right)_{\tau<t_{0}} \cdot \overline{\overline{\nabla \boldsymbol{u}}}_{\tau<t_{0}}-\boldsymbol{\omega}_{\mathrm{r}, \tau<t_{0}} \times \frac{\mathrm{d}}{\mathrm{d} \tau}\left(\boldsymbol{\nabla} \frac{K(t)}{\widehat{\rho} \tau_{\mathrm{d}}}\right)_{\tau<t_{0}}\right\| \ll 1 .
$$

\subsection{First approximation for force and torque}

In preceding sections, the different forces have been derived up to second order accuracy. Generally, only a first order approximation of the force is used and 
higher order terms are neglected. Furthermore, constant fluid properties are assumed. The resulting approximation for the force and torque on a particle with diameter $D$ are

$$
\begin{array}{r}
\widehat{\rho} \frac{\boldsymbol{F}}{m}=(\widehat{\rho}-1) \boldsymbol{f}+\left(1+C_{\mathrm{AM}}\right) \frac{\mathrm{D} \boldsymbol{u}}{\mathrm{D} t}-C_{\mathrm{AM}} \frac{\mathrm{d} \boldsymbol{v}}{\mathrm{d} t} \\
+\left(f_{\mathrm{L}, \mathrm{S}} \boldsymbol{\omega}_{\mathrm{c}}+f_{\mathrm{L}, \mathrm{M}} \boldsymbol{\omega}_{\mathrm{r}}\right) \times \boldsymbol{w}-18 \frac{f_{\mathrm{D}}}{\tau_{\mathrm{d}}}(\mathrm{Re}) \boldsymbol{w} \\
-\frac{18}{\tau_{\mathrm{d}}} \int_{t_{0}}^{t} K(\tau, t) \frac{\mathrm{d} \boldsymbol{w}}{\mathrm{d} \tau} \mathrm{d} \tau-\frac{18}{\tau_{\mathrm{d}}} K_{0}(t) \boldsymbol{w}_{0},
\end{array}
$$

and

$$
\frac{\boldsymbol{T}_{\boldsymbol{\omega}_{\mathrm{r}}}}{I_{\mathrm{p}}}=-\frac{120.6}{\pi \widehat{\rho} \tau_{\mathrm{d}}}\left(1+0.1005 \sqrt{\tau_{\mathrm{d}}\left\|\boldsymbol{\omega}_{\mathrm{r}}\right\|}\right) \boldsymbol{\omega}_{\mathrm{r}},
$$

respectively.

In the present research, this first approximation of the force will be used to obtain predictions for particle trajectories. Furthermore, the lift forces will be neglected in most cases, while the drag and history force will be investigated in more detail. For the case of swirling flow, the influence of the Saffman lift will be investigated for an example case in order to show that the influence of the lift is minor. It is assumed that, for that case, the influence of the Magnus lift will be smaller than that of the Saffman lift, as the relative rotation is expected to be smaller than that of the rotation of the flow field. Furthermore, as particle rotation has only influence on the Magnus lift, the equation for particle rotation is not required for the prediction of the particle movement in this research. 


\section{Chapter 4}

\section{NUMERICAL TREATMENT}

In the preceding chapters, the equations for particle motion, as well as the approximation of the force determining that motion were derived. Analytic methods can be used to predict particle motion for simple cases, such as a simple analytical flow field with the assumption of Stokes flow for the drag and for the Basset history force. However, for increased complexity of the force on the particle, even for analytic flow fields, it is not possible to obtain an analytical solution for the particle motion. For instance, predicting the terminal velocity for a settling particle requires an iterative approach due to the non-linear behaviour of the drag factor. Therefore, numerical treatment of the equations of motion is required. This requires discretization of the differential equation as well as a numerical integration for the history term. First, discretization of the differential equation will be presented. Subsequently, numerical evaluation of the integral in the history force will be described. Finally, the incorporation of the discretized integral in the discretized differential equation will be presented. 


\subsection{Discretized equation of motion}

The equation of motion for position and velocity to be discretized can be written as

$$
\frac{\mathrm{d}}{\mathrm{d} t}\left(\begin{array}{l}
\boldsymbol{x} \\
\boldsymbol{v}
\end{array}\right)=\left(\begin{array}{c}
\boldsymbol{v}(t) \\
\boldsymbol{a}(\boldsymbol{x}(t), \boldsymbol{v}(t), t)
\end{array}\right),
$$

with $\boldsymbol{a}$ the acceleration vector. Using the approximation for the force given in equation (3.89a), the particle acceleration can be defined as

$$
\begin{aligned}
&\left(\widehat{\rho}+C_{\mathrm{AM}}\right) \boldsymbol{a} \equiv(\widehat{\rho}-1) \boldsymbol{f}+\left(1+C_{\mathrm{AM}}\right) \frac{\mathrm{D} \boldsymbol{u}}{\mathrm{D} t} \\
&+\left(f_{\mathrm{L}, \mathrm{S}} \boldsymbol{\omega}_{\mathrm{c}}+f_{\mathrm{L}, \mathrm{M}} \boldsymbol{\omega}_{\mathrm{r}}\right) \times \boldsymbol{w}-18 \frac{f_{\mathrm{D}}}{\tau_{\mathrm{d}}} \boldsymbol{w} \\
&-\frac{18}{\tau_{\mathrm{d}}} \int_{t_{0}}^{t} K(\tau, t) \frac{\mathrm{d} \boldsymbol{w}}{\mathrm{d} \tau} \mathrm{d} \tau-\frac{18}{\tau_{\mathrm{d}}} K_{0}(t) \boldsymbol{w}_{0} .
\end{aligned}
$$

Numerical evaluation of the integral will be presented in next section. However, assuming some discretization for the integral, the differential equations can be discretized. In this research we consider discretization with a Crank-Nicolson method [14]. For such a method the general formulation for particle location and particle velocity at time $t_{n+1}$ is found as

$$
\left(\begin{array}{l}
\boldsymbol{x}_{n+1} \\
\boldsymbol{v}_{n+1}
\end{array}\right)-\left(\begin{array}{l}
\boldsymbol{x}_{n} \\
\boldsymbol{v}_{n}
\end{array}\right)=\frac{t_{n+1}-t_{n}}{2}\left[\left(\begin{array}{c}
\boldsymbol{v}_{n} \\
\boldsymbol{a}_{n}
\end{array}\right)+\left(\begin{array}{c}
\boldsymbol{v}_{n+1} \\
\boldsymbol{a}_{n+1}
\end{array}\right)\right],
$$

where

$$
\begin{gathered}
\boldsymbol{x}_{n} \equiv \boldsymbol{x}\left(t_{n}\right), \\
\boldsymbol{v}_{n} \equiv \boldsymbol{v}\left(t_{n}\right), \\
\boldsymbol{a}_{n} \equiv \boldsymbol{a}\left(\boldsymbol{x}_{n}, \boldsymbol{v}_{n} ; t_{n}\right) .
\end{gathered}
$$

Prediction of the particle position and velocity with this implicit second order method involves a system of non-linear algebraic equations, and requires an iterative solution method. For obtaining an approximation to the solution, methods such as Newton iteration, Gauß-Seidel or Jacobi iteration are equal in performance in this case. It has to be noted that for the Gauß-Seidel method the kernel in the integral of the history force changes every iteration. Calculating this integral only after all velocities have been updated makes computational cost comparable to that of the other methods, without significant reduction of convergence speed. 


\subsection{Numerical integration for the history force}

The properties of the integral in the expression for the history force are a challenge in numerical integration. First of all, the kernel depends on the upper bound $t$ of the integral. As a consequence the integral evaluated at an earlier time-step cannot be reused for the new time-step, resulting in increasingly demanding calculations. Second, the kernel is singular at this upper bound $t$, negating the use of standard integration schemes. Moreover, the lower bound is minus infinity, while the history is known only from some time $t_{0}$. In the preceding chapter treatment of this lower bound of minus infinity has been presented. Finally, in case of the kernel accounting for effects of non-Stokesian flow, the kernel increases the complexity of the integral significantly.

\subsubsection{The general history kernel}

Treatment of the history integral will be derived for the general form of the kernel following van Eijkeren \& Hoeijmakers (2013)[20]. Subsequently, the resulting equation for the Basset kernel, for constant time-step, will be presented as a special case of this general kernel. The general form of the kernel in the history force, from which all presented kernels such as the basset kernel and the more general kernel presented in equation (3.78), can be derived is

$$
K(\tau, t)=\frac{a(\tau, t)}{\left\{b(\tau, t)[T(\tau, t)]^{\frac{1}{4}}+c(\tau, t) T(\tau, t)\right\}^{2}},
$$

where $a, b$ and $c$ depend on the kernel used. The generalized integral to be treated numerically is

$$
\int_{t_{0}}^{t} K \frac{\mathrm{d} \boldsymbol{w}}{\mathrm{d} \tau} \mathrm{d} \tau
$$

First the lowest order singularity in terms of $T$ will be split from the kernel. This results in

$$
\int_{t_{0}}^{t} K \frac{\mathrm{d} \boldsymbol{w}}{\mathrm{d} \tau} \mathrm{d} \tau=\int_{t_{0}}^{t} \frac{\boldsymbol{h}}{\sqrt{T}} \mathrm{~d} \tau
$$

where

$$
\boldsymbol{h} \equiv \sqrt{T} K \frac{\mathrm{d} \boldsymbol{w}}{\mathrm{d} \tau}=\frac{a}{\left[b+c T^{\frac{3}{4}}\right]^{2}} \frac{\mathrm{d} \boldsymbol{w}}{\mathrm{d} \tau}
$$


Subsequently, a change of integraion variables from $\tau$ to $T$ is applied. The integral then becomes

$$
\int_{t_{0}}^{t} K \frac{\mathrm{d} \boldsymbol{w}}{\mathrm{d} \tau} \mathrm{d} \tau=\int_{T_{0}}^{T_{t}} \frac{\boldsymbol{h}}{\sqrt{T}}\left[\frac{\mathrm{d} T}{\mathrm{~d} \tau}\right]^{-1} \mathrm{~d} T,
$$

where $T_{t}=0$. The derivative of the history time with respect to $\tau$ is

$$
\frac{\mathrm{d} T}{\mathrm{~d} \tau}=-\frac{1}{\tau_{\mathrm{d}}} \frac{\operatorname{Re}(\tau)}{\operatorname{Re}(t)},
$$

which reduces to $-\frac{1}{\tau_{\mathrm{d}}}$ in case of the limit $\mathrm{Re} \rightarrow 0$ for the Basset history force.

Partial integration in equation (4.9) now leads to

$$
\int_{t_{0}}^{t} K \frac{\mathrm{d} \boldsymbol{w}}{\mathrm{d} \tau} \mathrm{d} \tau=\left.2 \sqrt{T}\left[\frac{\mathrm{d} T}{\mathrm{~d} \tau}\right]^{-1} \boldsymbol{h}\right|_{T_{0}} ^{0}-2 \int_{T_{0}}^{0} \sqrt{T} \frac{\mathrm{d}}{\mathrm{d} T}\left(\left[\frac{\mathrm{d} T}{\mathrm{~d} \tau}\right]^{-1} \boldsymbol{h}\right) \mathrm{d} T .
$$

The derivative with respect to $T$ will be considered piecewise constant, which results in an approximation of a sufficient order to retain the order of accuracy for the discretized equation of motion. The quadrature of the integral with upper bound $t_{n}$ becomes

$$
\begin{aligned}
\int_{t_{0}}^{t_{n}} K \frac{\mathrm{d} \boldsymbol{w}}{\mathrm{d} \tau} \mathrm{d} \tau \approx & -2 \sqrt{T_{0}}\left[\frac{\mathrm{d} T}{\mathrm{~d} \tau}\right]_{0}^{-1} \boldsymbol{h}_{0} \\
& +\frac{4}{3} \sum_{i=0}^{n-1}\left(T_{i}^{\frac{3}{2}}-T_{i+1}^{\frac{3}{2}}\right) \frac{\left[\frac{\mathrm{d} T}{\mathrm{~d} \tau}\right]_{i+1}^{-1} \boldsymbol{h}_{i+1}-\left[\frac{\mathrm{d} T}{\mathrm{~d} \tau}\right]_{i}^{-1} \boldsymbol{h}_{i}}{T_{i+1}-T_{i}}
\end{aligned}
$$

\subsubsection{Basset kernel with constant time-step}

A special case of the quadrature of the integral in the history force is the quadrature of the integral with the Basset kernel using a constant time-step $\Delta t$. The resulting discretized integral is

$$
\begin{aligned}
\int_{t_{0}}^{t_{n}} K_{B} & \left.\frac{\mathrm{d} \boldsymbol{w}}{\mathrm{d} \tau} \mathrm{d} \tau \approx\left\{\sqrt{n}+\frac{2}{3}\left[n^{\frac{3}{2}}-(n-1)^{\frac{3}{2}}\right]\right\} \sqrt{\frac{\tau_{\mathrm{d}} \Delta t}{\pi}} \frac{\mathrm{d} \boldsymbol{w}}{\mathrm{d} t}\right|_{0} \\
+ & \frac{2}{3} \sqrt{\frac{\tau_{\mathrm{d}} \Delta t}{\pi}}\left\{\left.\frac{\mathrm{d} \boldsymbol{w}}{\mathrm{d} t}\right|_{n}+\left.\sum_{i=1}^{n-1}\left[\left(j_{i}+1\right)^{\frac{3}{2}}-2 j_{i}^{\frac{3}{2}}+\left(j_{i}-1\right)^{\frac{3}{2}}\right] \frac{\mathrm{d} \boldsymbol{w}}{\mathrm{d} t}\right|_{i}\right\}
\end{aligned}
$$




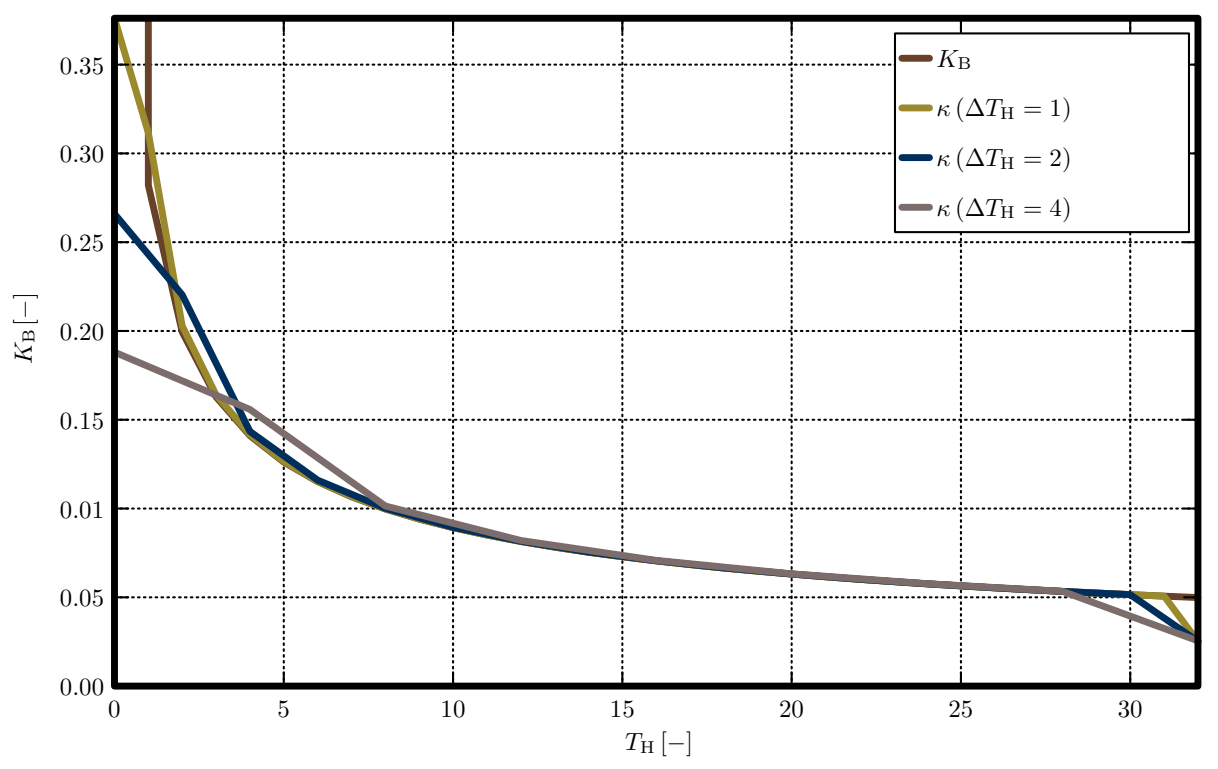

Figure 4.1: Comparison of exact and discrete Basset kernels for various stepsizes $\Delta T_{\mathrm{H}}$ in Basset history time, with $t_{n}=32 \tau_{d}$ and $n=\frac{t_{n}}{\Delta T}$.

with $j_{i} \equiv n-i$.

In this case, the a discretized kernel $\kappa_{j_{i}}$ can be defined for $0<j<n$, where $n$ is the number of time-steps required in the calculation. This discrete kernel is

$$
\kappa_{j_{i}}= \begin{cases}\frac{4}{3} K_{\mathrm{B}}(0, \Delta t) & j_{i}=0, \\ \frac{4}{3}\left[\left(j_{i}+1\right)^{\frac{3}{2}}-2 j_{i}^{\frac{3}{2}}+\left(j_{i}-1\right)^{\frac{3}{2}}\right] K_{\mathrm{B}}(0, \Delta t) & 0<j_{i}<n, \\ \left\{2 \sqrt{n}+\frac{4}{3}\left[(n-1)^{\frac{3}{2}}-n^{\frac{3}{2}}\right]\right\} K_{\mathrm{B}}(0, \Delta t) & j_{i}=n,\end{cases}
$$

and the discretized history integral then becomes

$$
\left.\int_{t_{0}}^{t_{n}} K_{B} \frac{\mathrm{d} \boldsymbol{w}}{\mathrm{d} \tau} \mathrm{d} \tau \approx \sum_{i=0}^{n} \kappa_{j} \frac{\mathrm{d} \boldsymbol{w}}{\mathrm{d} t}\right|_{i} \Delta t .
$$

Figure 4.1 shows the exact and discretized Basset kernel for various timesteps. It can be observed that the discretized kernel is very close to the exact 
kernel for most of the region except for $t_{n}, t_{n-1}$ and $t_{0}$.

\subsection{Incorporation of the discrete history integral}

Often, the history force is calculated using the velocity history $[5,17]$. Using the approach presented in the preceding section, the relative acceleration history of the particle is used instead. The relative acceleration of a particle is

$$
\frac{\mathrm{d} \boldsymbol{w}}{\mathrm{d} t}=\boldsymbol{a}-\frac{\mathrm{D} \boldsymbol{u}}{\mathrm{D} t}-\boldsymbol{w} \cdot \boldsymbol{\nabla} \boldsymbol{u} .
$$

Therefore, the acceleration at time-step $n$ becomes

$$
\begin{gathered}
\left(\widehat{\rho}+C_{\mathrm{AM}}+24 \sqrt{T}_{n-1} \sqrt{T}_{n} K_{n}\right) \boldsymbol{a}_{n} \equiv 24 \sqrt{T}_{n-1} \sqrt{T}_{n} K_{n} \boldsymbol{w}_{n} \cdot \nabla \boldsymbol{u}_{n} \\
+\left(f_{\mathrm{L}, \mathrm{S}, n} \boldsymbol{\omega}_{\mathrm{c}, n}+f_{\mathrm{L}, \mathrm{M}, n} \boldsymbol{\omega}_{\mathrm{r}, n}\right) \times \boldsymbol{w}_{n}-18 \frac{f_{\mathrm{D}, n}}{\tau_{\mathrm{d}}} \boldsymbol{w}_{n}-\frac{18}{\tau_{\mathrm{d}}} K_{0}(t) \boldsymbol{w}_{0} \\
+\frac{24}{\tau_{\mathrm{d}}}\left\{\frac{3}{2} \sqrt{T}_{0}-\frac{T_{1}^{\frac{3}{2}}-T_{0}^{\frac{3}{2}}}{T_{1}-T_{0}}\right\}\left[\frac{\mathrm{d} T}{\mathrm{~d} \tau}\right]_{0}^{-1} \sqrt{T}_{0} K_{0} \boldsymbol{\alpha}_{0} \\
-\frac{24}{\tau_{\mathrm{d}}} \sum_{i=0}^{n-1}\left(\frac{T_{i+1}^{\frac{3}{2}}-T_{i}^{\frac{3}{2}}}{T_{i+1}-T_{i}}-\frac{T_{i}^{\frac{3}{2}}-T_{i-1}^{\frac{3}{2}}}{T_{i}-T_{i-1}}\right)\left[\frac{\mathrm{d} T}{\mathrm{~d} \tau}\right]_{i}^{-1} \sqrt{T}_{i} K_{i} \boldsymbol{\alpha}_{i} \\
+(\widehat{\rho}-1) \boldsymbol{f}_{n}+\left.\left(1+C_{\mathrm{AM}}+24 \sqrt{T}_{n-1} \sqrt{T}_{n} K_{n}\right) \frac{\mathrm{D} \boldsymbol{u}}{\mathrm{D} t}\right|_{n},
\end{gathered}
$$

with the relative acceleration history

$$
\boldsymbol{\alpha}_{i} \equiv \boldsymbol{a}_{i}-\left.\frac{\mathrm{D} \boldsymbol{u}}{\mathrm{D} t}\right|_{i}-\boldsymbol{w}_{i} \cdot \boldsymbol{\nabla} \boldsymbol{u}_{i} .
$$

The history force in the presented discretized formulation results in a similar effect as the added mass effect, combined with a force depending on the time-history of the particle. The time history of $\boldsymbol{\alpha}_{i}$, as well as $T_{i}$ and $\mathrm{Re}_{i}$ are required. The history time $T_{i}$ can be obtained using standard numerical integration schemes such as the trapezoidal rule, or Simpson's rule for numerical evaluation of integrals. Moreover, the result of $\operatorname{Re}_{n} T_{n-1}$ can be used to obtain $\operatorname{Re}_{n} T_{n}$. On the other hand, $\operatorname{Re}_{i}$ can be reconstructed from $\operatorname{Re}_{n} T_{i-1}$ and $\operatorname{Re}_{n} T_{i}$. This implies that, in order to calculate the history force, only $\boldsymbol{\alpha}_{i}$ and $\operatorname{Re}_{n} T_{i}$ need to be stored. The particle acceleration, defined by equation (4.17), is used to predict particle trajectories for settling spheres and droplets in a swirling flow field. 


\section{CHAPTER 5}

\section{SETTLING SPHERE}

The discretized equations of motion are applied to the case of settling spheres. Experimental data for the time-dependent settling velocity of spheres in water have been obtained by Mordant \& Pinton (2000) [32]. These experiments have been used as a basis to validate the expression for the kernel in the history force. In this chapter, particle trajectories are predicted using the discretized equations of motion and compared to the experimental results. The results have been presented in van Eijkeren \& Hoeijmakers (2013) [20].

\subsection{Flow and particle attributes}

Flow and particle properties for the calculation of the particle trajectories have been chosen to match the experiments. However, the reported densities of glass and iron spheres vary much $[32,20]$. Moreover, reported Reynolds numbers do not agree with reported diameters and velocities. It is likely that reported densities are a result from measured diameter and mass, and densities are actually equal for spheres of equal material. Therefore, for the present numerical simulations it has been assumed that each material has an average density. This density has been obtained as the volume averaged density of all reported spheres of a specific material

$$
\rho_{\text {Material }}=\frac{\sum_{i} \rho_{i} V_{i}}{\sum_{i} V_{i}} .
$$


Furthermore, the diameter of the spheres has been adapted in such a way that the terminal velocity $\left\|\boldsymbol{v}_{\mathrm{t}}\right\|$ calculated using the correlation by Cheng (2009), equation (3.66), matches with the one measured in the experiments. The terminal velocity for a particle is obtained by solving

$$
18 \frac{f_{\mathrm{D}}}{\tau_{\mathrm{d}}} \boldsymbol{v}=(\hat{\rho}-1) \boldsymbol{g} .
$$

The diameters of the spheres that follow from matching the predicted and reported terminal velocity, are presented in table 5.2. These diameters are close to the reported diameters for the experiment. Moreover, considering the significance of the experimental data, the diameters can be considered the same. However, the terminal velocities can change significantly when using the diameter reproted in the description of the experiment, as the diffusive time-scale is a function of the square of the diameter. Moreover, the drag factor depends on the diameter as well. Mordant \& Pinton used a glass sphere for experiment \#1 while they used steel spheres for experiments \#4 to \#9. The volume averaged densities as well as the fluid properties of water approximating the experimental conditions are presented in table 5.1.

\subsection{Results and discussion}

The predicted terminal velocity as a function of time for the cases presented in table 5.2 is obtained performing simulations without history force, with Basset history force and with the history force proposed in the present study. The results are shown in figures 5.1 to 5.8. In the figures, the reported experimental data [32] is also shown. The reported experimental data is reported to be an average of multiple experiments using the same set-up and conditions. It can be observed that the acceleration of the particle is over-predicted significantly in the simulation performed without taking the history force into account. It can also be observed that in the simulations performed using the Basset kernel, the acceleration is significantly under-predicted for cases number 1, and numbers 4 to 6 . However, for case number 7 the initial acceleration is close to the experimental data. Only in the latter part of the acceleration, the Basset history force remains too large. For cases number 8 and number 9 the initial acceleration is even over predicted by the simulation with the Basset history force, while the terminal velocity is still not reached soon enough.

The history force was also experimentally determined by Mordant \& Pinton [32] for the iron sphere of case number 5. The acceleration of the particle was 


\begin{tabular}{|c|c|c|c|c|}
\hline $\begin{array}{c}\rho_{\mathrm{H}_{2} \mathrm{O} @ 25^{\circ} \mathrm{C}} \\
{\left[\mathrm{kg} \mathrm{m}^{-3}\right]}\end{array}$ & $\begin{array}{c}\mu_{\mathrm{H}_{2} \mathrm{O} @ 25^{\circ} \mathrm{C}} \\
{\left[\mathrm{kg} \mathrm{m}^{-1} \mathrm{~s}^{-2}\right]}\end{array}$ & {$\left[\begin{array}{c}g \\
{\left[\mathrm{~ms}^{-2}\right]}\end{array}\right.$} & $\begin{array}{c}\rho_{\text {glass }} \\
{\left[\mathrm{kg} \mathrm{m}^{-3}\right]}\end{array}$ & $\begin{array}{c}\rho_{\text {steel }} \\
{\left[\mathrm{kg} \mathrm{m}^{-3}\right.}\end{array}$ \\
\hline 997.04 & $8.937 \times 10^{-4}$ & 9.807 & 2504.35 & 7742.38 \\
\hline
\end{tabular}

Table 5.1: Fluid properties of water at the experimental condition of $25^{\circ} \mathrm{C}$ and the estimated gravitational constant in Lyon, where the experiment was performed [32]. Volume averaged densities of the spheres used in the simulations.

$\begin{array}{cccccc}\# & \begin{array}{c}\rho_{\exp } \\ {\left[\mathrm{kg} \mathrm{m}^{-3}\right]}\end{array} & \begin{array}{c}D_{\exp } \\ \times 10^{-3}[\mathrm{~m}]\end{array} & \begin{array}{c}D_{\text {sim }} \\ \times 10^{-3}[\mathrm{~m}]\end{array} & \begin{array}{c}\left\|\boldsymbol{v}_{\mathrm{t}}\right\| \\ {\left[\mathrm{m} \mathrm{s}^{-1}\right]}\end{array} & \begin{array}{c}\text { Re } \\ {[-]}\end{array} \\ 1 & 2560 & 0.5 & 0.494866 & 0.0741 & 41 \\ 4 & 7710 & 0.8 & 0.782653 & 0.316 & 280 \\ 5 & 7850 & 1 . & 0.982777 & 0.383 & 430 \\ 6 & 7670 & 2 . & 2.00398 & 0.636 & 1400 \\ 7 & 7800 & 3 . & 3.06843 & 0.813 & 2700 \\ 8 & 7700 & 4 . & 4.34968 & 0.973 & 4300 \\ 9 & 7750 & 6 . & 6.25205 & 0.636 & 7700\end{array}$

Table 5.2: Diameter $D_{\text {sim }}$ used in the numerical simulations [20] as a result of matching the predicted terminal velocity to the reported terminal velocity [32]. Predicted terminal velocity, equation (5.2), is based on the properties reported in table 5.1, and used drag correlation, equation (3.66). 


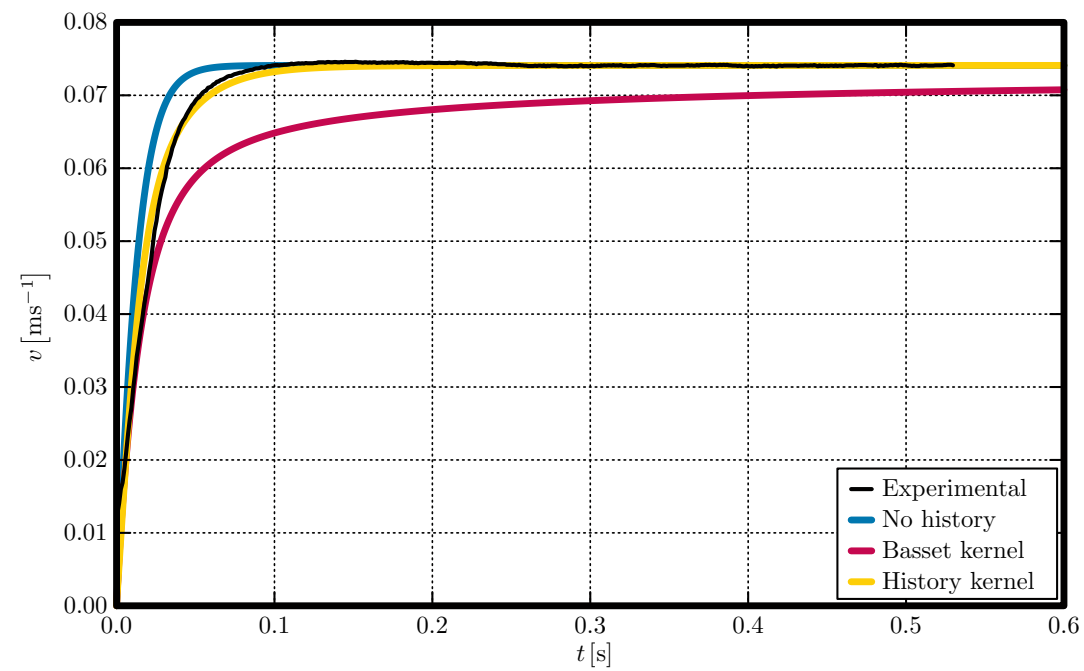

Figure 5.1: Predicted velocity as a function of time for run number 1 compared to experimentally obtained velocity [32]. Glass sphere, $\mathrm{Re}=41$.

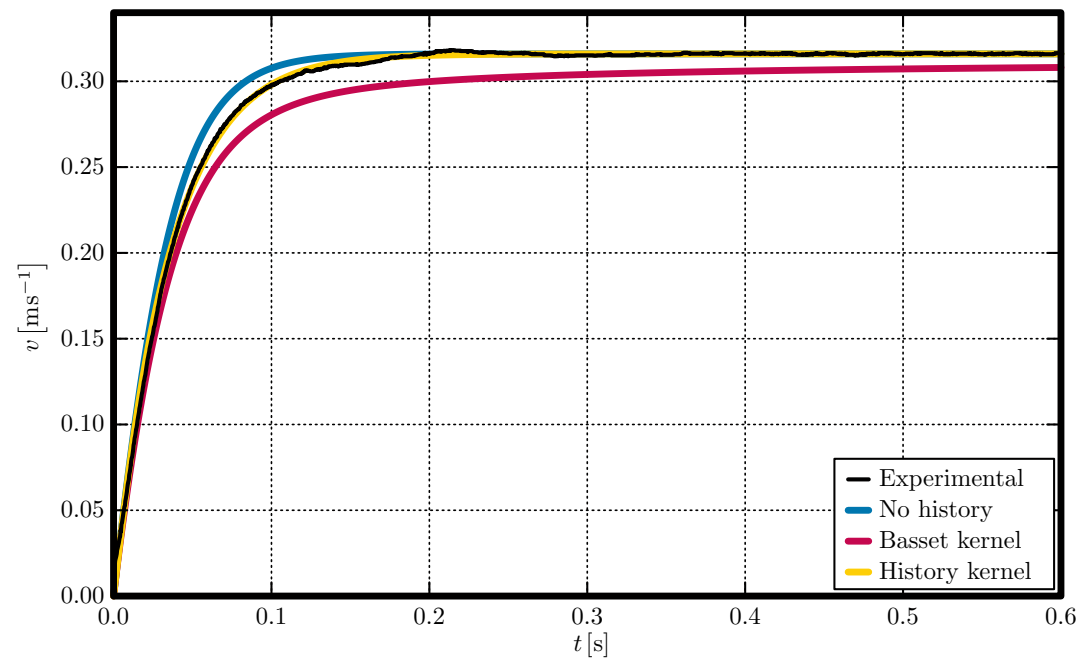

Figure 5.2: Predicted velocity as a function of time for run number 4 compared to experimentally obtained velocity [32]. Iron sphere, $\mathrm{Re}=280$. 


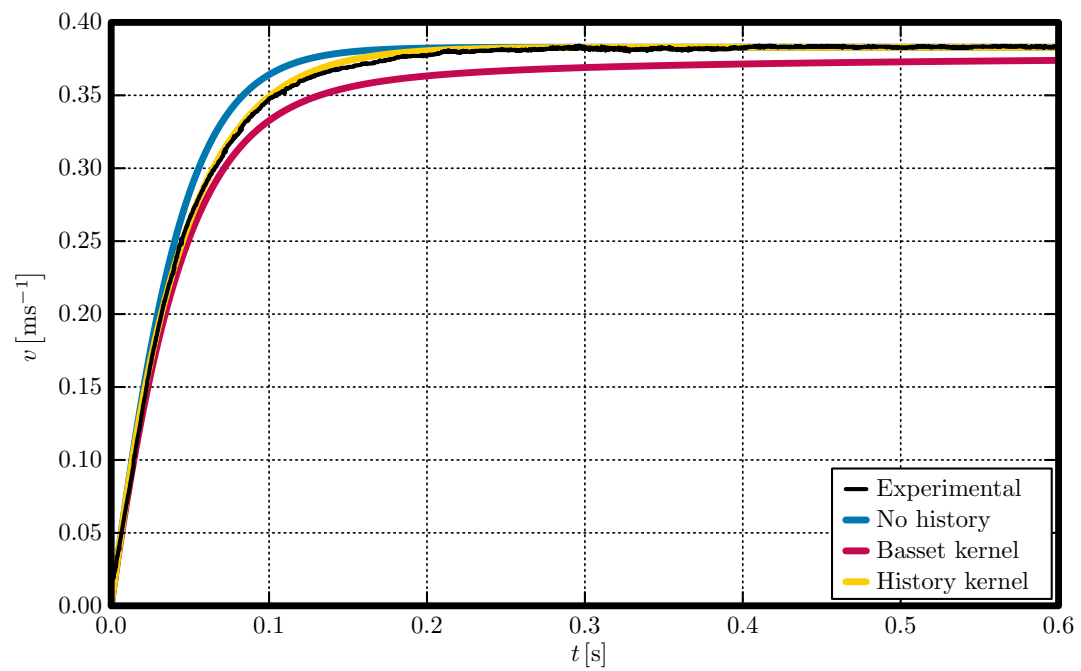

Figure 5.3: Predicted velocity as a function of time for run number 5 compared to experimentally obtained velocity [32]. Iron sphere, $R e=430$.

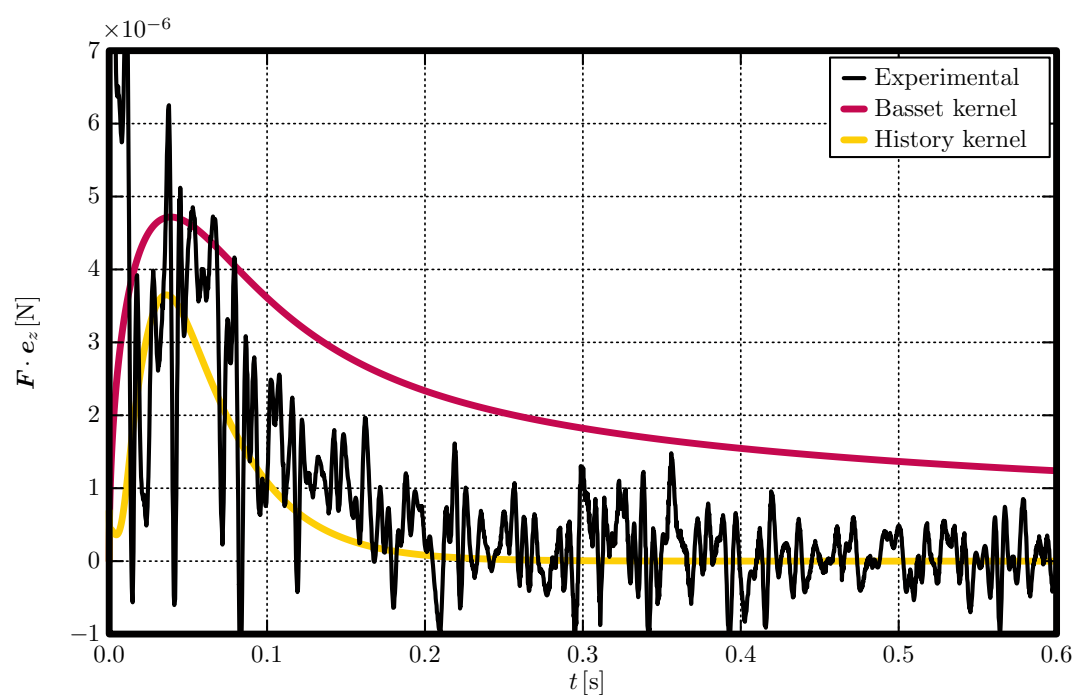

Figure 5.4: The predicted history force for run number 5 compared to the history force derived from the experimental data [32]. Iron sphere, $\operatorname{Re}=430$. 


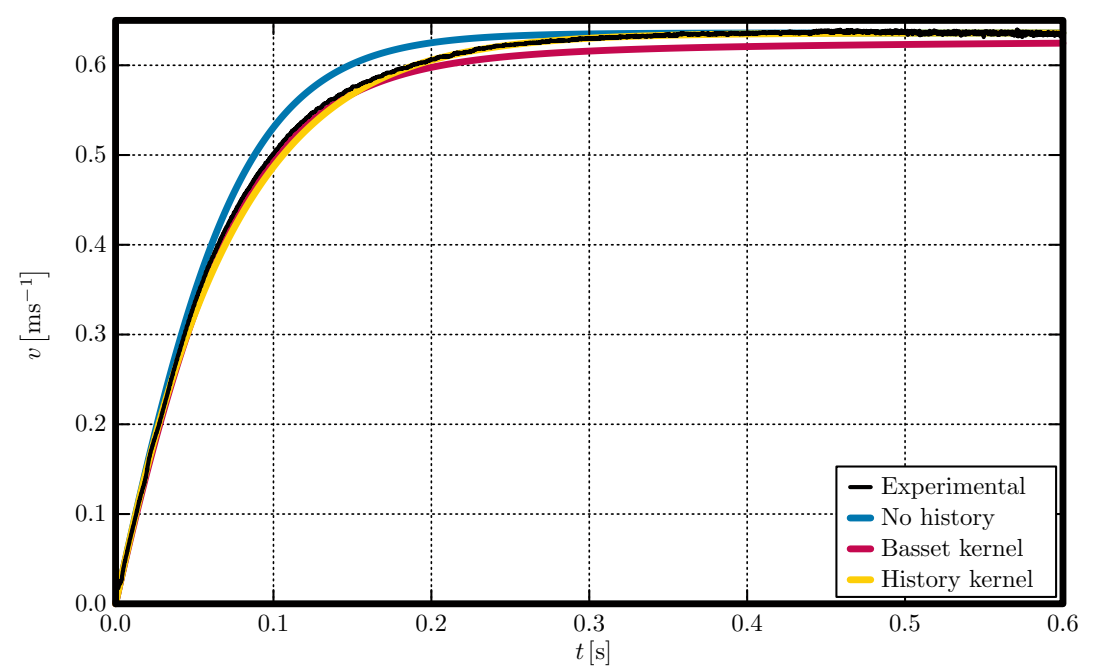

Figure 5.5: Predicted velocity as a function of time for run number 6 compared to experimentally obtained velocity [32]. Iron sphere, $R e=1400$.

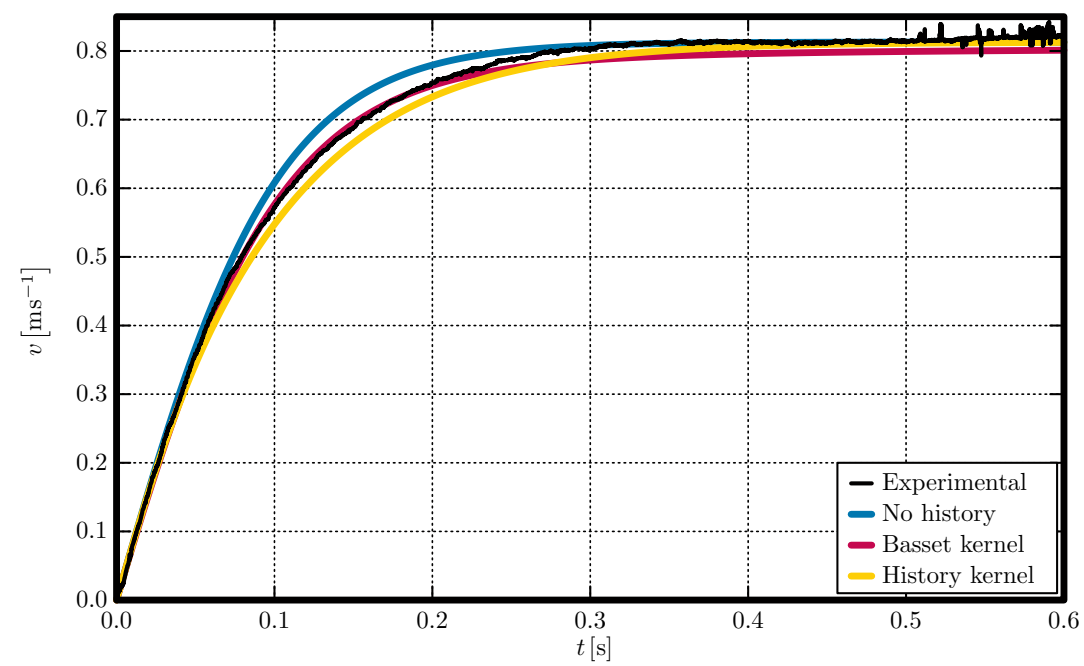

Figure 5.6: Predicted velocity as a function of time for run number 7 compared to experimentally obtained velocity [32]. Iron sphere, $R e=2700$. 


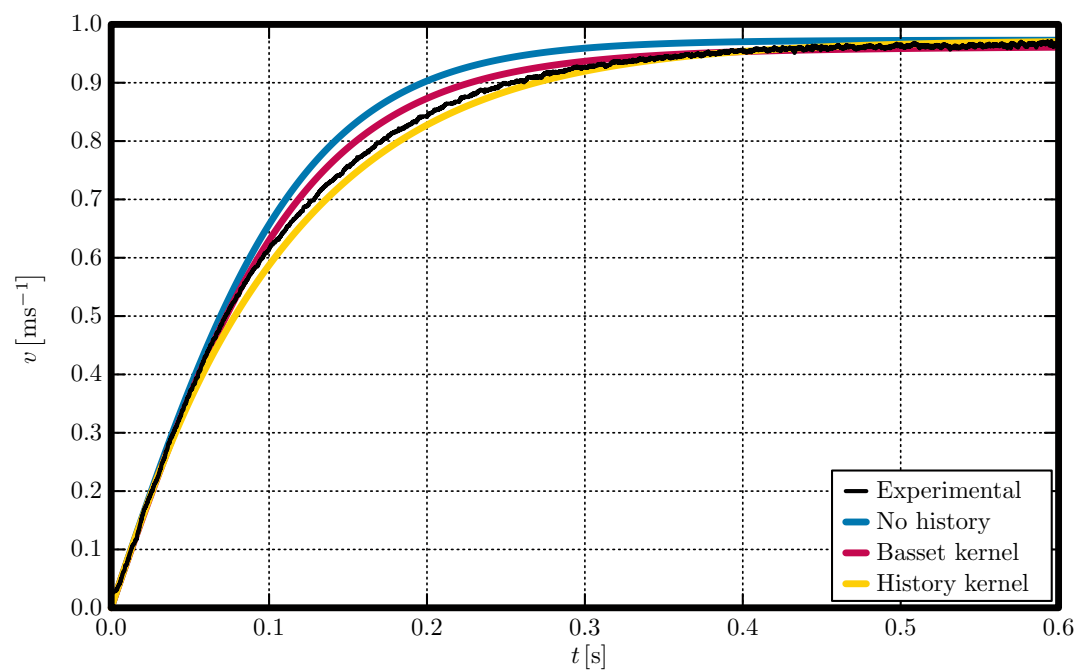

Figure 5.7: Predicted velocity as a function of time for run number 8 compared to experimentally obtained velocity [32]. Iron sphere, $\mathrm{Re}=4300$.

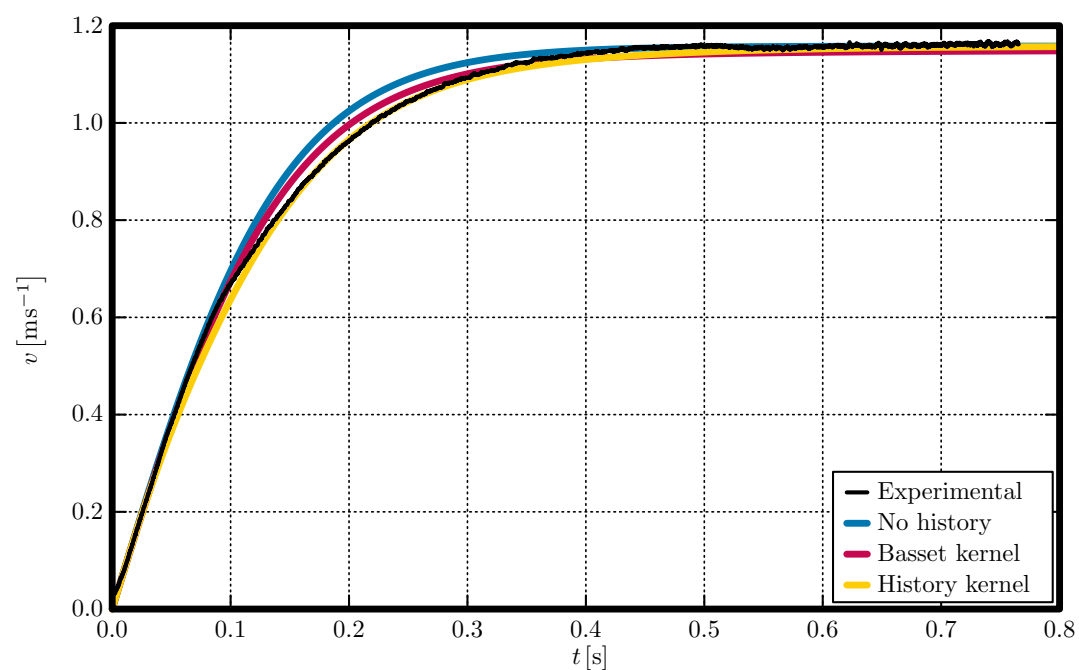

Figure 5.8: Predicted velocity as a function of time for run number 9 compared to experimentally obtained velocity [32]. Iron sphere, $\mathrm{Re}=7700$. 
determined from the velocity measurements leading to the force on the particle. Subsequently, known forces such as the drag force, buoyancy force and added mass force were subtracted from the experimentally determined force. The remaining force contains the history force as well as other effects. However, for the case of a settling sphere, other effects would be minor, resulting in a test case for determining the actual history force. One of the disadvantages for this way of obtaining the history force, is that it depends on the chosen approximations for the drag force and added mass force. The history force presented in figure 5.4 shows that, the Basset force can capture the short term peak in the history force. However, it fails to capture the long term behaviour and does not manage to capture the fast decline in history force that is observed in the experiments.

Using the results for the predictions without history and with Basset history force it can be concluded that the kernel in the history force should indeed decline faster than the Basset kernel. However, the history kernel should also result in a higher initial effect of acceleration for intermediate to high Reynolds numbers. The history kernel proposed in equation (3.78) aims to capture observed behaviour. The kernel declines second order in time for long term behaviour leading to the required faster decline of the kernel. Moreover, as the history time depends on the Reynolds number, the history time increases faster for higher Reynolds numbers. Furthermore, the drag factor and Reynolds number dependency leads to a higher initial effect of acceleration for intermediate Reynolds numbers.

The results of the numerical simulations, presented in figures 5.1 to 5.8 , show that results using the proposed history kernel are close to the experimental data. Runs \#1 and \#4 to \#6 succeed to capture the faster decline of the history kernel. However, in run \#7 the history force is over-predicted leading to smaller accelerations than observed in the experiments. In runs \#8 and \#9 the proposed history kernel captures the increased history force due to effects of the drag factor and Reynolds number. Moreover, comparing the predicted history force to the history force derived from the measurements, it is observed that the proposed history kernel succeeds to capture the rate of decline, although the onset of the decline is slightly earlier. The history force derived from the measurements depends on the chosen approximation for the added mass and drag force, which complicates quantitative comparison. However, qualitatively, predicted and measured results are similar.

It can be concluded that the proposed history kernel, equation (3.78), is a good candidate to use in the approximation of the history force. The kernel can now be used in predictions for particle trajectories in generic flow fields, such as a the flow field in a swirling flow separator. 


\section{Chapter 6}

\section{OIL DROPLET IN SWIRLING FLOW}

For oil-water separation, swirling flow separators are employed for produced water treatment. The flow in such a device is complex, and simulation of such a flow is not trivial. In this chapter, the flow field in a swirling flow separator will be approximated by a simplified macroscopic flow-field consisting of a LambOseen vortex superposed on an axial flow field. This flow field is used as input for the prediction of trajectories of oil-droplets typical for water treatment. Particle trajectories are predicted for several approximations for the drag and history force, and results are discussed. Results show that inertia has a significant influence on particle trajectories. Moreover, results show that the history force has a contribution of the same order of magnitude. Therefore it has to be taken into account when predicting the required dimensions of a swirling flow separator. The discription of the swirling flow field, and the results of particle tracking in such a swirling flow field have been presented in van Eijkeren \& Hoeijmakers (2013) [20].

\subsection{Swirling flow and particle attributes}

\subsubsection{Swirling flow field}

In a swirling flow separator the macroscopic flow field typically consists of a combination of a solid body rotation and that of an inviscid potential flow 
vortex, with an intermediate region in between, which results in the so-called Lamb-Oseen vortex. Moreover, the radial distribution of the axial flow field observed for such a swirling flow is typically a W-shaped profile [37]. In this research the flow field mimics an in-line swirling flow separator with constant radius. Moreover, in this research the axial and azimuthal velocity distributions are considered constant in axial direction. As a result, the radial velocity will be zero. Note that for actual swirling flow separators, the distributions will change due to decay of swirl as well as due to a tapered geometry.

A Lamb-Oseen like vortex, with circulation $\Gamma$ and viscous decay-time $\tau_{\nu}$, for a fluid with kinematic viscosity $\nu$ results in an representative azimuthal velocity distribution. The $\mathrm{W}$-shaped distribution of the axial velocity has a maximum velocity $U$. Moreover, the reverse flow velocity parameter $U_{0}$ and centre velocity parameter $U_{1}$ determine the minimum velocity of the profile and the velocity at the centre of rotation, i.e. the axis of the pipe. The second viscous decay time $\tau_{\nu, 1}$ further defines the $\mathrm{W}$-shaped profile. The fluid velocity field [20] is described by

$$
\left(\begin{array}{l}
u_{r} \\
u_{\theta} \\
u_{z}
\end{array}\right)=\left(\begin{array}{c}
0 \\
\frac{\Gamma}{2 \pi r}\left(1-e^{-\frac{r^{2}}{\tau_{\nu} \nu}}\right) \\
U-U_{0} e^{-\frac{r^{2}}{\tau_{\nu \nu}}}+U_{1} e^{-\frac{r^{2}}{\tau_{\nu, 1^{\nu}}}}
\end{array}\right) .
$$

The parameters $\tau_{\nu}, \tau_{\nu, 1}, U_{0}, U_{1}, U$ and $R$ determine the volume flow in axial direction. This volume flow should be equal to the volume flow in a pipe with radius $R$ and bulk velocity $U_{\infty}$. This implies that for a pipe with radius $R$ and bulk velocity $U_{\infty}$ and with $\tau_{\nu}, \tau_{\nu, 1}, U_{0}$ and $U_{1}$ chosen, the maximum velocity becomes

$$
U=U_{\infty}+U_{0} \frac{\tau_{\nu} \nu}{R^{2}}\left(1-e^{-\frac{R^{2}}{\tau_{\nu} \nu}}\right)-U_{1} \frac{\tau_{\nu, 1} \nu}{R^{2}}\left(1-e^{-\frac{R^{2}}{\tau_{\nu, 1^{\nu}}}}\right)
$$

The parameters, selected to obtain typical flow conditions in a swirling flow separator, including features such as flow reversal, are shown in table 6.1. The resulting velocity profiles as a function of the relative radius $\frac{r}{R}$ are shown in figure 6.1. The axial velocity is shown as fraction of the bulk velocity $U_{\text {ref, } z}=$ $U_{\infty}$, while the azimuthal velocity is shown as fraction of the azimuthal velocity at radius $R$ of an inviscid vortex with the same circulation i.e. $U_{\text {ref, } \theta}=\frac{\Gamma}{2 \pi R}$.

The flow field described by equation (6.1) conserves mass. However, it does not conserves momentum purely by its velocity terms. It is assumed that the pressure gradient and the effects of turbulence contribute to the flow field in such a way that conservation of momentum is conserved. 


$\begin{array}{cccc}\tau_{\nu}[\mathrm{s}] & \Gamma\left[\mathrm{m}^{2} \mathrm{~s}^{-1}\right] & \nu\left[\mathrm{m}^{2} \mathrm{~s}^{-1}\right] & R[\mathrm{~m}] \\ 500 & 2 & 8.92 \times 10^{-7} & 0.05 \\ \tau_{\nu, 1}[\mathrm{~s}] & U_{\infty}\left[\mathrm{m} \mathrm{s}^{-1}\right] & U_{0}\left[\mathrm{~ms}^{-1}\right] & U_{1}\left[\mathrm{~m} \mathrm{~s}^{-1}\right] \\ \frac{250}{3} & 2 & 6 & \frac{7}{2}\end{array}$

Table 6.1: Parameters for the swirling flow field, used to mimic a flow field typical for a swirling flow separator used for produced water treatment.

$$
\begin{array}{cccc}
\rho_{\mathrm{H}_{2} \mathrm{O}}\left[\mathrm{kg} \mathrm{m}^{-3}\right] & \nu_{\mathrm{H}_{2} \mathrm{O}}\left[\mathrm{m}^{2} \mathrm{~s}^{-1}\right] & D[\mathrm{~m}] & \rho_{\text {oil }}\left[\mathrm{kg} \mathrm{m}^{-3}\right] \\
997 & 8.92 \times 10^{-7} & 1 \times 10^{-4} & 800
\end{array}
$$

Table 6.2: Material attributes of the continuous phase and the oil droplet.

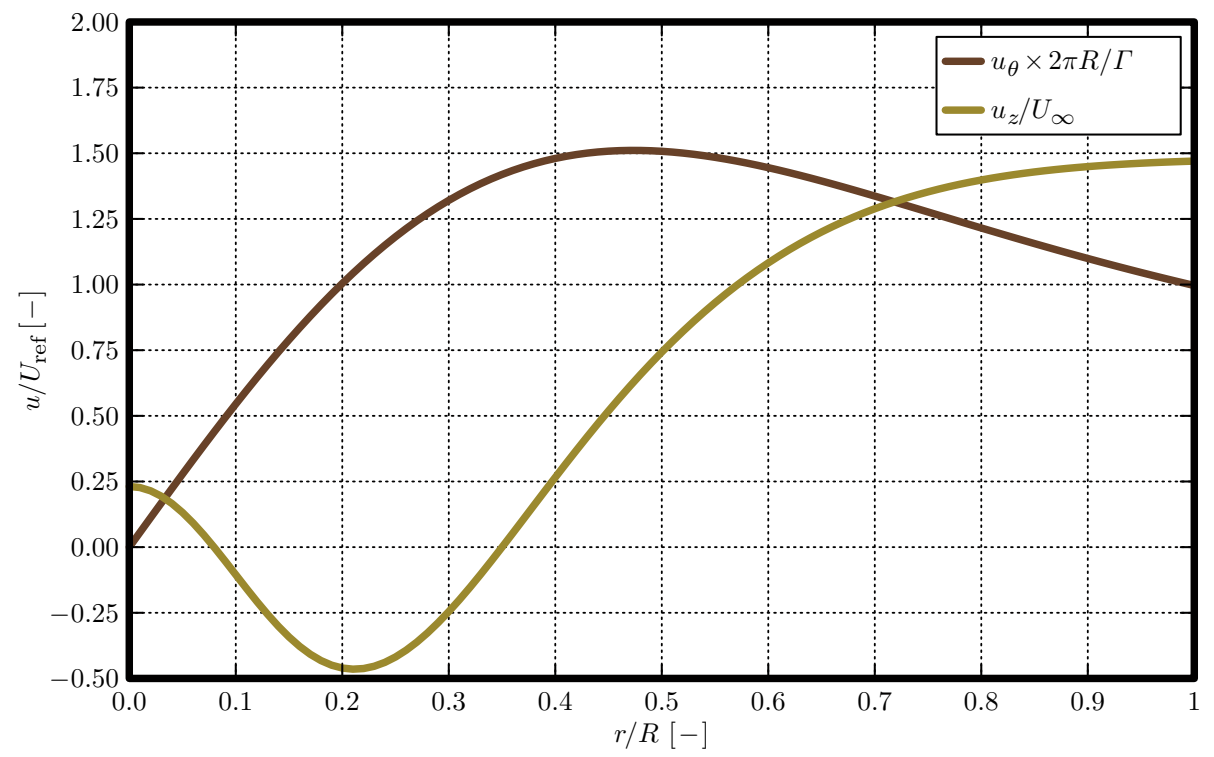

Figure 6.1: The axial $u_{z}$ and azimuthal $u_{\theta}$ components of the velocity field presented in equation (6.1). The reference velocities are the bulk velocity $U_{\infty}$ and the azimuthal velocity at radius $R$ of an inviscid potential flow vortex with circulation $\Gamma$, respectively. 


\subsubsection{Fluid and particle properties}

Numerical simulations have been performed to predict the particle trajectory of an oil droplet in water. The properties of water and oil-droplet, as used in the swirling flow simulation to obtain the force on the droplet, are shown in table 6.2. The material properties represent an oil-droplet of $100[\mu \mathrm{m}]$ in water at room temperature.

\subsection{Results and discussion}

\subsubsection{Non-Stokesian effects, history force and lift}

The trajectory of an oil droplet with the properties given in table 6.2 in the swirling flow field described by equation (6.1) with the parameters given in table 6.1 and with the material properties given in table 6.2. Centrifugal forces will be dominant compared to buoyancy. Therefore, gravitational effects will be neglected. Oil has a high viscosity with respect to water. Moreover, the oil droplet will be very small. Therefore, the oil droplet is considered rigid and of spherical shape.

The influence of non-Stokesian effects is investigated by comparing the results of simulations using Stokes' drag and simulations using the drag factor by Cheng (2009), both without taking into account the history force. Furthermore, the influence of the history force is investigated by performing simulations including the effects of the Basset history force and by including the non-Stokesian history force. The resulting particle trajectories in the $r, z$-plane are shown in figure 6.2. Moreover, for one of the particle trajectories, the trajectory projected on the $x, y$-plane is presented, see figure 6.3. In the figures, the effect of the characteristics of the flow field can be observed. The particle follows the swirling motion of the field, while the stress gradient, primarily caused by centrifugal effects, forces the particle towards the axis of rotation, resulting in a spiraling trajectory. Furthermore, the annular region of reversed flow results in a change in the axial direction of the particle motion. First the particle gradually decreases its axial velocity while in the annular region, the motion is entirely reversed. Finally, the particle enters the central region with positive axial flow velocities and there it travels in positive axial direction again.

From figure 6.2 it can be concluded that only taking into account only the Stokes drag as well as not taking into account history effects, significantly underestimates the axial distance traveled before the droplet enters the central region. Therefore, if a device would have been designed on basis of these results, the 


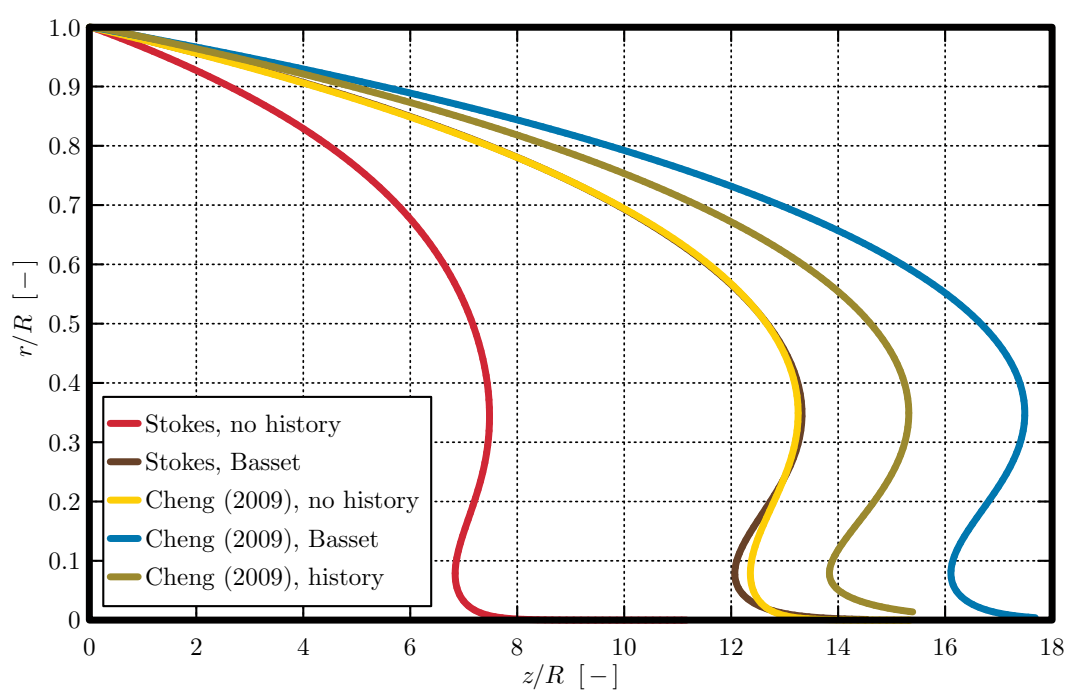

Figure 6.2: Radial position of oil-droplet as a function of axial position during motion in swirling flow field of figure 6.1.

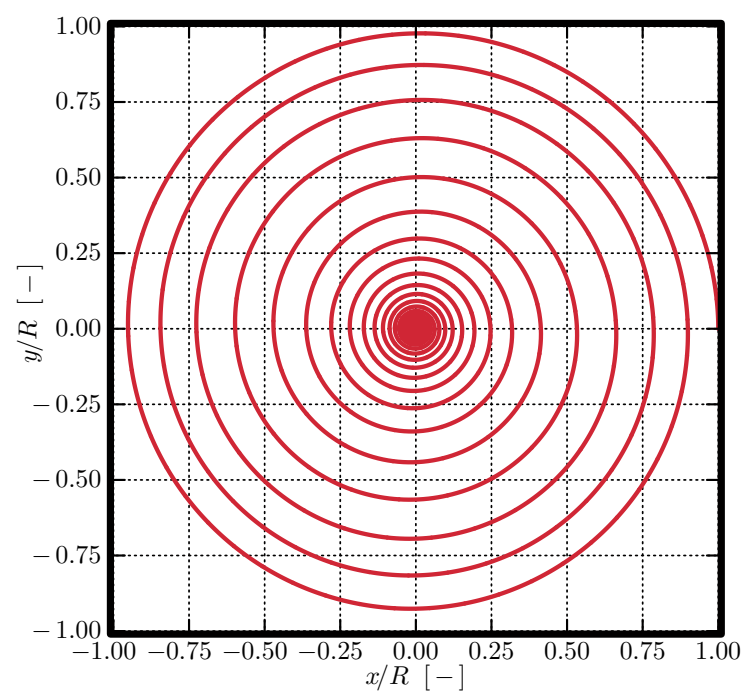

Figure 6.3: Oil-droplet trajectory projected on the $x, y$-plane for the oil-droplet released at $\left(\frac{x}{R}, \frac{y}{R}\right)=(1,0)$. Stokes, no history force. 


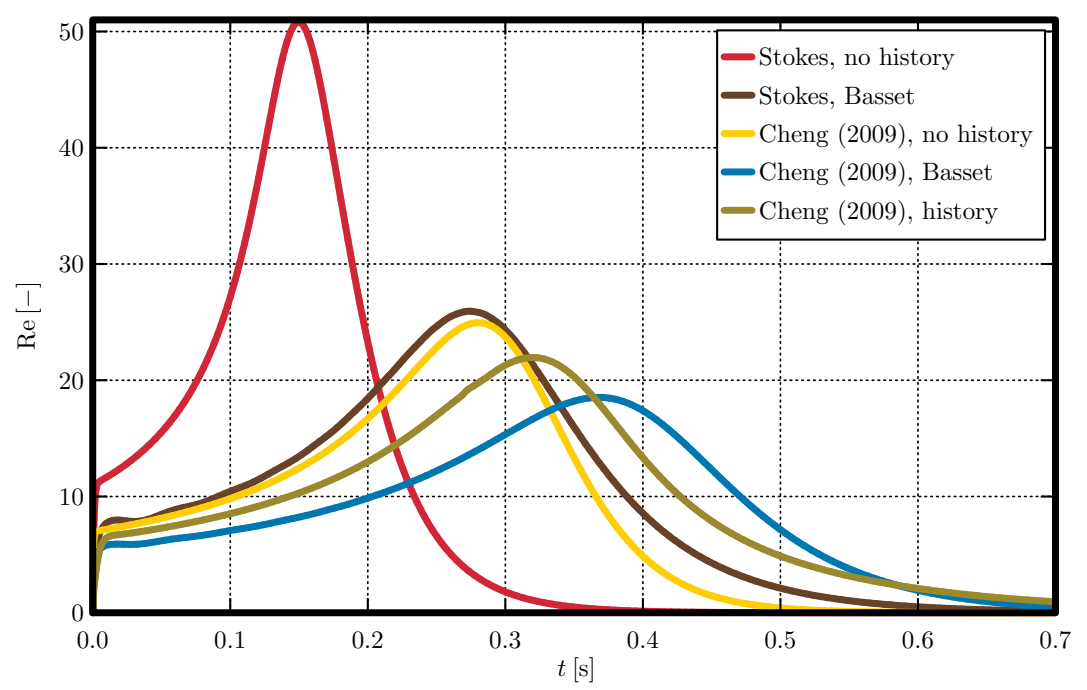

Figure 6.4: Reynold number as function of time encountered by droplet during motion in the swirling flow field.

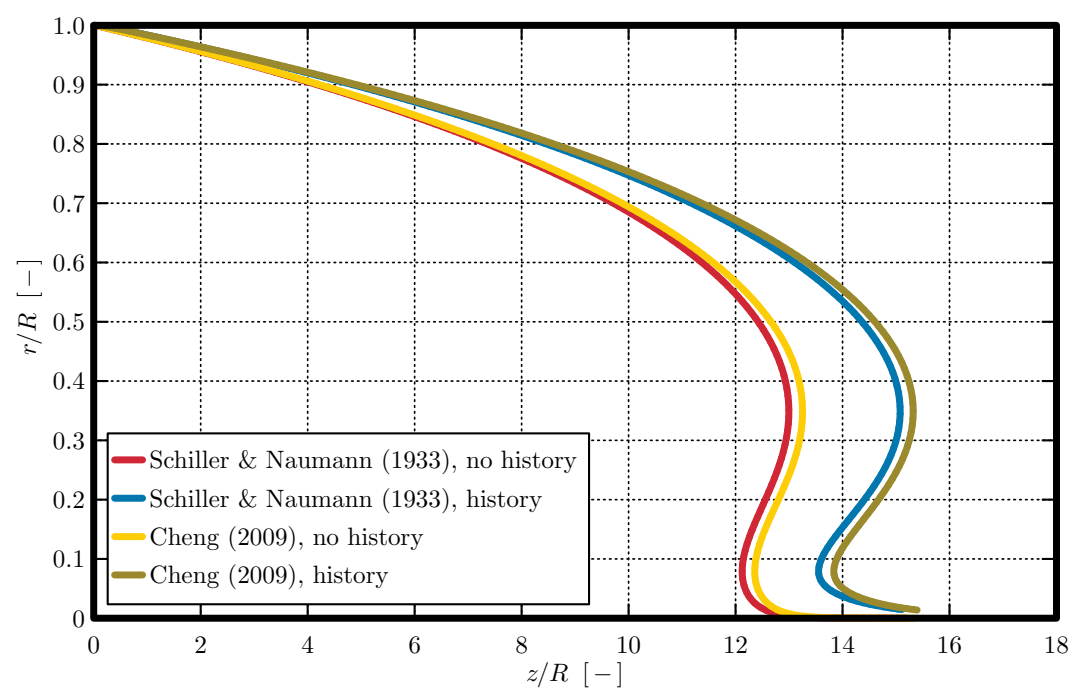

Figure 6.5: Comparison of radial position predicted using drag factors proposed by Cheng (2009) [10] and Schiller \& Naumann (1993) [35]. 
device not taking into account the non-Stokesian effects, would have been a factor 1.8 too short for the considered oil-droplet to be captured in the central region. This observation is supported by the Reynolds number encountered by the droplet along the particle trajectory. Figure 6.4 shows that Reynolds numbers for the trajectory computed including non-Stokesian effects go up to 25, while the Reynolds number for the simulation only taking into account Stokes drag reaches a Reynolds number of approximately 50. These Reynolds numbers are clearly not in the range for which Stokes drag is valid, resulting in the observed behaviour of the particle trajectories.

It is also observed that, for the current application, the history force has a significant influence on the particle trajectory. Similar to the simulations for the settling sphere, the simulations for oil-droplets in swirling flow show that the Basset history force overestimates the influence of the history force. However, the proposed Reynolds dependent history kernel still increases the length of the device, required for separation, with a factor 1.15. Moreover, it can be observed that the particle trajectory following from the simulation using only Stokes drag but including the Basset history force is remarkably close to the particle trajectory for the simulation without accounting for the history force, but with non-Stokesian effects for the drag. This indicates that a drag-like algebraic expression might be used to approximate the effects of the history force on the particle trajectory.

Many commercial applications use the adapted form of the drag factor initially proposed by Schiller \& Naumann (1993) [35]. This drag factor, equation (3.64), clearly deviates from the drag factor used in the particle simulations presented in figure 6.2. Moreover, it should be noted that the Reynolds numbers are still in the region in which the original expression proposed by Schiller \& Naumann is still valid. Therefore, simulations have been performed using this drag factor, in order to assess the influence of a different drag factor. The results presented in figure 6.5 show visible differences between predictions using different drag factors. However, the influence is minor compared to the influence of the history force. Therefore, if the history force would be neglected for a swirling flow, the choice of the drag factor is not that important as long as it takes into account non-Stokesian effects sufficiently. The accuracy of the expression proposed by Cheng (2009) is reported to be higher than that of Schiller \& Naumann (1993) [10]. Moreover, it does not have a singularity in its first derivative. Therefore we conclude that this expression should be favoured over the more traditional expression used in many commercial applications.

Simulations performed including the Saffman lift force showed no visual differences in particle trajectories. Therefore, lift forces are deemed insignificant 
for the current application in which the angle between the relative motion of the particle with respect to the vorticity of the flow field is such that lift forces remain small.

\subsubsection{An efficient approximation of the history force}

An approximate expression for the history force, taking only the instantaneous information of the particle motion and velocity field into account, will result in a huge reduction of computational work-load. However, this can only be done in specific cases if the motion of the particle is known approximately. In the preceding section it has been observed that the influence of the Basset history force could be approximated by the inclusion of the Reynold dependent terms of the drag force for that specific case. This indicates that a similar approach might be used to correct the instantaneous drag for history effects. To investigate the possibility of such a computationally less expensive alternative in the case of swirling flow, the history force will be investigated for the motion of an oil droplet in swirling flow, predicted using the correlation by Cheng (2009), considered in the preceding section.

In figure 6.6 the magnitude of the history force is presented as a fraction of the magnitude of the sum of the magnitudes of the history and the drag force as a function in time, i.e.

$$
\boldsymbol{F}_{\text {H,relative }} \equiv \frac{\boldsymbol{F}_{\mathrm{H}}}{\left\|\boldsymbol{F}_{\mathrm{H}}\right\|+\left\|\boldsymbol{F}_{\mathrm{D}}\right\|} .
$$

Oscillating behaviour occurs at about $t \approx 0.28[s]$. This behaviour is also observed in the velocity, presented in figure 6.4 , where a slight, almost invisible, bumb in the velocity profile is observed at that same time. The cause of this behaviour is still under investigation. However, it is probably related to the change in acceleration in both radial and axial direction. The change occurs in the region where the axial velocity of the flow field has significantly decreased with respect to its initial value when the particle was near the wall of pipe. Moreover, the derivative of the azimuthal velocity with respect to the radius is decreasing in that the driving pressure gradient force is starting to change in behaviour.

Following an initial peak in the relative history force, the relative history force remains nearly constant. This suggests that in that region the history force could be approximated by a scaled drag force. After entering the region of solid body rotation, the particle is decelerating and the drag force reduces. As the relative history force increases, this indicates that the history force does 


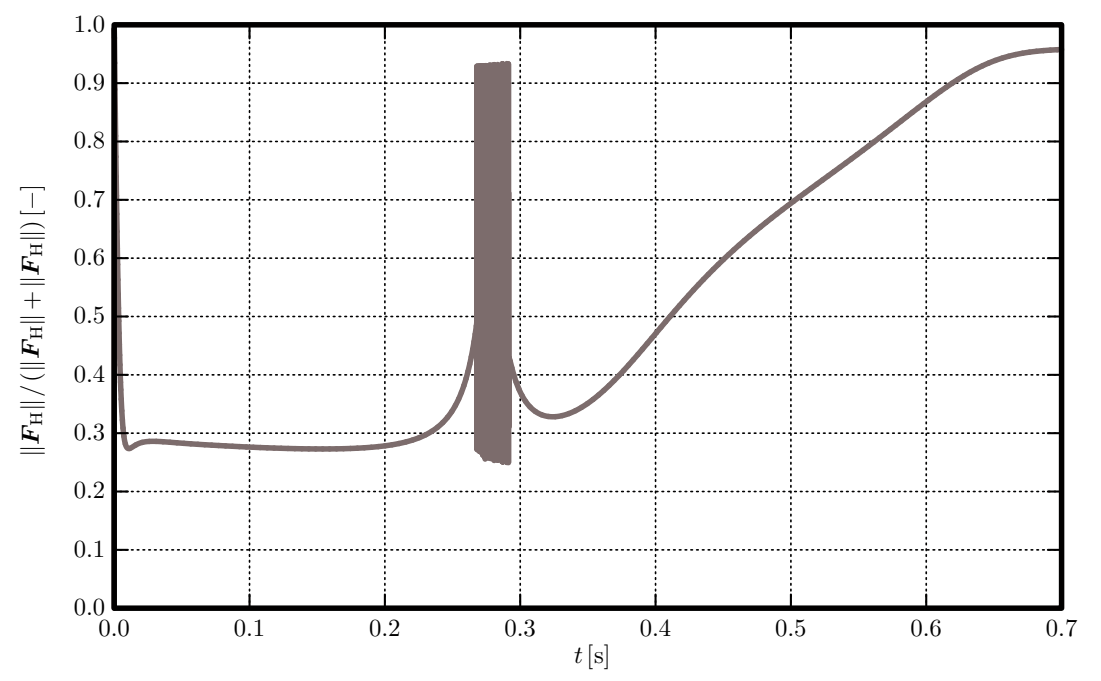

Figure 6.6: Magnitude of the history force relative to magnitude of the sum of the magnitudes of the history and the drag force as a function of time.

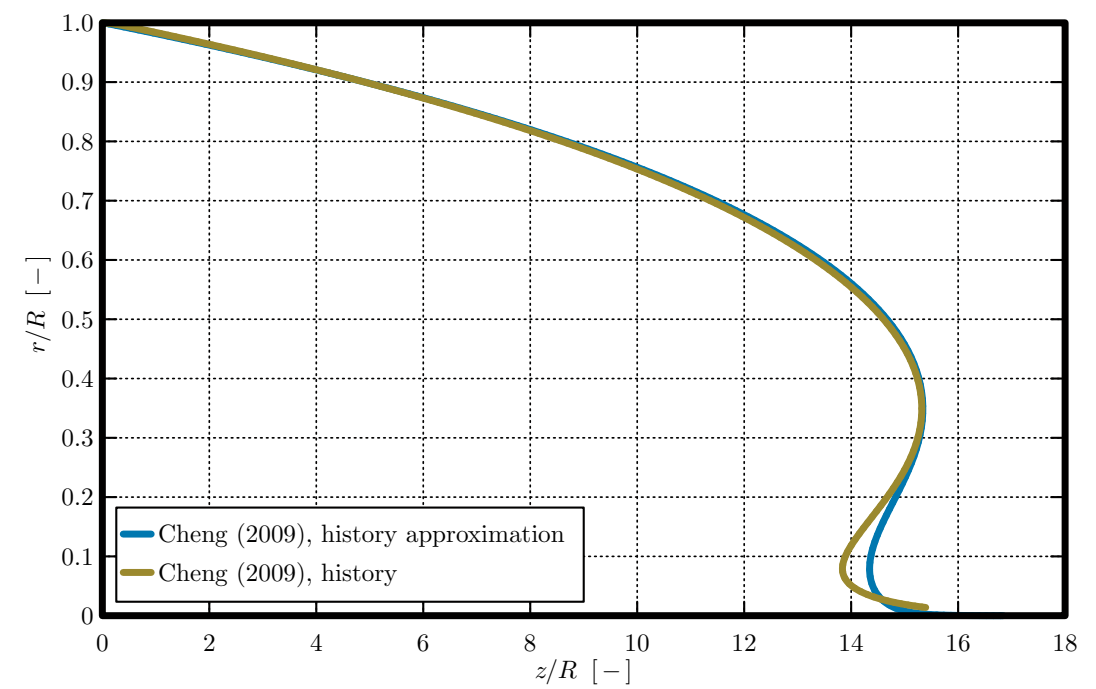

Figure 6.7: Result for the drag like approximation of the history force, equation (6.5), compared to the result with the actual history force. 
not reduce at the same rate as the drag force. For this region, a drag force like expression cannot be used to approximate the history force. However, the influence of both forces in the latter region is minor compared to the influence in the first part of particle motion. Approximation of the history force by a drag force results in an equation for a factor $\alpha_{\mathrm{H}}$ as

$$
\left\|\boldsymbol{F}_{\text {D,relative }}\right\| \approx 0.28 \equiv \frac{\alpha_{\mathrm{H}}\left\|\boldsymbol{F}_{\mathrm{D}}\right\|}{\left(1+\alpha_{\mathrm{H}}\right)\left\|\boldsymbol{F}_{\mathrm{D}}\right\|},
$$

which results in $\alpha_{\mathrm{H}} \approx 0.39$. However, using a value of $\alpha_{\mathrm{H}}=0.218$ turns out to give the best results for this specific case. Figure 6.7 shows resulting particle trajectories in the $r, z$-plane one when using the history force in equation (3.72) with the kernel from equation (3.78) and one when using the approximation for the history force

$$
\boldsymbol{F}_{\mathrm{H}} \approx 0.218 \boldsymbol{F}_{\mathrm{D}}
$$

As can be expected from the behaviour of the relative history force, the first part of the trajectory can be predicted accurately using the approximated history force, while for the last part, the influence of the history force is underestimated. However, the last part of the particle trajectory is of less interest than the first part of the trajectory, as the first part is the major factor in determining separator dimensions.

The discrepancy between the expected result for the factor in the approximation needs further investigation. The factor for the approximation of the history force is probably dominated by the importance of the acceleration effects in both axial and azimuthal direction, resulting in a deviation from the expected value if one of those directions dominates the result for the $z, r$-profile.

The simulations in present research have been performed for a single droplet size, release at a specific location in one specific swirling flow field. The factor multiplying the drag is expected to depend on droplet size and magnitude of the swirl. Furthermore, the result in the region with solid body rotation might be improved by using a factor that linearly increases with decreasing radius, e.g.

$$
\alpha_{\mathrm{H}}=\beta_{\mathrm{H}}+\gamma_{\mathrm{H}} \max \left(\frac{r_{\text {Solid body }}-r}{R}, 0\right),
$$

where $r_{\text {Solid body }}$ is the radius at which the flow changes in behaviour from solid body rotation to that of a potential flow vortex.

In this research, the history force has been related to the drag force during particle motion in the swirling flow field. For other flow fields an approach based 
on the instantaneous velocity might not be well suited. However, the approach may be extended by having the history force depending only on the value of the instantaneous acceleration, or a multiplication with a function depending on the relative acceleration and a function depending on the instantaneous velocity. In the latter method of approximating the history force, the force reduces to zero when the acceleration reduces to zero, as it should. Therefore a combination of a drag-like and added mass-like expression is a potential candidate to increase the accuracy of the approximation of the history force. Moreover, a reduced history force integral, using a history time cut-off region as proposed by Loth and Dorgan [16, 28], might result in sufficient reduction of computation time for an adequate approximation of the history force. Furthermore, an efficient coarsening scheme for the long-time decay region could also be considered for decreasing computational effort of computing the integral in the history force, while retaining both accuracy and smoothness of the solution. 



\section{Chapter 7}

\section{EFFICIENT COLLISION DETECTION}

For the application of swirling flow to the treatment of produced water presented in this thesis, it has been assumed that the mixture can be considered a dilute multiphase fluid in most parts of the domain. For such fluids it is appropriate to apply one-way coupled Lagrangian particle tracking for the prediction of oil-droplet trajectories. However, if the concentration of droplets becomes large, four-way coupling needs to be taken into account. Due to the related increases in computational cost, Lagrangian particle tracking may become infeasible. Moreover, Lagrangian particle tracking with only one-way coupling may not predict separation efficiency correctly due to particle-particle interaction. One-way coupling does not take into account coalescence, which increases separation efficiency. Furthermore, one-way coupling does not take into account the effect of hindering, in which droplets obstruct other droplets reducing separation efficiency. Also the influence of the droplets on the macroscopic flow field becomes important for higher particle concentrations. To assess the coalescence probability and the validity of the assumption of a dilute multiphase fluid, as well as to prepare for four-way coupling, a collision detection mechanism is required.

The computational cost of collision detection in Lagrangian particle tracking will become infeasible when a large number of particles is employed. We show in this study that the workload of a straightforward collision detection scheme scales with $\mathcal{O}\left(N_{\mathrm{p}}^{2}\right)$, with $N_{\mathrm{p}}$ the number of particles. However, the importance 
of particle-particle interactions becomes dominant for a relatively large number of particles, for which such a scheme is very expensive. For that reason, a computationally more efficient scheme for collision detection is desirable.

This chapter describes the implementation and resulting properties of such an efficient scheme for detection of collisions between particles that are forced by volumetric forces and by a flow field. The purpose of the presented collision detection scheme is to substantially reduce the computational workload as compared to the straightforward collision scheme such that it has an expected work-load of $\mathcal{O}\left(N_{\mathrm{p}} \ln N_{\mathrm{p}}\right)$. The collision detection algorithm is first validated against the case of an ensemble of spheres that would collide with each other while settling in water due to gravity. Subsequently, the scheme is applied to the motion of droplets in a swirling flow field typical for produced water treatment. Possible improvements of the scheme and the relevance of the method for separator design will be discussed. The collision detection scheme, as well as its results have been presented in van Eijkeren et al. (2014) [21].

\subsection{Collision detection scheme}

A collision between two spherical particles takes place if the distance between the centres of these particles is less than the sum of their radii. Similarly, a significant effect of one particle on the other particle is observed if the distance between the centres of influence is less than the sum of its radii, i.e. the radii of the spheres of influence. Therefore, the condition for such an event becomes

$$
\left\|\boldsymbol{x}_{1}-\boldsymbol{x}_{2}\right\| \leq \frac{D_{1}}{2}+\frac{D_{2}}{2}
$$

where $\boldsymbol{x}_{i}$ is the $i$-th particle position and $D_{i}$ is the diameter of the $i$-th particle, or the diameter of the associated sphere of influence, in case of collisions or significant influence, respectively.

\subsubsection{Straightforward implementations}

A straightforward method to implement collision detection is

1. Prediction of new particle positions;

2. For each particle $i$, check for all other particles $j$ the condition; $\left\|\boldsymbol{x}_{i}-\boldsymbol{x}_{j}\right\| \leq \frac{1}{2}\left(D_{i}+D_{j}\right)$; 
3. If the condition is true the number of collisions $N_{\mathrm{c}}$ is increased by $\frac{1}{2}$;

Note that the number of collisions is increased by $\frac{1}{2}$ as both the collision of particle $i$ with particle $j$ as well as the collision of particle $j$ with particle $i$ are counted separately. The work-load $W_{\mathrm{BF}}$ of this so-called brute force scheme is

$$
W_{\mathrm{BF}}=N_{\mathrm{p}}\left(N_{\mathrm{p}}-1\right) W_{\text {Condition }}
$$

where $W_{\text {Condition }}$ is the work load of calculating the distance and checking the condition and where $N_{\mathrm{p}}$ is the number of particles. However, the scheme can be easily parallelized for speed-up resulting in a workload per processor core of order $\frac{N_{\mathrm{p}}}{p}\left(N_{\mathrm{p}}-1\right) W_{\text {Condition, }}$, with $p$ the number of processors.

The work load of the scheme can be reduced by a factor $\frac{1}{2}$ using a slightly modified implementation

1. Start with particle $i=0$;

2. Prediction of new particle position for particle $i$;

3. For each particle $j<i$ i.e. the particles that have been advanced, check the condition;

$$
\left\|\boldsymbol{x}_{i}-\boldsymbol{x}_{j}\right\| \leq \frac{1}{2}\left(D_{i}+D_{j}\right)
$$

4. If the condition is true the number of collisions $N_{\mathrm{c}}$ is increased by 1 ;

The scheme can also be employed after all new particle positions have been calculated. Depending on the way the scheme is parallelized one or the other might be more efficient. The work load $W_{\mathrm{S}-\mathrm{BF}}$ of this so-called semi brute force scheme is

$$
W_{\mathrm{S}-\mathrm{BF}}=\frac{N_{\mathrm{p}}}{2}\left(N_{\mathrm{p}}-1\right) W_{\text {Condition }},
$$

\subsubsection{Proposed implementation}

It can be observed that the work-load of both schemes is $\mathcal{O}\left(N_{\mathrm{p}}^{2}\right)$. Collision detection is important for large $N_{\mathrm{p}}$, for which straightforward implementation is computationally very expensive. Therefore, a scheme is proposed that reduces the work-load to approximately $\mathcal{O}\left(N_{\mathrm{p}} \ln N_{\mathrm{p}}\right)$. The scheme first assigns particles to so-called containers, and only checks for collisions inside these containers. The scheme, presented in figure 7.1, is

1. Start with particle $i=0$; 
2. Prediction of new particle position for particle $i$;

3. Determine the regions the particle is potentially in, depending on a cubed bounding box around the particle;

4. For each such region

- If a container containing particles in such a region does not exist, create a container and add the particle;

- If a container containing particles in such a region does exist, check whether a collision has occurred, if so increase the number of collisions $N_{\mathrm{c}}$ by one.

Keep track of collided particles during this step to avoid counting collisions by the same particles detected in more than one container.

Assuming an $\mathcal{O}\left(N_{\mathrm{p}} \ln N_{\mathrm{p}}\right)$ algorithm to assign the particles to regions, and a grid that is properly sized, the workload $W_{\mathrm{BS}}$ of this so-called box-scheme will be

$$
W_{\mathrm{BS}} \sim\left(N_{\mathrm{p}} \ln N_{\mathrm{p}}\right) W_{\text {Condition }} .
$$

The scheme is not only more efficient, it also has the potential to be used during a multi-grid summation for long distance particle-particle interactions, e.g. many-body potential summations [25], for which the influence of particles is first redistributed to the nodes of a Cartesian grid. On the finest level, the Cartesian grid is related to a cut-off distance for the used interaction function. The sphere defined by this cut-off distance can be seen as the sphere of close-distance influence, in which long-distance approximations by a polynomial function starts to deviate significantly from the actual interaction function. In the final step of such a multi-grid summation approach a correction needs to be applied inside this sphere of influence to account for that deviation from polynomial behaviour.

\subsection{Collision detection for settling spheres}

In order to assess the efficiency of the proposed collision detection scheme, first collision detection has been performed on iron spheres of random size settling in water. The spheres of different sizes will have different terminal velocities. Therefore, collisions will occur when particles overtake each other and they overlap when seen in the plane perpendicular to the direction of settling motion. Collisions will be counted, however, the spheres will not interact with each other 
(1)
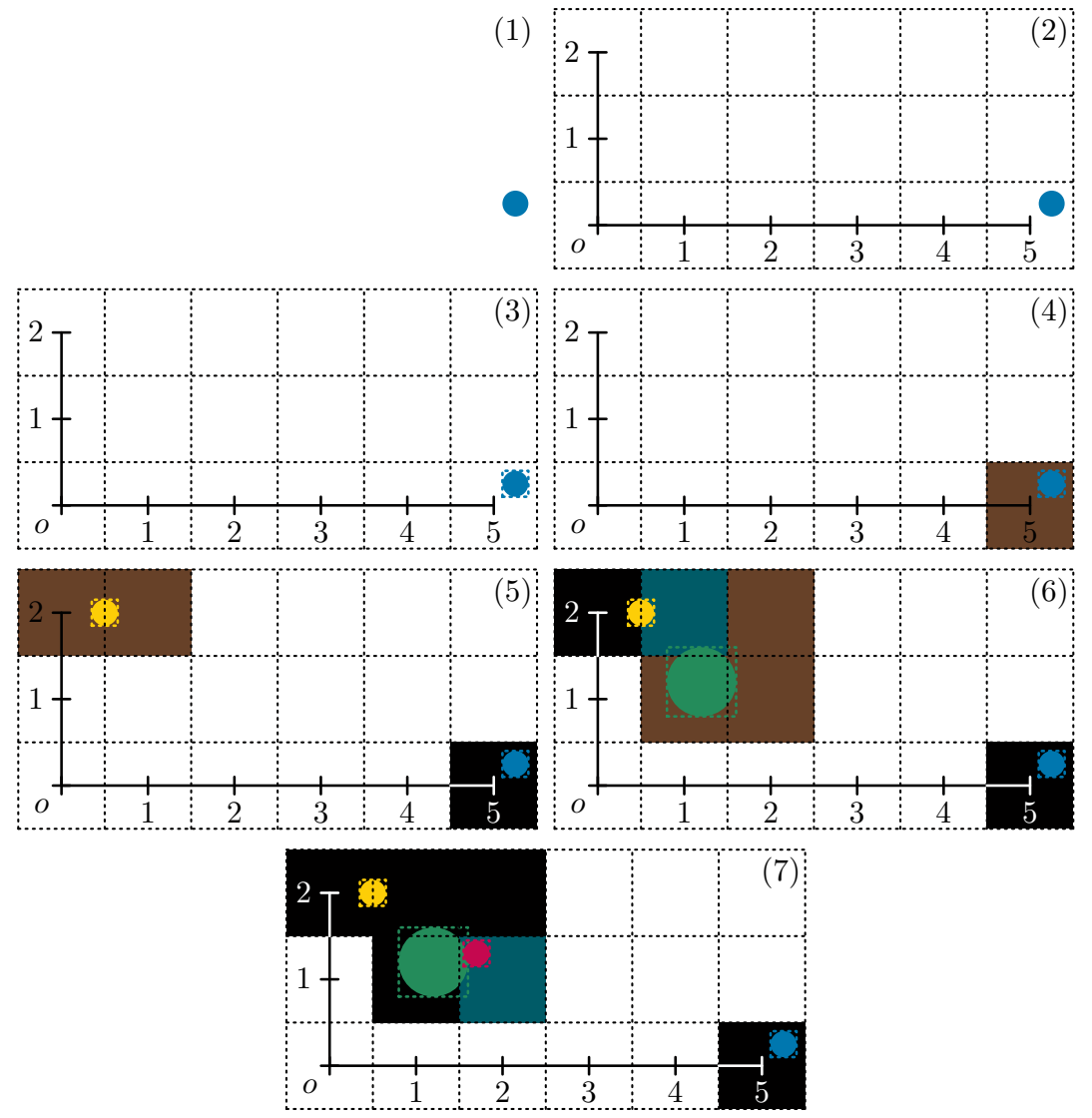

Figure 7.1: Proposed collision detection scheme for a small domain in 2D:

1. A new particle position is calculated.

2. A virtual grid divides the space in regions.

3. A bounding box around the particle determines the potential regions.

4. A container is created for the region the particle is in.

5. The process is repeated for a next particle. Containers are created for new regions.

6. For the existing region the particle is added to the container. A distance check to all other particles in the container is performed.

7. First time a collision is detected. 


$$
\begin{array}{cccc}
\rho_{\mathrm{H}_{2} \mathrm{O}}\left[\mathrm{kg} \mathrm{m}^{-3}\right] & \nu\left[\mathrm{m}^{2} \mathrm{~s}^{-1}\right] & D_{\max }[\mathrm{m}] & \rho_{\text {iron }}\left[\mathrm{kg} \mathrm{m}^{-3}\right] \\
997 & 8.96 \times 10^{-7} & 1 \times 10^{-3} & 7850
\end{array}
$$

Table 7.1: Material attributes of the continuous phase and the settling spheres.

in presented simulations and will continue as if the other particle is not present. Moreover, as long as the particles remain overlapping, no new collision will be counted for these specific combination of particles. Due to the difference in terminal velocity, the non-interacting spheres will gradually separate in size depending on their terminal velocity. Note that coalescence, collision modeling and long distance particle interaction are not part of this research.

\subsubsection{Material properties and geometry}

The material properties of the iron spheres and water as used in the simulation are presented in table 7.1. The gravitational acceleration has been taken as $g=9.81\left[\mathrm{~m} \mathrm{~s}^{-2}\right]$ and acts in the direction of the $z$-axis. The diameters of the iron spheres have been randomly distributed using a uniform distribution function with $0[\mathrm{~m}]<D \leq D_{\max }$. With such a uniform distribution function all diameters in that range are expected to occur as often as all other diameters in that range for large enough ensembles. The particles have been initialized at $t_{0}=0[\mathrm{~s}]$ with a uniformly distributed random height $0[\mathrm{~m}] \leq z_{i, 0} \leq 1000 D_{\max }$. Four cases have been considered for the distribution of the spheres in the projected plane perpendicular to the $z$-axis:

1. Spheres are located on the $z$-axis with $x_{i}=0[\mathrm{~m}]$ and $y_{i}=0[\mathrm{~m}]$

2. Uniform random distribution in both $x_{i}$ and $y_{i}$ with

$$
\begin{aligned}
& \text { (a) }-D_{\max } \leq x_{i}, y_{i} \leq D_{\max } \\
& \text { (b) }-2 D_{\max } \leq x_{i}, y_{i} \leq 2 D_{\max } \\
& \text { (c) }-4 D_{\max } \leq x_{i}, y_{i} \leq 4 D_{\max }
\end{aligned}
$$

Note that the position in the $x, y$-plane does not change, as the motion of the particles, due to gravity, is only in the $z$-direction. 

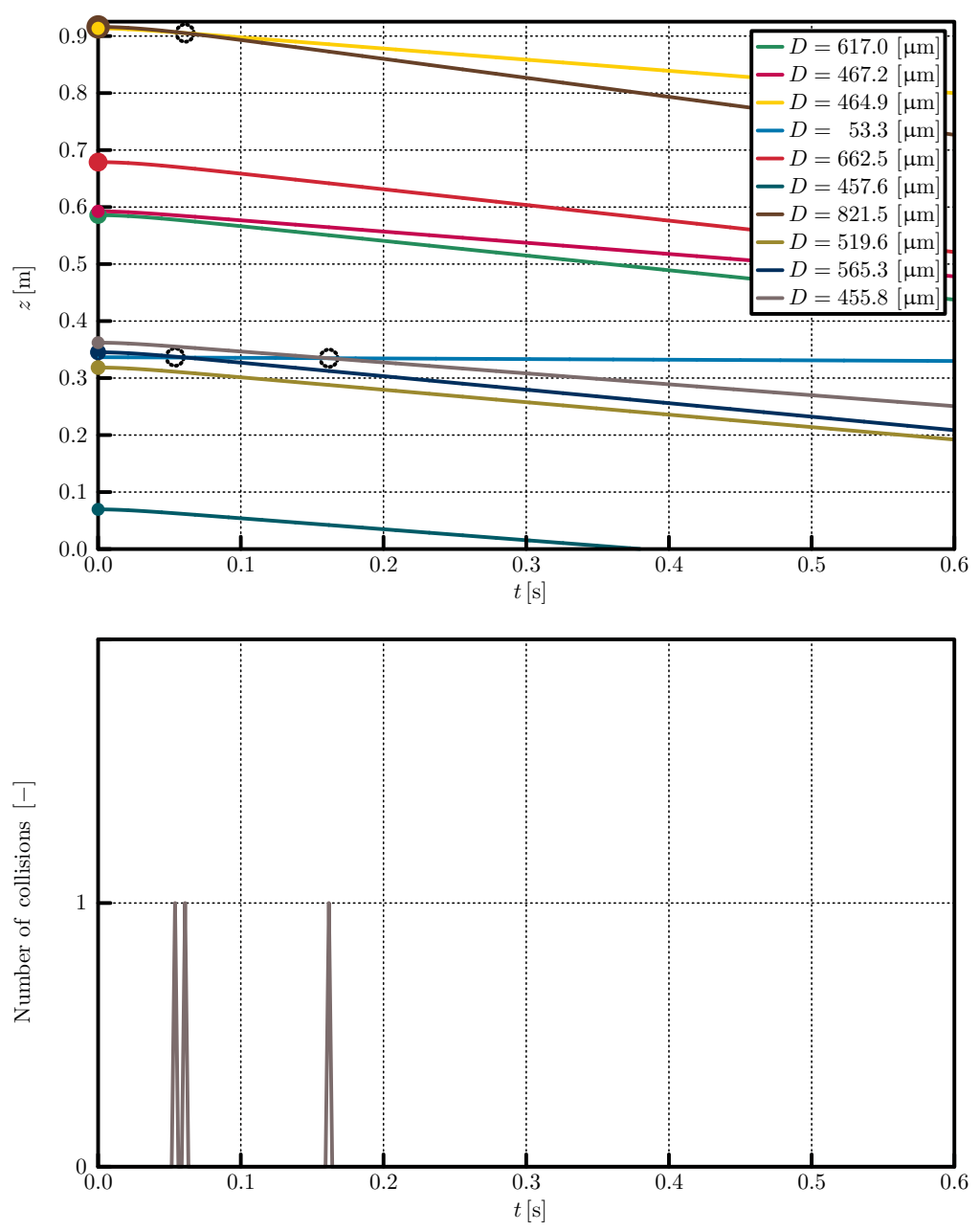

Figure 7.2: Particle trajectories for the case of 10 settling spheres of random diameter with an initial random height exactly aligned with the $z$-axis. The upper graph shows the vertical position of the particles as a function of time. The lower graph shows the number of collisions as a function of time. The moments of crossing particle trajectories are indicated. Furthermore, the scaled size of the particles is shown at the time of release. 


\subsubsection{Results and discussion}

\section{Validation}

To validate the collision detection scheme, the set-up of case 1 is considered with 10 settling spheres. A specific seed for the random number generator is selected in order to have some collisions in a limited time-domain. Results for this simulation are presented in figure 7.2. The upper graph shows the particle trajectories, i.e. $z$-location of the spheres as a function of time. In the upper graph the relative size of the spheres with respect to each other and the time when two trajectories cross are also indicated. The lower graph shows the number of collisions counted each time-step. From these graphs it is observed that each time a trajectory crosses another trajectory, the scheme correctly detects one collision. Therefore, it can be concluded that the scheme for this case is working correctly.

\section{Performance}

The working scheme is now investigated for its performance. The mesh size $h$ of the regions in which the domain is subdivided will be an important parameter for the efficiency of the scheme. Choosing the mesh size too small will lead to many cells per particle, which increases the work-load. However, choosing the mesh size too large will result in many particles per sub-region, ultimately leading to a performance close to that of the straightforward scheme. Therefore, the influence of this mesh size has been investigated.

For all cases, cases $1,2.2 \mathrm{a}, 2.2 \mathrm{~b}$ and $2.2 \mathrm{c}$, simulations have been performed with 1024 particles. The second collision detection method with workload $W_{\mathrm{S}-\mathrm{BF}}$ has been used as a reference. To ensure similar behaviour in all simulations, the random values have been generated with a specific seed, resulting in the same random distribution for all simulations of a specific case.

Calculations using the reference method take in the order of 620 [s] on a desktop using a single core. Figure 7.3 shows the results of the simulations. For all cases the speed-up gained by using the proposed scheme shows similar behaviour. For a small mesh sizes, the size becomes too small, reducing performance. When the particles span a larger area in the plane perpendicular to the direction of motion, the reduction in performance due to this effect is less dominant. Many particles are much smaller than $D_{\max }$ and will not cover too many cells. Moreover, in the projected plane perpendicular to the $z$-axis, the reduced mesh size helps preventing unnecessary collision checks when particles will overtake each other without colliding. When the mesh size becomes too large, the 


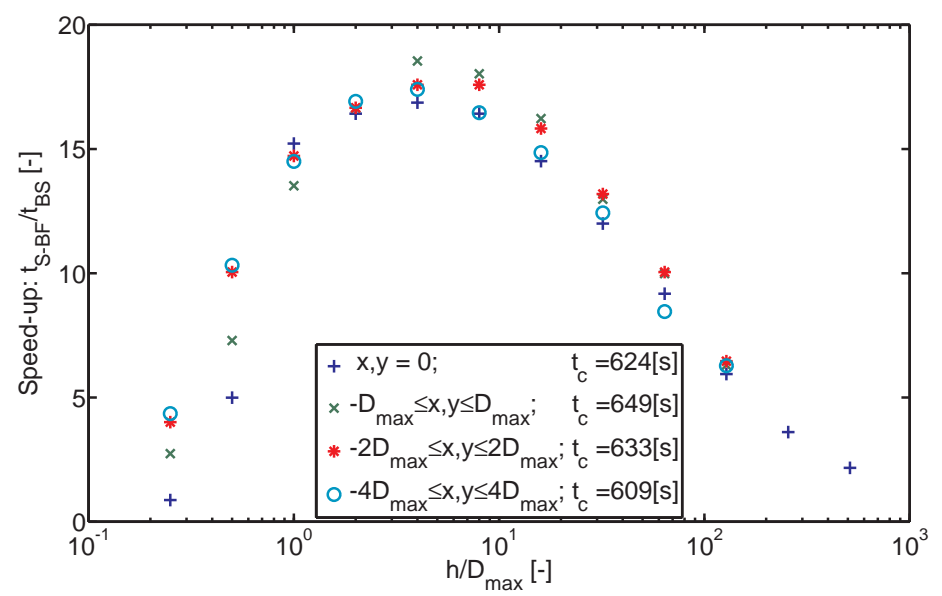

Figure 7.3: The speed-up for 1024 particles gained by using the proposed scheme with workload $W_{\mathrm{BS}}$ compared to the straightforward scheme with workload $W_{\mathrm{S}-\mathrm{BF}}$ as a function of the size $h$ of the regions used in the proposed scheme. The reference time $t_{\mathrm{c}}=t_{\mathrm{S}-\mathrm{BF}}$ is also shown for each case.

performance decreases until all particles are contained in one region, reducing the proposed method to the reference method with some extra overhead.

The maximum speed-up, when the scheme performs approximately 16 to 17 times faster can be observed at a mesh size of $h=4 D_{\max }$, where $t_{\mathrm{S}-\mathrm{BF}}=624[\mathrm{~s}]$ versus $t_{\mathrm{BS}}=37[\mathrm{~s}]$. For all cases the optimal mesh size is approximately the same, although for some cases the optimum appears to be at slightly higher mesh size. Note that, for different particle distributions, the method will perform differently. However, the scheme performs well for a fairly large range of meshsizes. This will make it possible to have a good performance even for a nonoptimal choice of the mesh-size.

Using the obtained optimal $h=4 D_{\max }$, the behaviour of the two schemes is investigated as a function of the number of particles. The resulting calculation time for the scheme with workload $W_{\mathrm{S}-\mathrm{BF}}$ as well as the calculation time for the proposed scheme are presented in figure 7.4. Moreover, a trend line is presented for both schemes based on the workload in equations (7.3) and (7.4). For higher number of particles, the predicted workload agrees well with the resulting calculation times. For low particle numbers, calculation time is not 


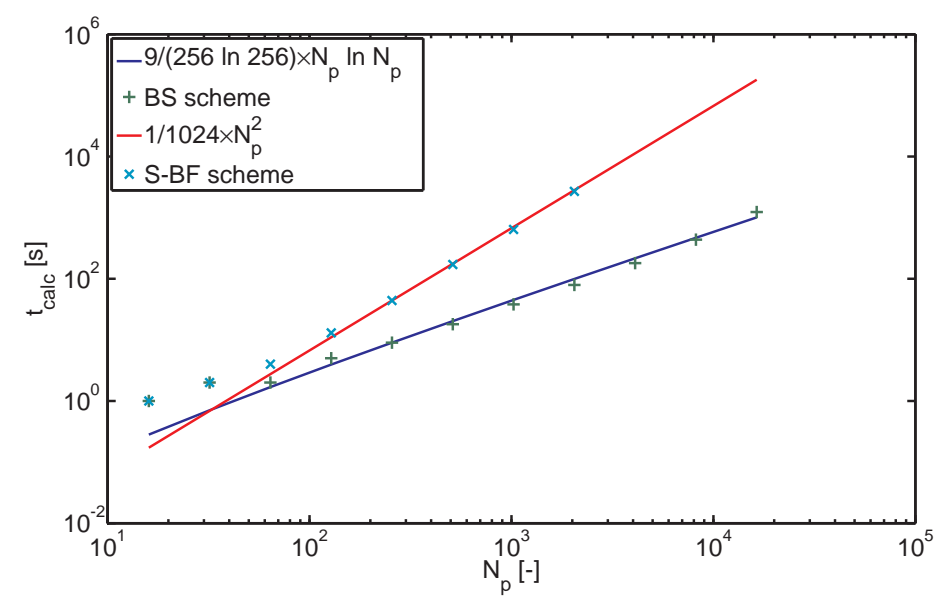

Figure 7.4: The calculation time as a function of the number of spheres using $\frac{h}{D_{\max }}$. A trend line is presented for each scheme based on its theoretical workload.

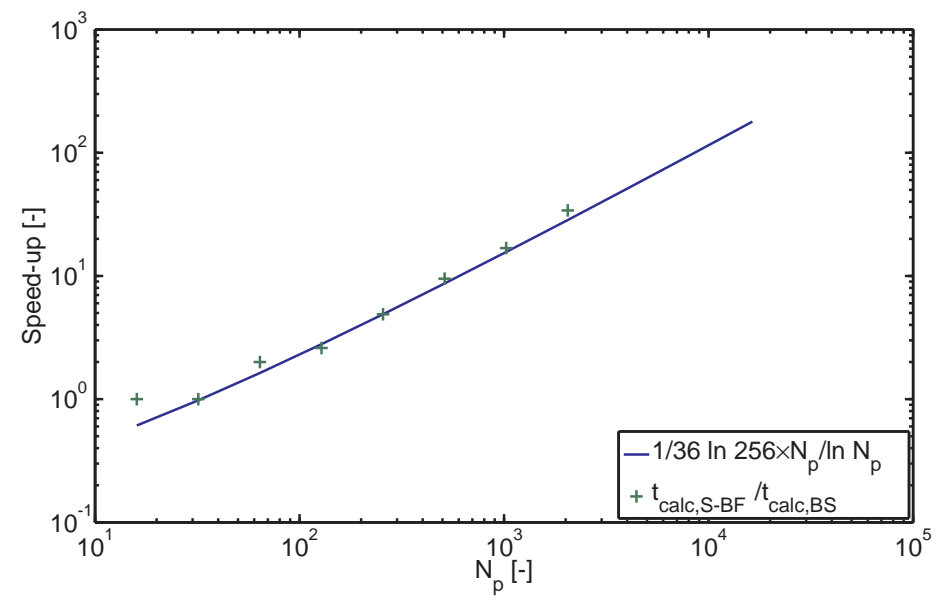

Figure 7.5: The speed-up obtained with the proposed scheme as a function of the number of spheres. A trend line is shown, which is the ratio of the trend lines presented in figure 7.4 . 


$\begin{array}{cccc}\tau_{\nu}[\mathrm{s}] & \Gamma\left[\mathrm{m}^{2} \mathrm{~s}^{-1}\right] & \nu\left[\mathrm{m}^{2} \mathrm{~s}^{-1}\right] & R[\mathrm{~m}] \\ 500 & 2 & 8.92 \times 10^{-7} & 0.05 \\ \tau_{\nu, 1}[\mathrm{~s}] & U_{\infty}\left[\mathrm{m} \mathrm{s}^{-1}\right] & U_{0}\left[\mathrm{~ms}^{-1}\right] & U_{1}\left[\mathrm{~m} \mathrm{~s}^{-1}\right] \\ \frac{250}{3} & 2 & 6 & \frac{7}{2}\end{array}$

Table 7.2: Parameters for the swirling flow field, used to mimic a flow field typical for produced water treatment using a swirling flow separator.

$$
\begin{array}{cccc}
\rho_{\mathrm{H}_{2} \mathrm{O}}\left[\mathrm{kg} \mathrm{m}^{-3}\right] & \nu\left[\mathrm{m}^{2} \mathrm{~s}^{-1}\right] & D_{\max }[\mathrm{m}] & \rho_{\text {oil }}\left[\mathrm{kg} \mathrm{m}^{-3}\right] \\
997 & 8.92 \times 10^{-7} & 2 \times 10^{-4} & 800
\end{array}
$$

Table 7.3: Material properties of the continuous phase and the oil droplets.

yet dominated by collision detection, resulting in deviation from the predicted workload behaviour. The speed-up gained by using the proposed scheme is presented in figure 7.5 together with the ratio of the trend lines for the workload in figure 7.4. It can be observed that the predicted speed-up of $\mathcal{O}\left(\frac{N_{p}}{\ln N_{p}}\right)$ is a good estimation of the actual speed-up of the method.

It can be concluded that the proposed scheme is working and has the predicted efficiency of $\mathcal{O}\left(N_{\mathrm{p}} \ln N_{\mathrm{p}}\right)$. Therefore, the scheme will now be employed for oil-droplets in a typical flow field observed during swirling flow oil-water separation.

\subsection{Collision detection in swirling flow}

For the case of collision detection, the flow field will be used presented in section 6.1, equation (6.1). The parameters for the swirling flow field are the same as the ones used in chapter 6 in table 6.1 and are repeated in this chapter in table 7.2 .

Numerical simulations have been performed to predict the particle trajectories of the oil-droplets in water. The properties of water and oil-droplets, as used in simulation of the oil-droplets in the swirling flow are shown in table 7.3. The material properties represent oil droplets in water at room temperature. For an initial investigation 1000 particles have been released with a random diameter $0<D \leq D_{\max }$. The particles are located at a random radius in- 

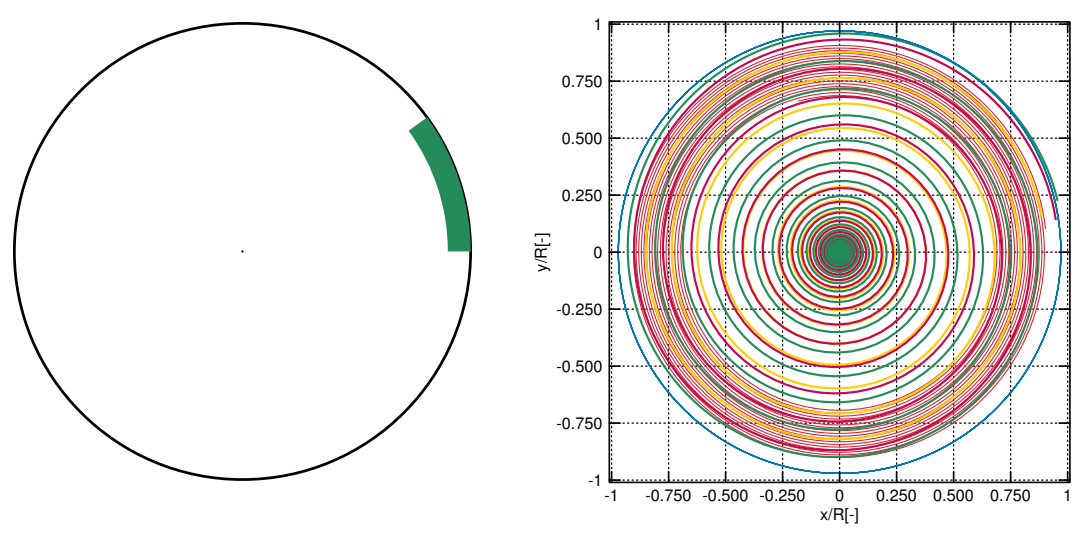

Figure 7.6: Initial position of the particles projected on the $x, y$-plane (left) and 5 particle trajectories projected on the $x, y$-plane obtained up to $t=0.6[\mathrm{~s}]$ (right)

side the range $0.9 R<r<R$ with a random azimuthal angle inside the range $0[\mathrm{rad}]<\theta<0.1 \pi[\mathrm{rad}]$ and a random axial distance from the inlet inside the range $0\left[[\mathrm{~m}]<z<R\right.$. A simulation has been performed up to $t_{\mathrm{end}}=0.6[\mathrm{~s}]$. Based on these conditions, the initial volume ratio of oil-water in the region of insertion is estimated to be $\frac{V_{\text {oil }}}{V_{\text {water }}} \approx 1 \times 10^{-3}$. The particle insertion position projected on the $x, y$-plane is shown in figure 7.6.

\subsubsection{Results and discussion}

Figure 7.6 shows the resulting particle trajectories for 5 selected particles up to $t=0.6[\mathrm{~s}]$. It is observed that some of the particles barely move at all in radial direction. These particles are very small oil-droplets with a relatively high drag coefficient with respect to the driving force in radial direction. Other particles reach the axis of rotation in only a few rotations. These are the large oil-droplets with a relatively low drag coefficient with respect to the driving force in radial direction.

In figure 7.7, the cumulative number of detected collisions is presented. It can be observed that in the initial $0.04[\mathrm{~s}]$ about 50 collisions take place. Sub- 


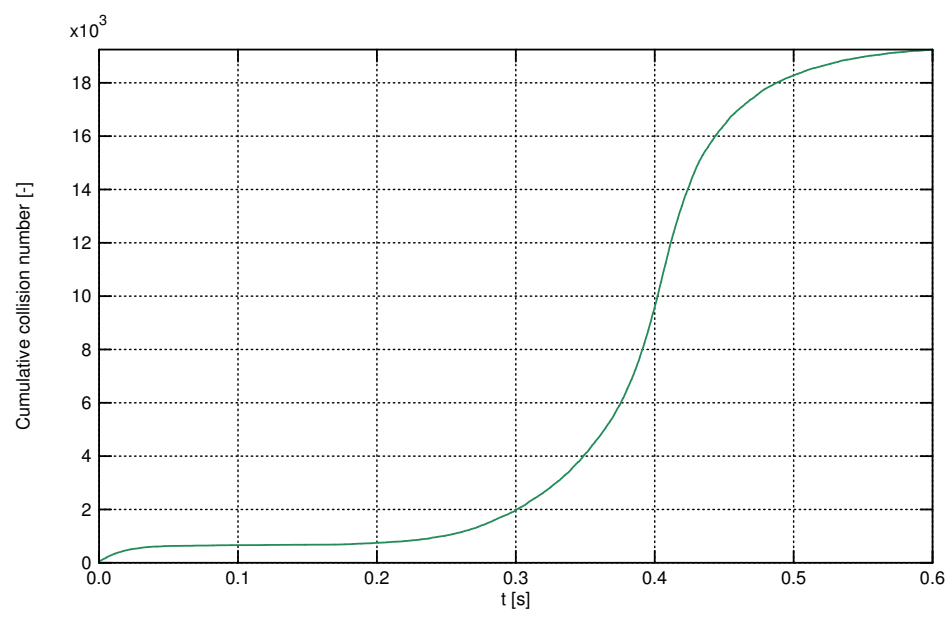

Figure 7.7: Cumulative number of detected collisions as a function of time for oil-droplets released from a small area in a swirling flow, presented in figure 7.6.

sequently the number of collisions very slowly increases until at about $0.2[\mathrm{~s}]$ where the number of collisions picks up until it very steeply increases up to about $0.5[\mathrm{~s}]$. Fewer and fewer new collisions are detected after $0.5[\mathrm{~s}]$. The total number of collisions detected is 19000 . However some particles might have been colliding more than once. Particles in a different flow regimes can be taken over again by particles that they have overlapped with before. Most likely near the axis of rotation, where particles might be colliding again with particles encountered before.

The simulations have been performed with the proposed scheme as well as with the scheme with workload $W_{\mathrm{S}-\mathrm{BF}}$. Due to a relatively large area in which particles are located, the proposed scheme performs much better with respect to the test case of the settling spheres. For 1000 particles the calculation with the proposed scheme was 100 times faster than the other scheme. This is much faster than was observed for the settling spheres. In the considered case, the particles are located in a relatively larger volume than for the case of the settling spheres. This results in much more particles being skipped for checking by the proposed scheme, resulting in a much higher speed-up. 



\section{CHAPter 8}

\section{LATTICE BOLTZMANN FOR WATER}

Fluid dynamics is the result of physical processes. In general the physics is described by equations that approximate the actual physical phenomena. For continuum fluid dynamics the Navier-Stokes equations are generally used to describe the macroscopic flow phenomena. In this chapter we apply the Boltzmann equation to the flow of water about a sphere.

Two approaches are presented to include non-ideal gas behaviour that is required to model the behaviour of water. Results of these approaches will be compared to results of an ideal-gas approach adapted to mimic the speed of sound of water. A novel approach is presented to include temperature dependent behaviour present in the Boltzmann equations. Further more this method facilitates decoupling of the mesh size from the time-step. Starting from the differential and integral equations for each approach, a Lattice Boltzmann discretization will be obtained as the result of the discretization of the integral and differential equations subject to the assumptions for various aspects of the equations.

\subsection{From molecular to macroscopic}

A fluid exists of molecules interacting with each other. Some of the molecules will also interact with molecules of the wall material. Moreover, the molecules are also subjected to forces like gravity. Each molecule has a specific velocity 
at a specific place and time. However, for a fluid consisting of a large number of molecules it becomes increasingly difficult to predict these specific velocities. The Navier-Stokes equations, that desctive conservation of mass, momentum and energy of the continuous average of the individual motion of the molecules, the so-called macroscopic quantities, are often used for practical flow problems. However, the Navier-Stokes equations neglect the discontinuous molecular nature of the fluid. With the increase in computational power, equations that are on a level between the continuous level and the molecular level, the so called mesoscopic level, become more and more feasible for application. Moreover, these equations make it possible to simulate phenomena not well represented by the Navier-Stokes equations, as well as phenomena where numerical simulations of the Navier-Stokes equations are not feasible due to complex boundary conditions.

The average quantities, used in the Navier-Stokes equations, can be seen as the average of a statistical distribution of molecules and molecular velocities. This implies that at a specific space and time it is most probable for a fluid to be in that specific state. The distribution function describing the probability to observe a molecule with velocity $\boldsymbol{c}$ when a molecule is observed at point $\boldsymbol{x}$ at time $t$ is $\mathcal{P}(\boldsymbol{c} ; \boldsymbol{x}, t)$ such that

$$
\int \mathcal{P}(\boldsymbol{c} ; \boldsymbol{x}, t) \mathrm{d} \boldsymbol{c} \equiv 1
$$

A specific number of molecules is located in an arbitrary but finite volume around position $\boldsymbol{x}$. Therefore, a number density $n(\boldsymbol{x}, t)$ exists for any arbitrary but finite volume located at $\boldsymbol{x}$ at time $t$. Scaling of the probability function with the number density leads to a probability density function $f(\boldsymbol{c} ; \boldsymbol{x}, t)$, defined as

$$
f(\boldsymbol{c} ; \boldsymbol{x}, t) \equiv n(\boldsymbol{x}, t) \mathcal{P}(\boldsymbol{c} ; \boldsymbol{x}, t)
$$

This distribution function is also used by, amongst others, Chapman and Cowling to formulate the kinetic theory of gases [8].

Macroscopic quantities considered in this research are the number density $n$ of molecules with mass $m$ resulting in the mass density $\rho$, the velocity $\boldsymbol{u}$ and the temperature $T$. The velocity and temperature are average properties of a collection of molecules and are related to the mass and velocity, $\boldsymbol{c}$, of the molecules.

The macroscopic velocity $\boldsymbol{u}$, defined as

$$
\boldsymbol{u} \equiv \bar{c},
$$


can be used to define the deviation of the molecular velocity at point $\boldsymbol{x}$ at time $t$ to the average velocity, known as the peculiar velocity:

$$
C \equiv c-u
$$

The temperature is related to the average kinetic energy of the molecules. The kinetic energy of a molecule is proportional to the square of the molecular velocity. Therefore, the average kinetic energy is related to the average of the squared molecular velocities, that is

$$
\overline{c^{2}}=u^{2}+2 \boldsymbol{u} \cdot \overline{\boldsymbol{C}}+\overline{C^{2}}=u^{2}+\overline{C^{2}} .
$$

The bulk kinetic energy of the molecules is the kinetic energy of the motion of the bulk $\frac{1}{2} m u^{2}$, and the energy related to the random motion of the molecules with respect to the the bulk velocity is proportional to $\overline{C^{2}}$. The temperature describes the average kinetic energy due to the random motion of the molecules with respect to the bulk velocity, therefore

$$
\frac{3}{2} k T=\frac{1}{2} m \overline{C^{2}}
$$

where $k$ is the Boltzmann constant.

Using the probability density function the macroscopic quantities are defined by integration over the velocity space:

$$
\begin{gathered}
n(\boldsymbol{x}, t) \equiv \int f(\boldsymbol{c} ; \boldsymbol{x}, t) \mathrm{d} \boldsymbol{c}, \\
\rho(\boldsymbol{x}, t) \equiv \int m f(\boldsymbol{c} ; \boldsymbol{x}, t) \mathrm{d} \boldsymbol{c}, \\
\rho(\boldsymbol{x}, t) \boldsymbol{u}(\boldsymbol{x}, t) \equiv \int m \boldsymbol{c} f(\boldsymbol{c} ; \boldsymbol{x}, t) \mathrm{d} \boldsymbol{c}, \\
\rho(\boldsymbol{x}, t) \frac{3 k T(\boldsymbol{x}, t)}{m} \equiv \int m\|\boldsymbol{c}-\boldsymbol{u}(\boldsymbol{x}, t)\|^{2} f(\boldsymbol{c} ; \boldsymbol{x}, t) \mathrm{d} \boldsymbol{c} . \\
\hline
\end{gathered}
$$

Generally, the macroscopic quantities can be obtained experimentally, while the microscopic quantities cannot. They are also important for equilibrium properties of the probability density function. 


\subsection{The Boltzmann equation}

The Boltzmann equation is a partial differential equation for the evolution in velocity, space and time of the probability density function $f(\boldsymbol{c} ; \boldsymbol{x}, t)$ for molecules with a specific velocity, subjected to forces and inter-molecular interactions. Chapman and Cowling (1991), [8], provide an extensive derivation of the Boltzmann equation including various aspects of specific approximations to the collision term. Boltzmann and Maxwell independently obtained equations for the probability density function [8]. The equation for the change of the probability density function $f(\boldsymbol{c}(t) ; \boldsymbol{x}(t), t)$ in time, subjected to a force field, and experiencing molecular interactions becomes

$$
f(\boldsymbol{c}(t+\delta t) ; \boldsymbol{x}(t+\delta t), t+\delta t)=f(\boldsymbol{c}(t) ; \boldsymbol{x}(t), t)+\delta_{\mathrm{e}} f .
$$

where $\delta_{\mathrm{e}} f$ is the effect of molecular interactions. Assuming that $f$ is a continuous function, division of equation (8.8) by $\delta t$ and taking the limit of $\delta t$ to zero leads to the differential equation in Lagrangian formulation

$$
\frac{\mathrm{d}}{\mathrm{d} t}(f(\boldsymbol{c}(t) ; \boldsymbol{x}(t), t))=\frac{\mathrm{d}_{\mathrm{e}} f}{\mathrm{~d} t} .
$$

The change in time for the location $\boldsymbol{x}$ and velocity $\boldsymbol{c}$ of a molecule with mass $m$ subjected to a force $\boldsymbol{F}$, without intermolecular interaction are

$$
\frac{\mathrm{d} \boldsymbol{x}}{\mathrm{d} t}=\boldsymbol{c}
$$

and

$$
m \frac{\mathrm{d} \boldsymbol{c}}{\mathrm{d} t}=\boldsymbol{F},
$$

respectively. Therefore, equation (8.9) can be written in terms of partial derivatives to time, space and the molecular velocity, that is

$$
\frac{\partial f}{\partial t}+\boldsymbol{c} \cdot \boldsymbol{\nabla}_{\boldsymbol{x}} f+\frac{\boldsymbol{F}}{m} \cdot \nabla_{\boldsymbol{c}} f=\frac{\partial_{\mathrm{e}} f}{\partial t},
$$

where $\boldsymbol{\nabla}_{\boldsymbol{x}}$ and $\boldsymbol{\nabla}_{\boldsymbol{c}}$ are the gradients in the spatial and velocity domain, respectively. Moreover, the force to which the molecule is subjected is due to the body force, $\frac{F}{m}=f$.

In cases of equilibrium, equilibrium distribution functions can be derived. The equilibrium distribution function $f^{(0)}$ depends on macroscopic quantities 
and is defined as

$$
f^{(0)}(\boldsymbol{c} ; \rho, \boldsymbol{u}, T) \equiv \frac{\rho}{m}\left(\frac{m}{2 \pi k T}\right)^{\frac{\mathcal{D}}{2}} e^{-\frac{m C^{2}}{2 k T}},
$$

with $\mathcal{D}$ the dimensionality of the space, and with $C^{2} \equiv\|\boldsymbol{c}-\boldsymbol{u}\|^{2}$.

\subsection{Approximation to the collision term}

The interaction term $\frac{\partial_{\mathrm{e}} f}{\partial t}$ describes the effect due to intermolecular forces. Calculating this interaction term involves integration over the entire spatial and velocity domain, which is unfeasible in case of a large number of molecules. Several methods exist to simplify the equation, in order to be able to approximate its solution for practical flow problems.

\subsubsection{BGK approximation}

To simplify the collision term, Bhatnagar, Gross and Krook, assumed that molecular interactions will force the probability density function to its local equilibrium $f^{(0)}(\boldsymbol{c} ; \rho, \boldsymbol{u}, T)$, the so-called BGK approximation. This results in an exponentially decaying difference between the probability density function and its local equilibrium, with a characteristic time $\tau$, leading to

$$
\left.\frac{\partial_{\mathrm{e}} f}{\partial t}\right|_{\mathrm{BGK}} \equiv-\frac{f-f^{(0)}}{\tau} .
$$

The time $\tau$ characterizes the time it takes for the local deviations from equilibrium to relax to the equilibrium state. This implies that for large $\tau$, deviations from the equilibrium state influence the fluid over a longer distance. For the macroscopic equations, these effects are translated into viscous and heat conduction effects; a higher viscosity, or a higher heat conductivity results in a larger area that experiences disturbances.

In the BGK approximation for purely viscous effects, kinematic viscosity $\nu$ and relaxation time $\tau_{\nu}$ are related by [8]

$$
\tau_{\nu}=\frac{m}{k T} \nu
$$


Moreover, for purely heat conduction effects, heat conductivity $\kappa$ and relaxation time $\tau_{\kappa}$ are related by [8]

$$
\tau_{\kappa}=\frac{3}{5} \frac{m}{k T} \frac{\kappa}{\rho c_{v}},
$$

with specific heat at constant volume $c_{v}$.

Therefore, this method is only exact if only one of the effects is present, or if relaxation time for viscous effects is equal to the relaxation time for conductive effects. In order to account for the presence of both viscous and heat conduction, some hybrid relaxation time should be determined. However, for this research, it is assumed that viscous effects largely dominate the heat conductive effects.

Note, that the equilibrium distribution function, as well as the relaxation time depends on macroscopic quantities. In this way, the BGK approach couples the differential equations as the macroscopic quantities are itself a function of the probability density function.

\subsubsection{BBGKY approximation}

Another approach to simplify the collision term is named after Bogoliubov, Born, Green, Kirkwood and Yvon [8]. Collisions force the probability density function to an equilibrium at a specific relaxation rate. However, when density increases, the chance of collision increases due to a decrease in effective volume. Moreover, short range intermolecular forces result in an effective correction on the equilibrium distribution function, while long range intermolecular forces result in an effective forcing. The resulting collision term $[24,8]$ becomes

$$
\begin{gathered}
\left.\frac{\partial_{e} f}{\partial t}\right|_{\text {BBGKY }}=\underbrace{\frac{\chi}{\tau}\left(f^{(0)}-f\right)}_{\text {Short range kinetic }}+\underbrace{b \rho \chi\left\{\boldsymbol { C } \cdot \left[\left(\frac{3}{2}-\frac{3 m C^{2}}{10 k T}\right) \boldsymbol{\nabla} \ln T\right.\right.}_{\text {Short range }} \\
\underbrace{\left.\left.-\boldsymbol{\nabla} \ln \left(\rho^{2} \chi T\right)\right]+\left(1-\frac{m C^{2}}{5 k T}\right) \boldsymbol{\nabla} \cdot \boldsymbol{u}-\frac{2}{5} \frac{m \boldsymbol{C C}}{k T}: \boldsymbol{\nabla}\right\} f^{(0)}}_{\text {Short range }}+\underbrace{\boldsymbol{\nabla} V_{m} \cdot \boldsymbol{\nabla}_{\boldsymbol{c}} f}_{\text {Long range }}
\end{gathered}
$$

where $b \rho$ represents the specific volume taken by the molecules, $\chi$ represents the increased collision probability due to that decrease in effective volume and 
$V_{m}$ represents the attractive force potential. Note that the first part, with a relaxation time to equilibrium, arises from a BGK approximation to one of the collision integrals in the exact approach.

Due to the increased collision probability, the relation of viscosity and relaxation time becomes [8]

$$
\frac{\tau_{\nu}}{\chi}\left\{1+\frac{4}{5} b \rho \chi+\left[\left(\frac{2}{5}\right)^{2}+\frac{3.007}{5}\right](b \rho \chi)^{2}\right\}=\frac{m}{k T} \nu
$$

and the relation of heat conduction and relaxation time becomes [8]

$$
\frac{\tau_{\kappa}}{\chi}\left\{1+\frac{6}{5} b \rho \chi+\left[\left(\frac{3}{5}\right)^{2}+0.6608 \times \frac{3.007}{5}\right](b \rho \chi)^{2}\right\}=\frac{3}{5} \frac{m}{k T} \frac{\kappa}{\rho c_{v}} .
$$

However, He \& Doolen (2002) report a different relation for viscosity and relaxation time [24]. In this research the relaxation time presented in equation (8.18) is used.

\subsection{Incorporating an equation of state for water}

The Boltzmann equation, with a specific collision term results in an equation of state for pressure as a function of density and temperature. However, this equation of state depends on the parameters in the equations. In this section, the derived Boltzmann equation and two approximations for the collision term are coupled to the known equation of state for water. For the BGK approach this will result in a volume force term, while the BBGKY approach includes short and long range intermolecular interactions through its parameters.

The pressure, resulting from the BGK approximation is for an ideal gas. For such a gas the pressure is related to the density and temperature by

$$
p_{\text {Ideal gas }}(\rho, T)=\rho \frac{k T}{m} .
$$

However, a dense gas or a liquid has a high loading of molecules which increases collision probability and leads to other short range interactions. Moreover, long range inter-molecular interactions cannot be neglected for such fluids. In literature, several models have been provided for various gases and liquids as a result of the type of molecular interactions included. For water, long 
range inter-molecular interactions are not only a result of Van der Waals forces, but are also a result of hydrogen bonds [26]. Jeffery \& Austin (1999), [26], provide an equation of state for water that incorporates short and long range inter-molecular interactions, which results in the near incompressibility that is typical for a liquid.

$$
p_{\mathrm{H}_{2} \mathrm{O}}(\rho, T)=\rho \frac{k T}{m}+\underbrace{\rho \frac{k T}{m} \frac{\alpha \rho}{1-\lambda b(T) \rho}}_{\text {Short range (repulsive) }}-\underbrace{\left(a_{\mathrm{VW}}+b^{*} \frac{k T}{m}\right) \rho^{2}}_{\text {Long range (attractive) }} .
$$

Note that a change in effective specific volume $b \rho$ is not only due to increased density, but also due to increased random motion, which is represented by the temperature dependency of $b$.

\subsubsection{BGK approximation}

Application of only the BGK approximation for molecular interaction results in the Navier-Stokes equations for an ideal gas. However, interest is in the NavierStokes equations for water. Therefore, a correction for the non-ideal gas effects is required. In this research, this correction is included by adding the non-ideal gas part of the pressure gradient to the body forces that are present, resulting in

$$
\frac{\boldsymbol{F}}{m}=\boldsymbol{f}-\frac{\boldsymbol{\nabla} p-\nabla p_{\text {Ideal gas }}}{\rho}
$$

In this approach the added pressure gradient term $\frac{\nabla p-\nabla p_{\text {Ideal gas }}}{\rho}$ is similar to the long range inter-molecular interaction term $\nabla V_{m}$ in equation (8.17). This approach treats both short and long range inter-molecular interactions as long range inter-molecular interactions. Moreover, the effect of velocity gradients for short range inter-molecular interactions will be neglected in this approach. This will influence both the rate at which equilibrium is reached as well as the magnitude of the observed deviations from equilibrium.

Substituting the pressure correction (8.22) in the Boltzmann equation (8.12) with the BGK collision term, using only gradients to density and temperature results in

$$
\begin{aligned}
& \frac{\partial f}{\partial t}+\boldsymbol{c} \cdot \boldsymbol{\nabla}_{\boldsymbol{x}} f \\
& \quad+\left[\boldsymbol{f}-\left(\frac{1}{\rho} \frac{\partial p}{\partial \rho}-\frac{k T}{\rho m}\right) \boldsymbol{\nabla} \rho-\left(\frac{1}{\rho} \frac{\partial p}{\partial T}-\frac{k}{m}\right) \boldsymbol{\nabla} T\right] \cdot \nabla_{\boldsymbol{c}} f=\frac{f^{(0)}-f}{\tau}
\end{aligned}
$$


Now substituting the equation of state for water provided by Jeffery \& Austin (1999) [26], equation (8.21), in equation (8.23) results in

$$
\begin{aligned}
& \frac{\partial f}{\partial t}+\boldsymbol{c} \cdot \nabla_{\boldsymbol{x}} f+\left[\boldsymbol{f}-\left(\frac{k T}{m} \alpha \frac{2-\lambda b \rho}{(1-\lambda b \rho)^{2}}-2\left(a_{\mathrm{VW}}+b^{*} \frac{k T}{m}\right)\right) \nabla \rho\right. \\
& \left.-\left(\frac{k}{m} \alpha \rho \frac{1+\lambda b \rho\left(\frac{T}{b} \frac{\mathrm{d} b}{\mathrm{~d} T}-1\right)}{(1-\lambda b \rho)^{2}}-b^{*} \frac{k}{m} \rho\right) \nabla T\right] \cdot \nabla_{\boldsymbol{c}} f=\frac{1}{\tau}\left(f^{(0)}-f\right) .
\end{aligned}
$$

\subsubsection{BBGKY approximation}

The BBGKY approximation for which the collision term is provided in equation (8.17) results in the following expression for the pressure of dense single phase gases [24]

$$
p=\rho \frac{k T}{m}+\underbrace{b \rho^{2} \chi \frac{k T}{m}}_{\mathrm{SR}}-\underbrace{a \rho^{2}}_{\mathrm{LR}}
$$

where the long range part is related to the attractive force potential $V_{m}$ by

$$
\nabla V_{m}=-\nabla a \rho^{2}
$$

Substituting the long range part of equation (8.21), this implies that the gradient of the attractive force potential for water is

$$
\nabla V_{m, \mathrm{H}_{2} \mathrm{O}}=-2\left(a_{\mathrm{VW}}+b^{*} \frac{k T}{m}\right) \nabla \rho-b^{*} \frac{k T}{m} \rho \frac{\nabla T}{T} .
$$

Furthermore, it also implies that

$$
[b \rho \chi]_{\mathrm{H}_{2} \mathrm{O}}=\frac{\alpha \rho}{1-\lambda b(T) \rho} .
$$

Substituting $b_{\mathrm{H}_{2} \mathrm{O}} \equiv b(T)$ results in

$$
\chi_{\mathrm{H}_{2} \mathrm{O}}=\frac{\alpha}{b(T)} \frac{1}{1-\lambda b(T) \rho} .
$$

As a result the BBGKY approximation for the Boltzmann equation for water 
becomes

$$
\begin{aligned}
\frac{\partial f}{\partial t}+\boldsymbol{c} \cdot \boldsymbol{\nabla}_{\boldsymbol{x}} f+\boldsymbol{f} \cdot \boldsymbol{\nabla}_{\boldsymbol{c}} f=\frac{\chi}{\tau}\left(f^{(0)}-f\right) \\
+b \rho \chi\left\{\frac { m \boldsymbol { C } } { k T } \cdot \left[\left(\frac{3 k T}{2 m}-\frac{3 C^{2}}{10}\right) \frac{\boldsymbol{\nabla} T}{T}+\frac{k T b \chi}{m \alpha \rho}(\lambda b \rho-2) \boldsymbol{\nabla} \rho\right.\right. \\
\left.+\frac{k T b \chi}{m \alpha}\left(\lambda b \rho\left(1-2 \frac{T}{b} \frac{\mathrm{d} b}{\mathrm{~d} T}\right)+\frac{T}{b} \frac{\mathrm{d} b}{\mathrm{~d} T}-1\right) \frac{\boldsymbol{\nabla} T}{T}\right] \\
\left.+\left(1-\frac{m C^{2}}{5 k T}\right) \boldsymbol{\nabla} \cdot \boldsymbol{u}-\frac{2}{5} \frac{m \boldsymbol{C} \boldsymbol{C}}{k T}: \boldsymbol{\nabla} \boldsymbol{u}\right\} f^{(0)} \\
-\left\{2\left(a_{\mathrm{VW}}+b^{*} \frac{k T}{m}\right) \boldsymbol{\nabla} \rho+b^{*} \frac{k T}{m} \rho \frac{\boldsymbol{\nabla} T}{T}\right\} \cdot \nabla_{\boldsymbol{c}} f .
\end{aligned}
$$

It can be observed that the attractive part in the BBGKY model is equal to the attractive part in the pressure corrected BGK formulation. However, the short range repulsive forces not only have a contribution through the gradients of the density and temperature, but also have a contribution through gradients in the velocity. Moreover, the nature of the short range interactions has changed from a volumetric force field in the pressure corrected BGK to a source term in the BBGKY model.

\subsubsection{Viscosity of water}

Both models require the kinematic viscosity $\nu$ to couple the relaxation time to the macroscopic quantities. The dynamic viscosity $\mu$ is related to the kinematic viscosity by density

$$
\nu \equiv \frac{\mu}{\rho}
$$

Several theoretical and experimental expressions exist for the dynamic viscosity of fluids and an extensive discussion of them can be found in literature, e.g. by Touloukian et al. (1975) [38].

The dynamic viscosity as a function of density and temperature is usually expressed in terms of the reduced density and reduced temperature. The reduced quantities are the actual quantities divided by their values at the critical point, namely

$$
\rho_{\mathrm{R}} \equiv \frac{\rho}{\rho_{\mathrm{C}}} ; \quad T_{\mathrm{R}} \equiv \frac{T}{T_{\mathrm{C}}} .
$$


Liquid water is nearly incompressible. Therefore, as a first approximation, viscosity can be considered to be a function of temperature only. A recommended correlation [38], used in this research is

$$
\mu\left(T_{\mathrm{R}}\right)=a 10^{\frac{b}{T_{\mathrm{R}}^{-c}}},
$$

with $a, b$ and $c$ empirical dimensionless parameters that have been determined with experimental data. For the viscosity of water, it is found that [38]

$$
\begin{array}{rll}
a & 2.414 \times 10^{-5} & {\left[\mathrm{~kg} \mathrm{~m}^{-1} \mathrm{~s}^{-1}\right]} \\
b T_{\mathrm{C}} & 2.478 \times 10^{2} & {[\mathrm{~K}]} \\
c T_{\mathrm{C}} & 1.40 \times 10^{2} & {[\mathrm{~K}]}
\end{array}
$$

Table 8.1: Empirical parameters for the viscosity of water in equation (8.33) recommended by Touloukian et al. (1975) [38]

Note that the value of the critical temperature $T_{\mathrm{C}}$ is not necessary for evaluation of $\mu$ and is not discussed here.

\subsection{Approximation for $\nabla_{c} f$}

The derivative with respect to molecular velocity of the distribution function introduces a complexity. When a proper discretization of velocity space has been chosen, a finite set of discrete values of the distribution functions is known. This finite set would not be sufficient to obtain accurate derivatives in velocity space. The shift of the distribution function in velocity space under influence of forces has to be taken into account, or the derivative has to be approximated. As a shift in velocity space increases the complexity of the problem significantly an approximation to the derivatives in velocity space has been chosen. It is assumed that deviations from the equilibrium distribution will be such that the derivatives of the distribution function in velocity space can be approximated by the derivatives of the equilibrium distribution function [24]

$$
\nabla_{c} f \approx \nabla_{c} f^{(0)}
$$

Substitution of the equilibrium distribution function, equation (8.13) results in

$$
\nabla_{c} f \approx-\frac{m \boldsymbol{C}}{k T} f^{(0)}
$$


The BGK approximation with a pressure correction will show many similarities to the BBGKY approximation using this approximation to the equilibrium distribution function.

\subsubsection{BGK approximation for water}

Substituting the approximation for the derivative with respect to molecular velocity of the distribution function (8.35) in equation (8.24) leads to

$$
\begin{aligned}
& \frac{\partial f}{\partial t}+\boldsymbol{c} \cdot \boldsymbol{\nabla}_{\boldsymbol{x}} f= \frac{k T}{m \nu}\left(f^{(0)}-f\right) \\
&+\frac{m \boldsymbol{C}}{k T} \cdot\left\{\boldsymbol{f}-\left[\frac{k T}{m} \alpha \frac{2-\lambda b \rho}{(1-\lambda b \rho)^{2}}-2\left(a_{\mathrm{VW}}+b^{*} \frac{k T}{m}\right)\right] \boldsymbol{\nabla} \rho\right. \\
&\left.-\left[\frac{k}{m} \alpha \rho \frac{1+\lambda b \rho\left(\frac{T}{b} \frac{\mathrm{d} b}{\mathrm{~d} T}-1\right)}{(1-\lambda b \rho)^{2}}-b^{*} \frac{k}{m} \rho\right] \nabla T\right\} f^{(0)}
\end{aligned}
$$

This is written in a more compact form as

$$
\frac{\partial f}{\partial t}+\boldsymbol{c} \cdot \nabla_{\boldsymbol{x}} f=\frac{k T}{m \nu}\left(f^{(0)}-f\right)+\frac{m \boldsymbol{C}}{k T} \cdot\left\{\boldsymbol{f}+\mathcal{H}_{\rho} \boldsymbol{\nabla} \rho+\mathcal{H}_{T} \boldsymbol{\nabla} T\right\} f^{(0)},
$$

with $\mathcal{H}_{\rho}$ and $\mathcal{H}_{T}$ the functions multiplying the density gradient and temperature gradient part of the pressure correction, respectively. These are expressed as

$$
\begin{gathered}
\mathcal{H}_{\rho}=\frac{k T}{m} \alpha \frac{\lambda b \rho-2}{(1-\lambda b \rho)^{2}}+2\left(a_{\mathrm{VW}}+b^{*} \frac{k T}{m}\right), \\
\mathcal{H}_{T}=\frac{k}{m} \alpha \rho \frac{\lambda b \rho\left(1-\frac{T}{b} \frac{\mathrm{d} b}{\mathrm{~d} T}\right)-1}{(1-\lambda b \rho)^{2}}+b^{*} \frac{k}{m} \rho .
\end{gathered}
$$

\subsubsection{BBGKY approximation for water}

Substituting the approximation for the derivative with respect to molecular velocity of the distribution functions in velocity space (8.35) in equation (8.30) 
leads to

$$
\begin{aligned}
& \frac{\partial f}{\partial t}+\boldsymbol{c} \cdot \boldsymbol{\nabla}_{\boldsymbol{x}} f=\frac{\chi}{\tau}\left(f^{(0)}-f\right) \\
&+\frac{m \boldsymbol{C}}{k T} \cdot\left\{\boldsymbol{f}+2\left(a_{\mathrm{VW}}+b^{*} \frac{k T}{m}\right) \boldsymbol{\nabla} \rho+b^{*} \frac{k T}{m} \rho \frac{\boldsymbol{\nabla} T}{T}\right. \\
&+\left[b \rho \chi\left(\frac{3 k T}{2 m}-\frac{3 C^{2}}{10}\right) \frac{\boldsymbol{\nabla} T}{T}+\frac{k T}{m} \alpha \frac{\lambda b \rho-2}{(1-\lambda b \rho)^{2}} \boldsymbol{\nabla} \rho\right. \\
&+\frac{k T}{m} \alpha \rho \frac{\lambda b \rho\left(1-2 \frac{T}{b} \frac{\mathrm{d} b}{\mathrm{~d} T}\right)+\frac{T}{b} \frac{\mathrm{d} b}{\mathrm{~d} T}-1}{(1-\lambda b \rho)^{2}} \frac{\boldsymbol{\nabla} T}{T} \\
&\left.\left.-b \rho \chi \frac{1}{5} \boldsymbol{C} \boldsymbol{\nabla} \cdot \boldsymbol{u}-b \rho \chi \frac{2}{5} \boldsymbol{C} \cdot \boldsymbol{\nabla} \boldsymbol{u}\right]\right\} f^{(0)}+b \rho \chi \boldsymbol{\nabla} \cdot \boldsymbol{u} f^{(0)}
\end{aligned}
$$

Similar to the BGK formulation this is written in more compact form, while $\frac{\chi}{\tau}$ has been replaced by its viscosity dependency (8.18)

$$
\begin{aligned}
\frac{\partial f}{\partial t}+\boldsymbol{c} \cdot \boldsymbol{\nabla}_{\boldsymbol{x}} f=\left\{1+\frac{4}{5} \frac{\alpha \rho}{1-\lambda b \rho}+\frac{19.035(\alpha \rho)^{2}}{25(1-\lambda b \rho)^{2}}\right\} \frac{k T}{m \nu}\left(f^{(0)}-f\right) \\
+\frac{\alpha \rho}{1-\lambda b \rho} \boldsymbol{\nabla} \cdot \boldsymbol{u} f^{(0)}+\frac{m \boldsymbol{C}}{k T} \cdot\left\{\boldsymbol{f}+\mathcal{H}_{\rho} \boldsymbol{\nabla} \rho+\mathcal{H}_{T} \boldsymbol{\nabla} T-\mathcal{H}_{\boldsymbol{u}}\right\} f^{(0)}
\end{aligned}
$$

with $\mathcal{H}_{\rho}$ and $\mathcal{H}_{T}$ the functions multiplying the density gradient and temperature gradient part of the attractive and repulsive molecular interactions, respectively and $\mathcal{H}_{\boldsymbol{u}}$ the vector due to gradients in the velocity of the attractive and repulsive molecular interactions. These are expressed as

$$
\begin{array}{r}
\mathcal{H}_{\rho}=\frac{k T}{m} \alpha \frac{\lambda b \rho-2}{(1-\lambda b \rho)^{2}}+2\left(a_{\mathrm{VW}}+b^{*} \frac{k T}{m}\right), \\
\mathcal{H}_{T}=\frac{k}{m} \rho\left\{\alpha \frac{\lambda b \rho\left(1-2 \frac{T}{b} \frac{\mathrm{d} b}{\mathrm{~d} T}\right)+\frac{T}{b} \frac{\mathrm{d} b}{\mathrm{~d} T}-1}{(1-\lambda b \rho)^{2}}+b^{*}\right. \\
\left.+\frac{\alpha}{1-\lambda b \rho}\left(\frac{3}{2}-\frac{3 m C^{2}}{10 k T}\right)\right\}, \\
\mathcal{H}_{\boldsymbol{u}}=\frac{\alpha \rho}{1-\lambda b \rho}\left(\frac{\boldsymbol{C}}{5} \boldsymbol{\nabla} \cdot \boldsymbol{u}+\frac{2}{5} \boldsymbol{C} \cdot \boldsymbol{\nabla} \boldsymbol{u}\right) .
\end{array}
$$




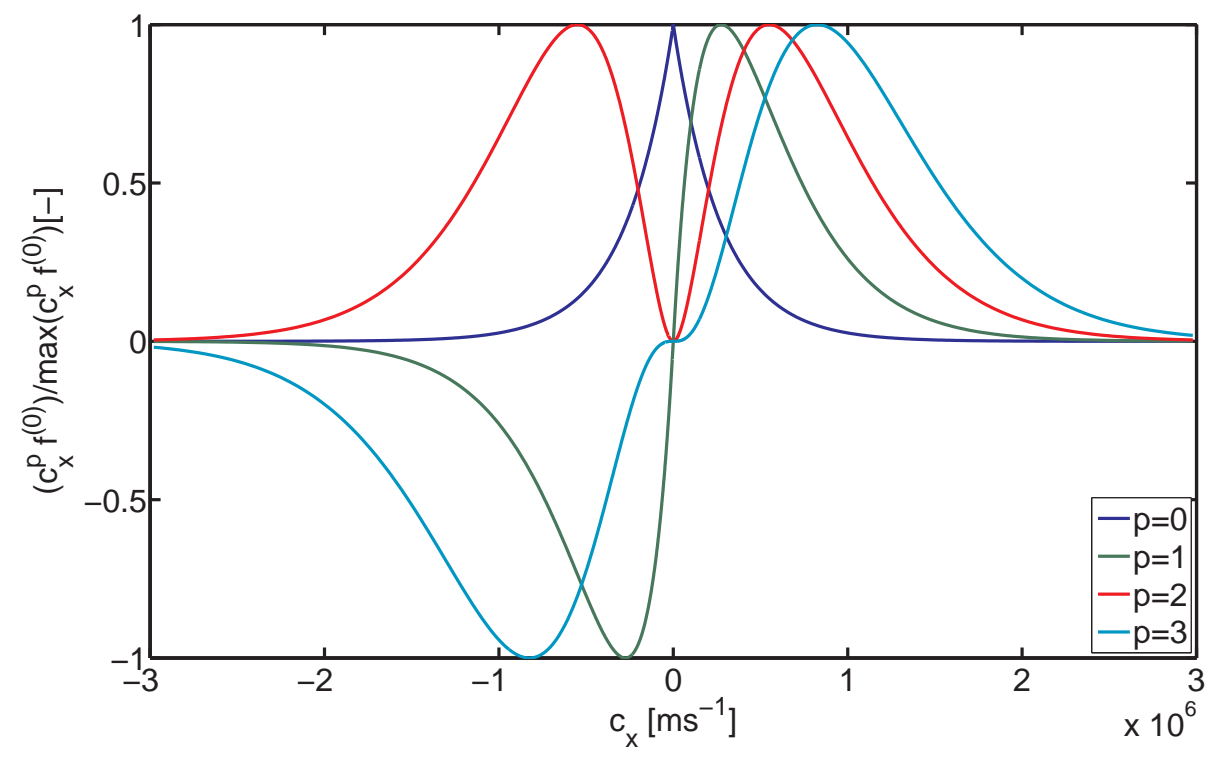

Figure 8.1: $\boldsymbol{c}^{p} f^{(0)}$ for $u=0$, normalized by its maximum for the first $p^{\text {th }}$ moments in one dimension. Density, velocity and temperature require up to the $0^{\text {th }}, 1^{\text {st }}$ and $2^{\text {nd }}$ moment, respectively.

Comparing the BGK approach to the BBGKY approach, it becomes clear that both approaches lead to similar equations. The contribution due to the density gradient is exactly the same. The BBGKY approach leads to a slightly different relaxation rate, and has a slightly different contribution related to the temperature gradient. Moreover, contrary to the BGK approach, in the BBGKY formulation the gradient and the divergence of the macroscopic velocity has an influence.

\subsection{Discretized velocity space}

For fluid flow problems, the solution space can usually be reduced using a bounded space in which the important phenomena take place, and include far field phenomena using appropriate boundary conditions. However, in the Boltz- 
mann approach, the velocity solution space is not easily reduced to a limited range of significant velocities. Figure 8.1 shows the functions that need to be integrated in order to obtain moments of the equilibrium distribution, $c^{p} f^{(0)}$. It can be observed that with increasing $p$ the velocity at which the peak is observed increases in value, while the range of velocities with significant values of the distribution function increases.

Due to the nature of the equilibrium distribution function, moments of that function can be evaluated exactly requiring a limited number of velocities using Gauß-Hermite quadrature. For an arbitrary $n^{\text {th }}$-order polynomial $p_{n}(\boldsymbol{c})$, a change of variables is used in order to be able to use the quadrature

$$
\begin{aligned}
\int p_{n} & (\boldsymbol{c}) \frac{\rho}{m}\left(\frac{m}{2 \pi k T}\right)^{\frac{\mathcal{D}}{2}} e^{-\frac{m C^{2}}{2 k T}} \mathrm{~d} \boldsymbol{c} \\
= & \frac{\rho}{m} \pi^{-\frac{\mathcal{D}}{2}} \int p_{n}\left(\boldsymbol{\xi} \sqrt{\frac{2 k T}{m}}+\boldsymbol{u}\right) e^{-\xi^{2}} \mathrm{~d} \boldsymbol{\xi},
\end{aligned}
$$

with normalized peculiar velocity

$$
\boldsymbol{\xi} \equiv \boldsymbol{C} \sqrt{\frac{m}{2 k T}}
$$

Subsequently, a Gauß-Hermite quadrature leads to

$$
\int p_{n}(\boldsymbol{c}) f^{(0)}(\boldsymbol{c}) \mathrm{d} \boldsymbol{c}=\frac{\rho}{m} \pi^{-\frac{\mathcal{D}}{2}} \sum_{\imath} \omega_{\imath} p_{n}\left(\boldsymbol{\xi}_{\imath} \sqrt{\frac{2 k T}{m}}+\boldsymbol{u}\right),
$$

where $\omega_{\imath}$ and $\boldsymbol{\xi}_{\imath}$ are the weights and normalized peculiar velocities required for the desired order of the polynomial. For a polynomial of order $n, \frac{n+1}{2}$ points are required in each dimension in order to obtain an exact solution. This means that if $n=5$, a total of $3^{\mathcal{D}}$ points are required to obtain an exact solution. However, practical application of the Gauß-Hermite quadrature requires it to be applied to the actual distribution function. Approximating the ratio of the distribution function and the equilibrium distribution as a polynomial, the quadrature can be applied, given a high enough order of the quadrature. This results in

$$
\int p_{n}(\boldsymbol{c}) f(\boldsymbol{c}) \mathrm{d} \boldsymbol{c} \approx \frac{\rho}{m} \pi^{-\frac{\mathcal{D}}{2}} \sum_{\imath} \omega_{\imath} p_{n}\left(\boldsymbol{\xi}_{\imath} \sqrt{\frac{2 k T}{m}}+\boldsymbol{u}\right) \frac{f\left(\boldsymbol{c}_{\imath}\right)}{f^{(0)}\left(\boldsymbol{c}_{\imath}\right)} .
$$

Moreover, still a multitude of discrete values of the distribution function would be necessary, as the nodes of the Gauß-Hermite quadrature fix the peculiar velocity and not the molecular velocity itself. 
The classical Lattice Boltzmann approach splits the term $e^{\frac{m c^{2}}{2 k T}}$ from the exponent $e^{\frac{m C^{2}}{2 k T}}$ in the equilibrium distribution, and approximates the remainder of the distribution function with a Taylor series expansion. For the isothermal case the quadrature can then be applied to $\boldsymbol{\xi}_{\imath, 0}=\boldsymbol{c}_{\imath} \sqrt{\frac{m}{2 k T}}$. If thermal effects need to be taken into account, also the non-isothermal part should be split for this approach. Note, that this approach increases the order of the polynomial function by the used order of the Taylor series expansion, which increases the number of points required for the quadrature.

In contrast to the classical approach, we propose to approximate the difference between the actually required distribution function and a distribution function with constant $c_{\imath, 0}$ by

$$
f\left(\boldsymbol{c}_{\imath}\right)-f\left(\boldsymbol{c}_{\imath, 0}\right) \approx f^{(0)}\left(\boldsymbol{c}_{\imath}\right)-f^{(0)}\left(\boldsymbol{c}_{\imath, 0}\right) .
$$

This approach is based on the assumption that $\nabla_{c} f \approx \nabla_{c} f^{(0)}$, and as a result

$$
\int_{c_{0}}^{c} \nabla_{\boldsymbol{c}} f \mathrm{~d} \boldsymbol{c} \approx \int_{\boldsymbol{c}_{0}}^{\boldsymbol{c}} \nabla_{\boldsymbol{c}} f^{(0)} \mathrm{d} \boldsymbol{c}
$$

for sufficiently small deviations of $f(\boldsymbol{c})$ from the equilibrium distribution function $f^{(0)}(\boldsymbol{c})$. The result of this approach is a non-linear system of algebraic equations for the density, velocity and temperature

$$
\begin{gathered}
\rho: \quad 0=\sum_{\imath} m w_{\imath} \frac{f_{\imath, 0}-f_{\imath, 0}^{(0)}}{f_{\imath}^{(0)}}, \\
\rho \boldsymbol{u}: \quad \mathbf{0}=\sum_{\imath} m w_{\imath} \boldsymbol{C}_{\imath} \frac{f_{\imath, 0}-f_{\imath, 0}^{(0)}}{f_{\imath}^{(0)}}, \\
\rho \frac{3 k T}{m}: \quad 0=\sum_{\imath} m w_{\imath}\left\|\boldsymbol{C}_{\imath}\right\|^{2} \frac{f_{\imath, 0}-f_{\imath, 0}^{(0)}}{f_{\imath}^{(0)}},
\end{gathered}
$$

where

$$
f_{\imath} \equiv f\left(\boldsymbol{c}_{\imath}\right) ; \quad f_{\imath, 0} \equiv f\left(\boldsymbol{c}_{\imath, 0}\right) ; \quad w_{\imath} \equiv \frac{\omega_{\imath}}{\pi^{\frac{\mathcal{D}}{2}}}
$$




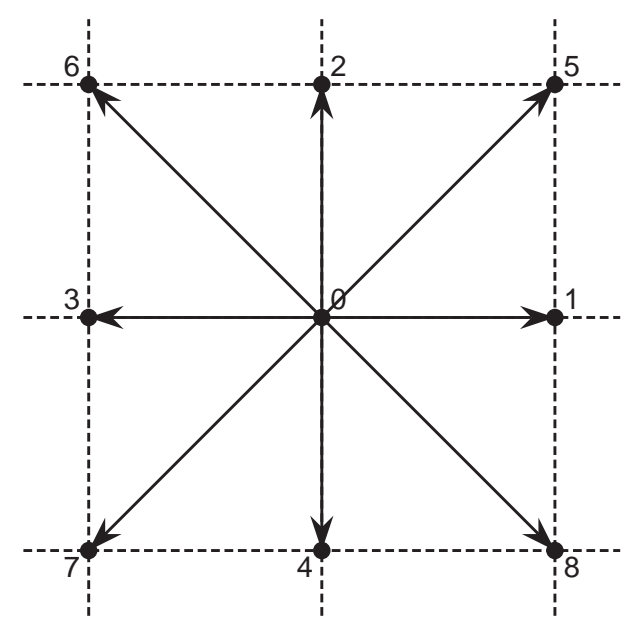

Figure 8.2: Velocity vectors used for a three-point Gauß-Hermite quadrature in two dimensions, leading to the D2Q9 stencil.

For a three-point Gauß-Hermite quadrature in one dimension, often called the D1Q3 stencil, the required velocities and corresponding weights are

$$
\begin{gathered}
\boldsymbol{C}_{\mathrm{D} 1 \mathrm{Q} 3}^{\mathrm{T}} \equiv\left[\begin{array}{lll}
C_{-1} & C_{0} & C_{+1}
\end{array}\right]=\sqrt{\overline{\overline{C^{2}}}}\left[\begin{array}{lll}
-1 & 0 & 1
\end{array}\right] \\
\boldsymbol{w}_{\mathrm{D} 1 \mathrm{Q} 3}^{\mathrm{T}} \equiv\left[\begin{array}{lll}
w_{C_{-1}} & w_{C_{0}} & w_{C_{+1}}
\end{array}\right]=\frac{1}{6}\left[\begin{array}{lll}
1 & 4 & 1
\end{array}\right] .
\end{gathered}
$$

Extension of the three-point Gauß-Hermite quadrature to two dimensions results in the D2Q9 stencil. The corresponding nodes, and weights, constructed from the 1-D velocities and weights, are

$$
\left[\begin{array}{lll}
\boldsymbol{C}_{7} & \boldsymbol{C}_{4} & \boldsymbol{C}_{8} \\
\boldsymbol{C}_{3} & \boldsymbol{C}_{0} & \boldsymbol{C}_{1} \\
\boldsymbol{C}_{6} & \boldsymbol{C}_{2} & \boldsymbol{C}_{5}
\end{array}\right]_{\mathrm{D} 2 \mathrm{Q} 9} \equiv\left[\begin{array}{lll}
\left(\begin{array}{l}
C_{-1} \\
C_{-1}
\end{array}\right) & \left(\begin{array}{l}
C_{0} \\
C_{-1}
\end{array}\right) & \left(\begin{array}{l}
C_{+1} \\
C_{-1}
\end{array}\right) \\
\left(\begin{array}{l}
C_{-1} \\
C_{0}
\end{array}\right) & \left(\begin{array}{l}
C_{0} \\
C_{0}
\end{array}\right) & \left(\begin{array}{l}
C_{+1} \\
C_{0}
\end{array}\right) \\
\left(\begin{array}{l}
C_{-1} \\
C_{+1}
\end{array}\right) & \left(\begin{array}{l}
C_{0} \\
C_{+1}
\end{array}\right) & \left(\begin{array}{l}
C_{+1} \\
C_{+1}
\end{array}\right)
\end{array}\right],
$$




$$
\left[\begin{array}{lll}
w_{\boldsymbol{C}_{7}} & w_{\boldsymbol{C}_{4}} & w_{\boldsymbol{C}_{8}} \\
w_{\boldsymbol{C}_{3}} & w_{\boldsymbol{C}_{0}} & w_{\boldsymbol{C}_{1}} \\
w_{\boldsymbol{C}_{6}} & w_{\boldsymbol{C}_{2}} & w_{\boldsymbol{C}_{5}}
\end{array}\right]_{\mathrm{D} 2 \mathrm{Q} 9} \equiv \boldsymbol{w}_{\mathrm{D} 1 \mathrm{Q} 3} \boldsymbol{w}_{\mathrm{D} 1 \mathrm{Q} 3}
$$

The resulting lattice is presented in figure 8.2.

In a similar way the D3Q27 can be constructed from the D1Q3 stencil applied to all dimensions.

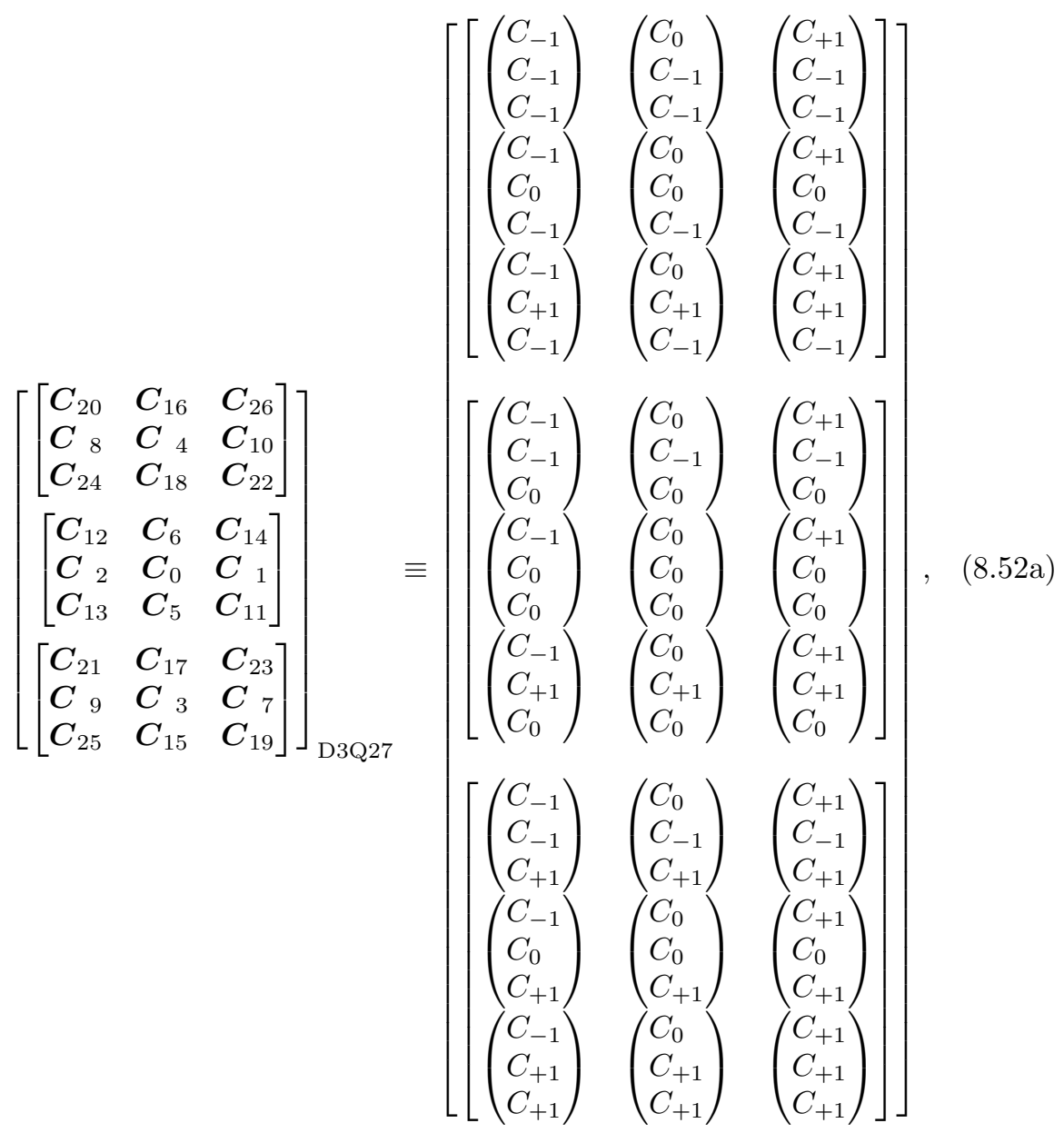




$$
\left[\begin{array}{lll}
{\left[\begin{array}{lll}
w_{\boldsymbol{C}_{20}} & w_{\boldsymbol{C}_{16}} & w_{\boldsymbol{C}_{26}} \\
w_{\boldsymbol{C}} & w_{\boldsymbol{C}} & w_{\boldsymbol{C}_{10}} \\
w_{\boldsymbol{C}_{24}} & w_{\boldsymbol{C}_{18}} & w_{\boldsymbol{C}_{22}}
\end{array}\right]} \\
{\left[\begin{array}{lll}
w_{\boldsymbol{C}_{12}} & w_{\boldsymbol{C}_{6}} & w_{\boldsymbol{C}_{14}} \\
w_{\boldsymbol{C}_{2}} & w_{\boldsymbol{C}_{0}} & w_{\boldsymbol{C}_{1}} \\
w_{\boldsymbol{C}_{13}} & w_{\boldsymbol{C}_{5}} & w_{\boldsymbol{C}_{11}}
\end{array}\right]} \\
{\left[\begin{array}{lll}
w_{\boldsymbol{C}_{21}} & w_{\boldsymbol{C}_{17}} & w_{\boldsymbol{C}_{23}} \\
w_{\boldsymbol{C}} & w_{\boldsymbol{C}} & w_{\boldsymbol{C}_{7}} \\
w_{\boldsymbol{C}_{25}} & w_{\boldsymbol{C}_{15}} & w_{\boldsymbol{C}_{19}}
\end{array}\right]}
\end{array}\right]_{\mathrm{D} 3 \mathrm{Q} 27} \equiv \boldsymbol{w}_{\mathrm{D} 1 \mathrm{Q} 3} \otimes\left[\boldsymbol{w}_{\mathrm{D} 1 \mathrm{Q} 3} \boldsymbol{w}_{\mathrm{D} 1 \mathrm{Q} 3}\right]
$$

with the Kronecker product $\otimes$. Moreover, for isothermal systems, the order of accuracy can be relaxed, leading to the D3Q15 and D3Q19 stencils. These stencils redistribute the influence of specific velocity directions over the remaining velocity directions.

The velocities for which the Boltzmann equation will be solved, can then be written as

$$
\boldsymbol{c}_{\imath, 0}=\frac{\boldsymbol{C}_{\imath, 0}}{\sqrt{\theta_{0}}}+\boldsymbol{u}_{0}-\boldsymbol{U},
$$

with $C_{\imath, 0}$ the initial peculiar velocity required for the quadrature, $u_{0}$ the initial average velocity, $\theta_{0}$ the initial relative temperature and $\boldsymbol{U}$ a constant offset. The relative temperature is defined as

$$
\theta \equiv \frac{T}{T_{\mathrm{r}}} .
$$

The relative temperature $T_{\mathrm{r}}$ should be chosen such that the used velocities are close to the required peculiar velocities for the quadrature. A good candidate is the expected average temperature, but also the initial average temperature can be considered, for instance in the case of near isothermal flows. For $\boldsymbol{U}$, the choice will be affected by the space-time discretization, introduced in the next section.

\subsection{Space-time lattice discretization}

Consider the general Boltzmann equation (8.12) for velocity $\boldsymbol{c}_{\imath, 0}$. The equation can be evaluated along its characteristic $\boldsymbol{c}_{\imath, 0}$, leading to

$$
\left.\frac{\partial f}{\partial t}\right|_{\boldsymbol{x}=\boldsymbol{c}_{\imath, 0} t}+\frac{\boldsymbol{F}}{m} \cdot \nabla_{\boldsymbol{c}} f=\left.\frac{\partial_{\mathrm{e}} f}{\partial t}\right|_{\boldsymbol{x}=\boldsymbol{c}_{\imath, 0} t},
$$


with the characteristic curve defined by

$$
\frac{\mathrm{d} \boldsymbol{x}}{\mathrm{d} t}=\boldsymbol{c}_{\imath, 0}
$$

In figure 8.2, and equations (8.50a), (8.51a), (8.52a) and (8.53), it can be observed that characteristics are always $U_{0, i}$ or $U_{0, i} \pm \sqrt{\frac{\overline{C_{0}^{2}}}{\theta_{0}}}$ in each of the dimensions $i$. For a grid moving with constant velocity $\boldsymbol{U}_{0}$, the spacing $\Delta x$ and time-step $\Delta t$ can be coupled by

$$
\Delta x=\Delta t \sqrt{\overline{C_{\mathrm{r}}^{2}}}
$$

with the lattice speed defined by

$$
\sqrt{\overline{C_{\mathrm{r}}^{2}}} \equiv \sqrt{\frac{\overline{C_{0}^{2}}}{\theta_{\mathrm{r}}}} .
$$

In the standard Lattice Boltzmann Method [24], this space-time coupling is exploited in order to obtain a collision and a streaming phase. Moreover, a second order accurate semi-explicit time-discretization is applied in the standard Lattice Boltzmann Method. The semi-explicit time-discretization is based on a Crank-Nicholson scheme. The assumption that the macroscopic quantities are slowly varying in time with respect to the distribution function and can be considered constant during each time-step results in an explicit scheme with an adapted relaxation time. In such a case the discrete dimensionless relaxation time and actual relaxation time are related by $\hat{\tau}=\frac{\tau}{\Delta t}+\frac{1}{2}$. This results in an often reported LBM viscosity of $\nu_{\mathrm{LBM}}=\left(\hat{\tau}-\frac{1}{2}\right) \overline{C_{\mathrm{r}}^{2}} \Delta t$, e.g. [9]. Note that the assumption of slowly varying macroscopic properties is only valid for grids with sufficient resolution, as macroscopic quantities should be slowly varying in time along the characteristic of the considered lattice velocity.

For an ideal-gas, the speed of sound is equal to the lattice speed, and stability and convergence rate of the solution will be dominated by the relaxation time and flow characteristics such as the Reynolds number. Morover, for the standard Lattice Boltzmann Method stability will also be determined by the validity of the assumption of slow variations of the macroscopic quantities. However, for non-ideal gases, the speed of sound and the lattice speed will not be equal, and stability and convergence rate of the solution will also be influenced by the speed of sound. In order to ensure stability and increased convergence rate, the space 
and time discretization have been decoupled by a parameter $\gamma$, which is defined as

$$
\gamma \equiv \frac{\Delta t \sqrt{\overline{C_{\mathrm{r}}^{2}}}}{\Delta x} .
$$

Generally, $\gamma$ will be smaller than one in order to ensure stability. A CrankNicholson scheme will be applied to the Boltzmann equation, and its discretization for both the pressure corrected BGK method and the BBGKY method with BGK approximation for the standard collision term can be written as

$$
\begin{aligned}
\left\{f_{\imath}-\frac{\Delta t}{2}\left[\frac{\chi}{\tau}\right.\right. & \left.\left.\left(f_{\imath}^{(0)}-f_{\imath}\right)+\mathcal{F}_{\imath} f_{\imath}^{(0)}\right]\right\}_{\boldsymbol{x}}^{t} \\
& =\left\{f_{\imath}+\frac{\Delta t}{2}\left[\frac{\chi}{\tau}\left(f_{\imath}^{(0)}-f_{\imath}\right)+\mathcal{F}_{\imath} f_{\imath}^{(0)}\right]\right\}_{\boldsymbol{x}-\Delta t \boldsymbol{c}_{\imath}}^{t-\Delta t}
\end{aligned}
$$

where $\mathcal{F}_{\imath}$ is the contribution of volumetric forces and non-ideal intermolecular interactions. In this research, the value of the right hand side contribution at $\boldsymbol{x}-\Delta t \boldsymbol{c}_{\imath}$ is obtained by linear interpolation with the values in $\boldsymbol{x}-\frac{\text { floor } \gamma}{\gamma} \Delta t \boldsymbol{c}_{\imath}$, and $\boldsymbol{x}-\frac{\text { ceil } \gamma}{\gamma} \Delta t \boldsymbol{c}_{\imath}$, where floor provides the nearest integer value equal to or smaller than $\gamma$ and ceil provides the nearest integer value equal to or larger than $\gamma$, respectively. In other words the value is obtained by linear interpolation in the direction of the considered lattice between the nearest points, resulting in

$$
\begin{aligned}
\left\{f_{\imath}\right. & \left.-\frac{\Delta t}{2}\left[\frac{\chi}{\tau}\left(f_{\imath}^{(0)}-f_{\imath}\right)+\mathcal{F}_{\imath} f_{\imath}^{(0)}\right]\right\}_{x}^{t} \\
= & (\gamma-\text { floor } \gamma)\left\{f_{\imath}+\frac{\Delta t}{2}\left[\frac{\chi}{\tau}\left(f_{\imath}^{(0)}-f_{\imath}\right)+\mathcal{F}_{\imath} f_{\imath}^{(0)}\right]\right\}_{\boldsymbol{x}-\frac{\text { ceil } \gamma}{\gamma} \Delta t \boldsymbol{c}_{\imath}}^{t-\Delta t} \\
& +(\operatorname{ceil} \gamma-\gamma)\left\{f_{\imath}+\frac{\Delta t}{2}\left[\frac{\chi}{\tau}\left(f_{\imath}^{(0)}-f_{\imath}\right)+\mathcal{F}_{\imath} f_{\imath}^{(0)}\right]\right\}_{\boldsymbol{x}-\frac{\text { floor } \gamma}{\gamma} \Delta t \boldsymbol{c}_{\imath}}^{t-\Delta t}
\end{aligned}
$$

The pressure correction for the BGK approach and the BBGKY approach both require derivatives of the macroscopic quantities. In this research, these derivatives are calculated by second order accurate central differences whenever possible. Near boundaries, where central differences cannot be applied, a onesided second order scheme is used to calculate the derivative. 


\subsection{Boundary conditions}

In this research the flow about a stationary object will be considered in a flow with uniform velocity and temperature at inflow and uniform pressure at outflow. Furthermore, boundary conditions need to be applied at the sides of the domain, were neither velocity, temperature nor pressure are imposed, but reflections should be prevented. First the wall-boundary conditions for the stationary object are presented. No-slip boundary conditions are applied with the walltemperature assumed to be equal to the fluid-temperature at the wall, i.e. the fluid is not heated nor cooled due to temperature differences between fluid and wall. The no-slip boundary conditions result in zero velocity at the wall. Therefore, the chance to observe a molecule moving towards the wall with a specific velocity will be equal to the chance to observe a molecule moving away from the wall at the exact opposite velocity, resulting in

$$
f\left(\boldsymbol{c} ; \boldsymbol{x}_{\mathrm{wall}}, t\right)=f\left(-\boldsymbol{c} ; \boldsymbol{x}_{\mathrm{wall}}, t\right) .
$$

These are the so-called bounce-back boundary conditions. Integration of the Boltzmann equation along its Lagrangian can then be split in two parts,

$$
\int_{t_{i}}^{t+1} \frac{\mathrm{d} f}{\mathrm{~d} t}(\boldsymbol{c} ; \boldsymbol{x}(t), t) \mathrm{d} t=\int_{t_{i}}^{t_{\mathrm{wall}}} \frac{\mathrm{d} f}{\mathrm{~d} t}(-\boldsymbol{c} ; \boldsymbol{x}(t), t) \mathrm{d} t+\int_{t_{\mathrm{wall}}}^{t_{i+1}} \frac{\mathrm{d} f}{\mathrm{~d} t}(\boldsymbol{c} ; \boldsymbol{x}(t), t) \mathrm{d} t
$$

with

$$
t_{\mathrm{wall}}=t_{i+1}+\frac{\boldsymbol{c}}{\|\boldsymbol{c}\|^{2}} \cdot\left(\boldsymbol{x}\left(t_{i+1}\right)-\boldsymbol{x}_{\mathrm{wall}}\right) .
$$

The bounce-back conditions are also exploited for interpolation of the distribution function, required during the calculation of the right-hand side of the discretized lattice Boltzmann equation. Macroscopic quantities required for the right-hand side of the equation are extrapolated linearly in the direction of the considered molecular velocity. Note that the so-called stair-casing effect is avoided, as each solid-wall is treated exactly at its location instead of either half-way in between nodes or at nodes.

For external boundary conditions, an often used approach is to enforce the velocity on the inlet and density on the outlet by redistribution of the distribution function. To take into account temperature effects, the temperature should also in some way be enforced by such a redistribution. For isothermal calculations, enforcing the density results in the desired a pressure boundary condition. However, for thermal calculations, temperature influences the pressure as well, 
so that the density should be enforced as a result of the local temperature and the desired pressure. Moreover, if the other sides of the domain are not solid walls, some sort of free stream boundary conditions should be imposed.

In this research, another approach is considered. At the boundary, the equilibrium distribution function for incoming distributions belong to the enforced boundary conditions.

$$
f_{\imath}^{(0), \mathrm{bc}}\left(\rho_{\mathrm{b} c}, \boldsymbol{u}_{\mathrm{b} c}, T_{\mathrm{b} c}\right) \equiv \frac{\rho_{\mathrm{b} c}}{m}\left(\frac{m}{2 \pi k T_{\mathrm{b} c}}\right)^{\frac{\mathcal{D}}{2}} e^{-\frac{m\left\|c_{\imath}-\boldsymbol{u}_{\mathrm{b} c}\right\|^{2}}{2 k T_{\mathrm{b} c}}},
$$

with

\begin{tabular}{|ccc|}
\hline Inlet & Outlet & Sides \\
Temperature and velocity & Pressure & Free flow \\
$\rho_{\mathrm{b} c}=\rho$ & $\left\{\rho_{\mathrm{b} c}: p\left(\rho_{\mathrm{b} c}, T\right)=P_{\text {outlet }}\right\}$ & $\rho_{\mathrm{b} c}=\rho$ \\
$\boldsymbol{u}_{\mathrm{b} c}=\boldsymbol{U}_{\text {intlet }}$ & $\boldsymbol{u}_{\mathrm{b} c}=\boldsymbol{u}$ & $\boldsymbol{u}_{\mathrm{b} c}=\boldsymbol{u}$ \\
$T_{\mathrm{b} c}=T_{\text {inlet }}$ & $T_{\mathrm{b} c}=T$ & $T_{\mathrm{b} c}=T$ \\
\hline
\end{tabular}

Furthermore, it is assumed that the distribution function outside the domain $f^{\mathrm{bc}}$ is equal to its local equilibrium distribution and, as a result, satisfies the equation

$$
\frac{\mathrm{d} f^{\mathrm{b} c}}{\mathrm{~d} t}=\mathcal{F} f^{(0), \mathrm{bc}} .
$$

The analytic solution to this equation provides the distribution function outside the domain. In the applied Crank-Nicholson scheme, the resulting equation at the boundary becomes

$$
\begin{gathered}
\left\{f_{\imath}-\frac{\Delta t}{2}\left[\frac{\chi}{\tau}\left(f_{\imath}^{(0), \mathrm{bc}}-f_{\imath}\right)+\mathcal{F}_{\imath} f_{\imath}^{(0), \mathrm{bc}}\right]\right\}_{\boldsymbol{x}_{\mathrm{bc}}}^{t} \\
=\left\{f_{\imath}^{(0), \mathrm{bc}} e^{-\mathcal{F}_{\imath} \Delta t}+\frac{\Delta t}{2} \mathcal{F}_{\imath} f_{\imath}^{(0), \mathrm{bc}} e^{-\mathcal{F}_{\imath} \Delta t}\right\}_{\boldsymbol{x}_{\mathrm{bc}}}^{t} .
\end{gathered}
$$

Note that the distribution only depends on variables at the new time-step. However, the coupling of the macroscopic quantities to the solution of the other distribution functions introduces the time-dependency. The solution at the boundary is not necessarily at local equilibrium and macroscopic quantities are allowed to differ slightly from the boundary conditions. However, for the time-step tending to zero, the incoming distribution functions will be equal to the equilibrium distribution of the boundary conditions. 


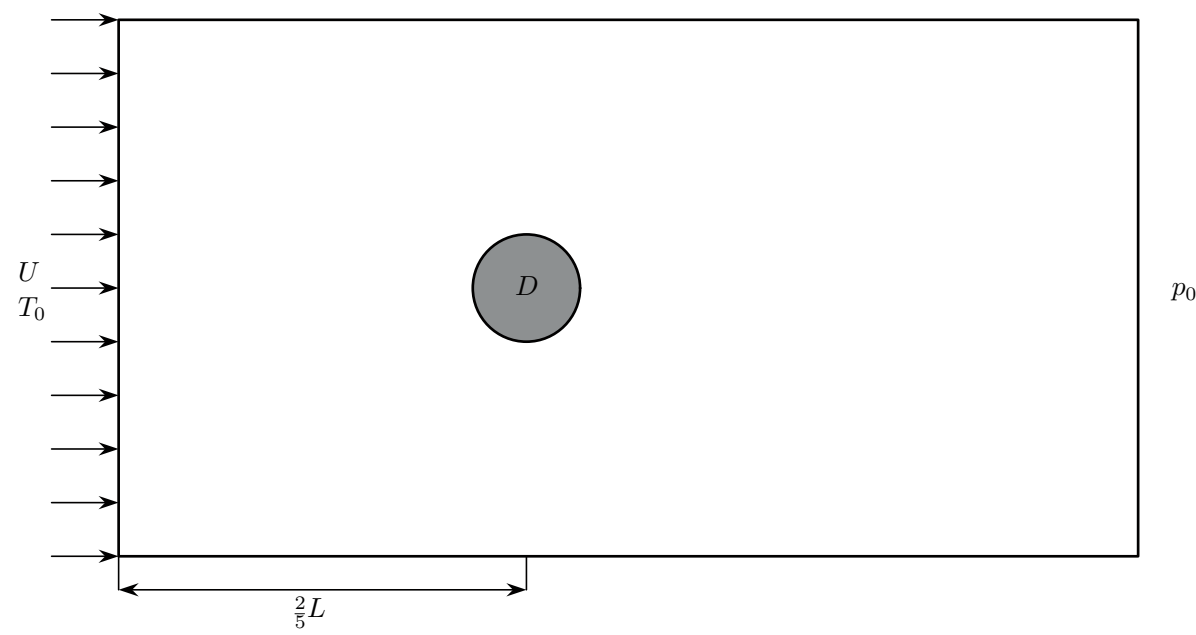

Figure 8.3: The simulation set-up for the flow around a sphere moving in the negative $z$-direction at constant velocity $U$ relative to the flow field. Velocity and temperature are imposed at inlet, while pressure is imposed at the outlet.

$$
\begin{array}{cccc}
T_{0}[\mathrm{~K}] & p_{0}\left[\mathrm{~N} \mathrm{~m}^{-2}\right] & \operatorname{Re}[-] & D[\mathrm{~m}] \\
298.15 & 1 \times 10^{5} & 250 & 7.0625 \times 10^{-3}
\end{array}
$$

Table 8.2: Parameters used in the simulation.

\subsection{Simulation set-up}

The impulsively started motion of water about a sphere is considered, as impulsively started motion will be a first step to study history effects and mimic the experiments of Mordant \& Pinton [32] in a simulation. The domain is presented in figure 8.3. The parameters used in the numerical simulation are presented in table 8.2. The lattice-distance $\Delta x$ is a function of diameter of the sphere and the number of points used to describe the domain. The lattice-distance, the width $W$, the length $L$ of the domain and the position of the sphere become

$$
\Delta x=\frac{8}{n_{y}+8} D
$$




$$
\begin{gathered}
W=\frac{8 n_{y}}{n_{y}+8} D, \\
L=\frac{8 n_{z}}{n_{y}+8} D, \\
\boldsymbol{x}_{\text {Sphere }}=\left(\begin{array}{c}
\frac{W}{2} \\
\frac{W}{2} \\
\frac{2}{5} L
\end{array}\right),
\end{gathered}
$$

where $n_{y}$ and $n_{z}$ are the number of points in $y$-direction and $z$-direction, respectively. $\Delta x$ has been related to the number of points in $y$-direction such that a balance is found between the resolution about the sphere and the distance between the sphere and the boundaries for very low number of points in $y$-direction. The number of points in $x$-direction is taken equal to the number of points in $y$-direction.

\subsection{Results and discussion}

Simulations have been performed for five test cases

- An isothermal ideal gas at $T=T_{0}$, with the density of water;

- An ideal gas initially at $T=T_{0}$, for which the lattice speed and relaxation time are adapted such that $3 \overline{C_{0}^{2}} \approx 1498^{2}\left[\mathrm{~m}^{2} \mathrm{~s}^{-2}\right]$ in order to mimic the speed of sound of water at $T_{0}=298.15[\mathrm{~K}]$, which is $1498\left[\mathrm{~m} \mathrm{~s}^{-1}\right]$.

- Isothermal water at $T=T_{0}$ using the BGK approach with pressure correction;

- (Thermal) Water initially at $T=T_{0}$ using the BGK approach with pressure correction;

- Isothermal water at $T=T_{0}$ using the BBGKY approach;

Temperature dependent simulations for water using the BBGKY approach have not been performed in present research.

Figure 8.4 shows the force on the sphere during an impulsively started flow for each approach, using the same grid and time-step. A higher lattice speed for the ideal-gas approach clearly improves the location and height of the initial peak in the simulations. However, its peak force is still lower and its shape is different. Moreover, following the initial peak, the force remains too high 


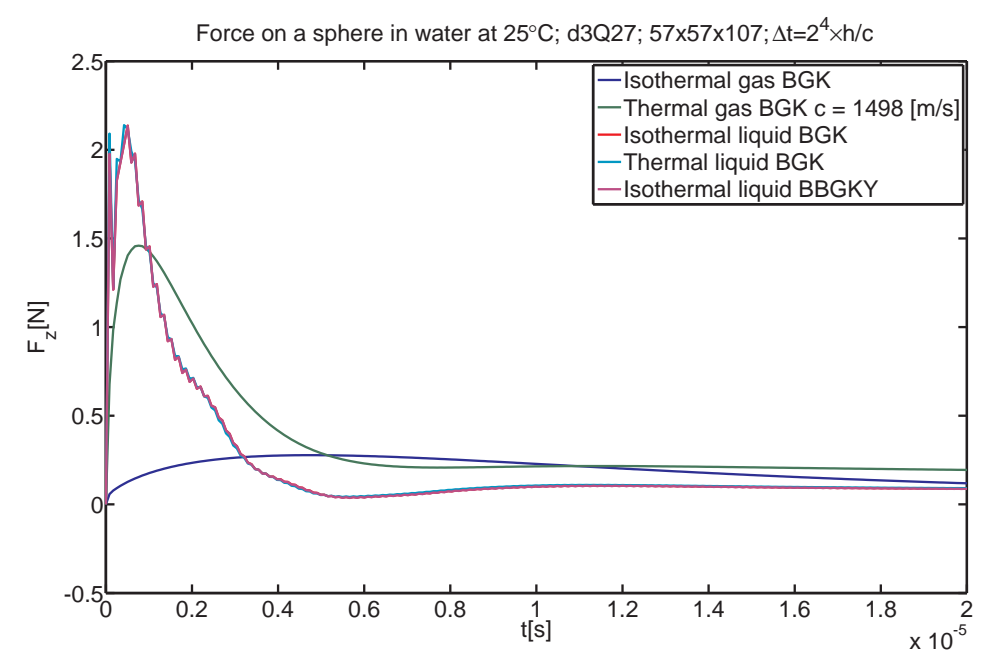

Figure 8.4: Force on a sphere for different LBM models at the conditions reported in table 8.2 for a mesh of $57 \times 57 \times 107$ points.

compared to the force predicted by the pressure corrected BGK and BBGKY simulations. The results for the isothermal water-models are almost equal, although minor differences of the order of $0.1 \%$ can be observed when taking a close look to the data. Although, both models will have a slightly different behaviour due to differences in relaxation time, the results are dominated by the forcing terms due to near incompressibility fo water. Moreover, the forcing term of the isothermal models only differs by a term containing the divergence of the velocity, which should be zero for incompressible flow. Finally, it can be observed that the thermal effects for the BGK approach with pressure correction only show minor differences with respect to the results for the isothermal case.

For all water-models results show oscillatory behaviour. Therefore, the influence of the time-step has been investigated for the isothermal BBGKY model. Figure 8.5 show a comparison of the result with the time-step used for the simulations of figure 8.4 and the result obtained with half the time-step. With half the time-step, the oscillatory behaviour is only visible for the first time-step. However, the smoothed behaviour for the coarser time-step is the same as obtained for the smaller time-step. This implies that dependency on the time-step 


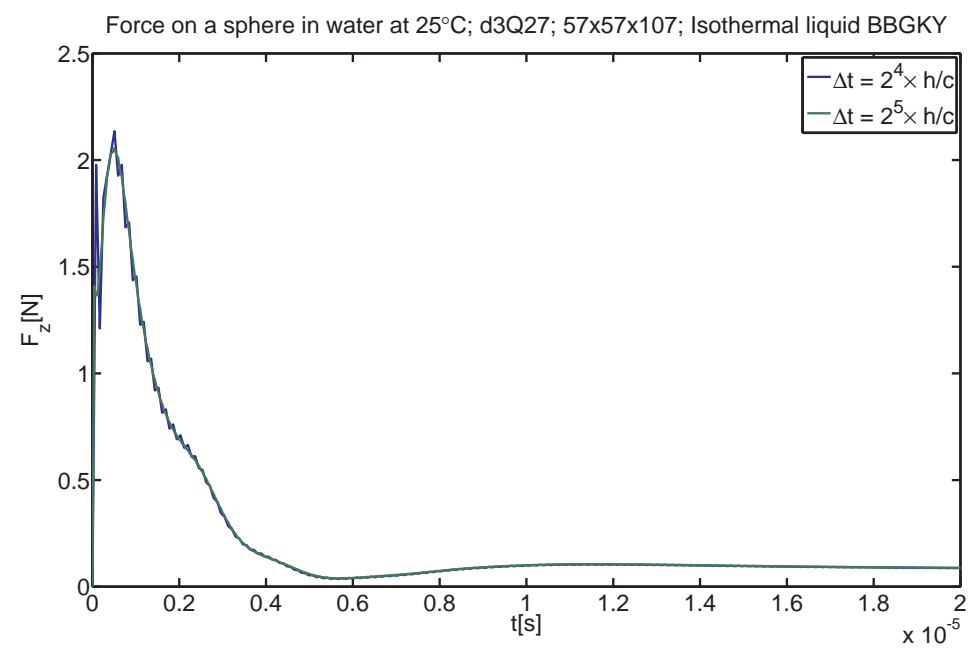

Figure 8.5: Force on a sphere using two different time-steps for the isothermal BBGKY case of figure 8.4.

is not very high.

Finally, the grid-dependency has been investigated. Figure 8.6 shows a comparison of results for different grid resolutions using the smallest time-step as used in the time-step investigation. The differences between the results for these grids are much larger than the differences caused by a different time-step. Therefore, initial improvement of the results should be obtained by increasing the grid-resolution, while retaining the same time-step.

Differences in results are not only visible in the force on the sphere, but also in the flow field. Figure 8.7 shows iso-contours of the magnitude of the velocity after 1442 time-steps. For the case of an ideal gas with an increased speed of sound the maximum velocity is $3.22 \times 10^{-2}\left[\mathrm{~m} \mathrm{~s}^{-1}\right]$. However, for the isothermal BBGKY case the maximum velocity is $3.296 \times 10^{-2}\left[\mathrm{~m} \mathrm{~s}^{-1}\right]$. Moreover, the shape of the iso-countours is different for these two simulations. For the case of an ideal gas, the shape is much more ellipsoidal, while the results for the isothermal BBGKY case shows much higher gradients perpendicular to the flow in the plane in which the sphere is located.

Overall, it can be concluded that an ideal gas model is not capable of simulating a liquid due to the differences in behaviour of pure collision effects for 


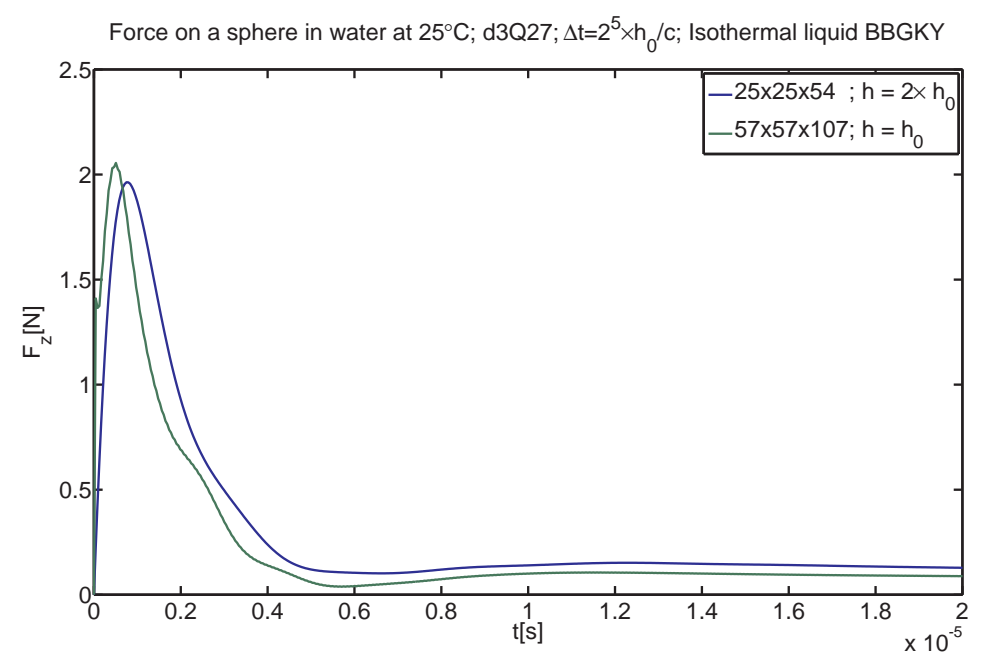

Figure 8.6: Force on a sphere for different grid-spacings for the isothermal BBGKY case with the smallest time-step of figure 8.4.

an ideal gas, and a combination of attractive-repulsive behaviour for a liquid. If liquid is approximated by an ideal gas, care should be taken to consider the implications of neglecting such effects. It has also been shown that using a BGK model with pressure correction does not show much differences with respect to the slightly more complicated BBGKY model. However, more differences might be visible with results from temperature dependent calculations. 

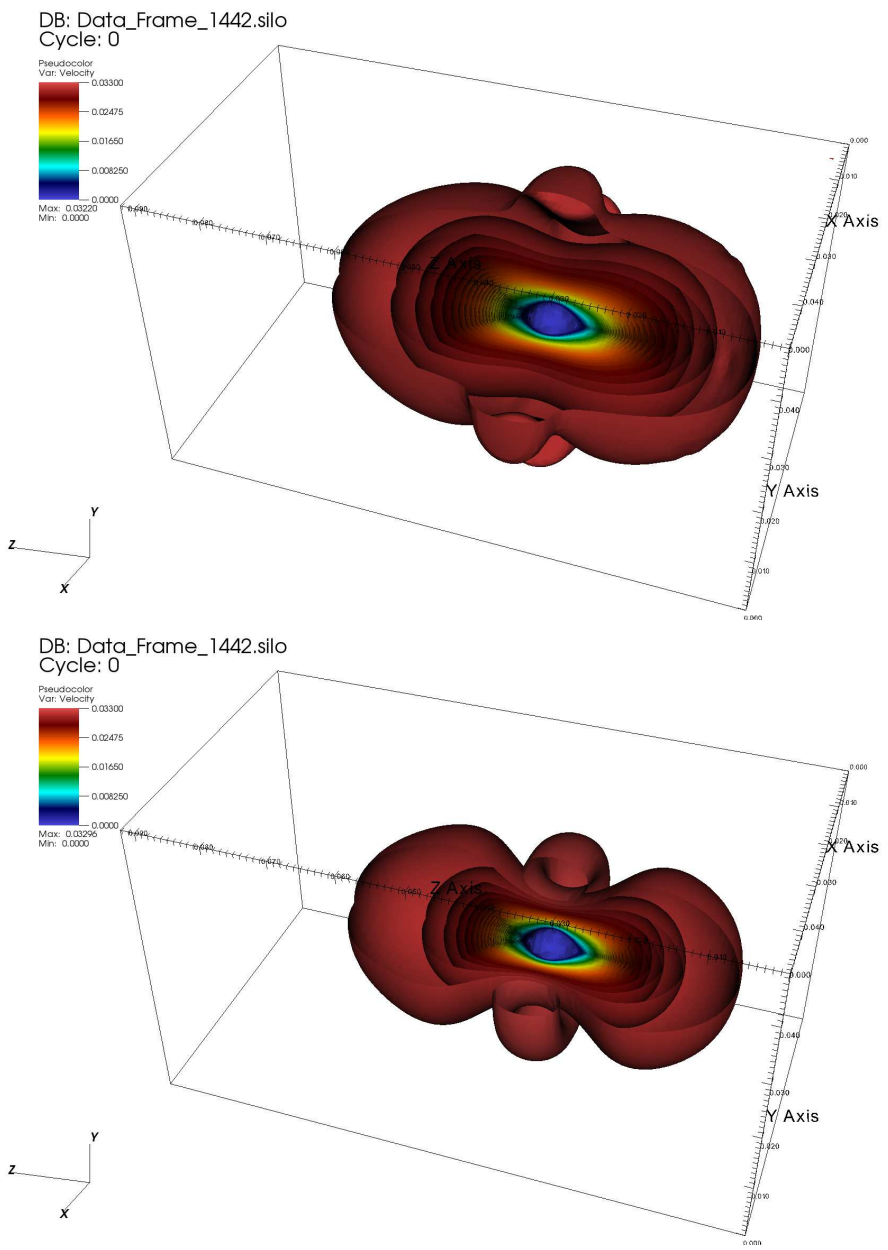

Figure 8.7: Iso-contours of the magnitude of the velocity for ideal-gas with increased speed of sound (upper) and the isothermal BBGKY model (lower). Result after after 1442 time-steps for the conditions reported in table 8.2. The inlet is at the right-hand side of the presented domain. 



\section{CHAPter 9}

\section{CONCLUDING REMARKS AND RECOMMENDATIONS}

\subsection{Faxèn forces, lift forces and torque}

In chapter 2 , the equations of motion for a particle have been derived. In chapter 3 the force on that particle has been approximated by a macroscopic force model. From the various contributions to this force, the influence of the history force has been investigated in detail. The Faxèn force effects have not been considered in the presented case of swirling flow for oil-water separation. However, since the Laplacian of the flow field is not zero, these forces should be considered in further research, as they influence the balance between the history force and the ones due to instantaneous effects.

During the derivation of the macroscopic force model in chapter 3 an extension of the Faxèn force terms is proposed, which takes the effect of higher order derivatives in the macroscopic variables and derived coefficients into account into account. For cases for which the original Faxèn force is important, adapting the involved coefficients will be important. This should be based on a combination of experiments and fully resolved simulations.

During the derivation of the lift forces an expression for the lift coefficient was proposed for the Saffman lift, adapting the expression proposed by Mei (1992) [29] resulting in a continuous expression. For the present case, effects of lift-forces turned out to be negligible and no attention was given to the proposed expression. However, the continuous nature of the proposed expression will be 
useful in numerical schemes, especially in regions where other expressions are discontinuous. The expression should first be validated quantitatively in a more thorough comparison to experimental data.

For cases for which lift cannot be neglected, the torque on a particle becomes important. Such a case would be ideal to investigate whether it is sufficient to account for the contributions in the torque using Faxèn force-like torques caused by drag, stress-divergence, added mass and lift. This would remove the need for a separate expression for the torque.

\subsection{The history force and its influence}

In chapter 4, the equations of motions have been discretized. Discretization of the history integral has been described in detail for a Reynolds number dependent history force using partial integration and a change of integration variables. For higher order time-integration methods for the differential equation, the proposed scheme should be extended to obtain a higher order accurate expression for the integration of the history force as well. For this, higher order terms of the derivative with respect to the history time in the history force integral are required.

In section 3.8 an improved kernel for the history force has been proposed. In chapter 5 , this kernel has been validated using existing experimental data of settling spheres. However, the proposed expression is still not able to correct the drag force such that a good match is obtained for some of the experimental data considered.

One major problem for validating the history force, is that it cannot be directly measured. The experimentally obtained history force is a result of the measured particle accelerations and measured particle mass as well as the drag that is computed with the chosen drag coefficient based on its measured particle velocity and particle diameter. This implies that each drag coefficient corresponds to a specific fit for the history force. Therefore, it is important to first determine the best drag coefficient, at least in the region of interest, before fitting the history force. Moreover, a lack of experimental data in the range of very low Reynolds numbers makes it difficult to validate the various drag-correlations and needs attention in order to obtain a better expression for the history force. Therefore, an experimental study or numerical study based on fully resolved simulations should be carried out in order to obtain better prediction of the drag and history force.

In chapter 6 , the history force has been applied to the case of swirling flow for 
oil-water separation. It has been shown that the influence of the history force is of similar order of magnitude as the influence of Reynolds number effects on the drag. Therefore, the history force cannot be neglected for the case of oil-droplets in a swirling flow. However, it has also been shown that for the considered case the history force could be approximated quite well with a draglike expression. Therefore, in order to improve predictions for such flows, it should be investigated whether this expression holds for all droplets of relevant diameter released at various radii in the swirling flow separator.

The results of the simulations including the history force suggest that history force effects should be included when predicting the required length of the separator based on particle trajectories and based on its droplet size dependent cut-off ratio. Including the effects of the history force will result in longer residence times for droplets and therefore, negatively affect the accuracy of the separation performance if not considered.

Whether or not it is necessary to take the history force into account not only depends on the case considered, but also on the required output data. Therefore, a study to investigate the importance of the history force in a particle motion problem should be performed for at least one case for a representative particle. If results show that the history force is important, the nature of the history force in that specific case can be investigated in order to determine possibilities to approximate the history force by taking into account only part of the integral with a cut-off window or by approximating the history force using instantaneous flow information.

\subsection{Efficient collision detection}

In chapter 7 an efficient collision detection scheme has been developed. The scheme decreases calculation time significantly, in particular for cases for which the bounding volume of particle presence is large with respect to the size of the particles. This enables an efficient calculation in which potential collision between particles are not only detected, but proper particle interaction models can be applied. Moreover, soft collision models in which long range particle interactions are included can make use of the infrastructure of the present implementation in order to carry multi-grid summations. Such soft collision models will be of importance when it is appropriate to account for the effects of flow modification by the motion of the particles. Other aspects, such as particle distribution models will affect the efficiency of the scheme. Therefore a first step is to investigate the efficiency of the model for various particle size distributions, 
such as a log-normal distribution.

\subsection{A lattice Boltzmann method for water}

In chapter 8, two models for a Lattice Boltzmann Method for the flow of water have been introduced. One model uses the standard BGK approximation with a pressure gradient correction term. The other model uses a BBGKY approximation to treat water as a dense-gas. It has been shown that both models lead to comparable results for the flow of water about a sphere, while an ideal-gas approach for the flow of water does not capture the nature of nearincompressibility of a liquid. The results for an ideal gas show flow behaviour that is visibly different from the flow behaviour predicted utilizing the water models. Moreover, an integrated time-dependent effect, such as the force on a particle, is not captured accurately. Therefore, using an ideal-gas approach for simulations of a liquid should be assessed thoroughly.

The proposed method itself is not yet validated with experimental data, due to the required computational effort. As calculations are computationally expensive, the first step that should be taken is to simplify the problem and compare results to experimental data or to accurate numerical data. One way for such a problem simplification is to reduce the problem from 3-D to 2-D and compare the results to experimental and numerical data for the 2-D flow about a cylinder.

Also, validation should be separated in two parts. One part is the validation of the proposed quadrature scheme, and the other part is the validation of the proposed model for the liquid. The quadrature scheme should be validated for a gas for near-isothermal flows as well as for temperature dependent cases, while the proposed liquid model can be validated following the validation of the quadrature scheme.

Furthermore, the proposed methods lack a model for the internal energy of the molecules. This will be another line of investigation, especially if the model is to be applied to temperature dependent problems such as RayleighBénard convection. Also, for such temperature dependent problems, the balance between relaxation times for effects due to heat conduction and those for viscous effects becomes important as well.

In order to be able to reproduce results of experiments for settling spheres, various aspects of the implementation should be improved. First, local grid refinement should help rendering the simulation sufficiently accurate in the region of interest, while having a large enough domain to reduce effects due to the 
boundaries of the computational domain. Due to the decoupling of space and time, the proposed method is less restrictive with regard to local grid refinement.

Second, presently the case considered is that of a sphere fixed in an impulsively started continuous flow. In order to reproduce the experiments, either a scheme for a moving grid, or a scheme for a moving sphere should be implemented. Again the nature of the proposed Boltzmann method, which uses interpolated values for the variables and forcing terms at the previous time-step would make a moving grid scheme feasible. The treatment of the boundary conditions will need to be considered with care, specifically with respect to the location and to the influence of the boundary on the distribution function.

Also, the solid wall boundary conditions need investigation for various problems. A scheme for a moving boundary would be implemented by mirroring about the velocity of the boundary instead of mirroring about zero. The proposed scheme already applies a scheme to approximate the deviation of a distribution function by a known distribution function with a small change in molecular velocity and the integral of the equilibrium distribution function in between the actually required molecular velocity and the velocity of the known distribution. This scheme will makes it possible to apply such mirroring about an arbitrary velocity of the boundary. Temperature dependent boundary conditions also need investigation, as mirroring is not capable of capturing heat transfer. Again the proposed method could be exploited by introducing such temperature effects to obtain the jump in the distributions at the boundary.

Finally, it should be observed that the BBGKY approximation makes a clear distinction between short-range and long-range effects. Preferably, the equation of state, used to describe the relation between pressure, density and temperature should also have this distinction between short-range and long-range effects. If this distinction is not clear in the equation of state, it is recommended to use the pressure corrected BGK instead. 



\section{BIBLIOGRAPHY}

[1] A. A. Al-Shamrani, A. James \& H. Xiao (2002). Destabilisation of oilwater emulsions and separation by dissolved air flotation. Water Research, 36(6) 1503-1512. ISSN 0043-1354. doi:10.1016/S0043-1354(01)00347-5.

[2] A. A. Al-Shamrani, A. James \& H. Xiao (2002). Separation of oil from water by dissolved air flotation. Colloids and Surfaces A: Physicochemical and Engineering Aspects, 209(1) 15-26. ISSN 0927-7757. doi:10.1016/ S0927-7757(02)00208-X.

[3] ANSYS, Inc., Southpointe, 275 Technology Drive, Canonsburg, PA 15317 (2006). ANSYS CFX-Solver Theory guide.

[4] T. R. Auton, J. C. R. Hunt \& M. Prud'Homme (1988). The force exerted on a body in inviscid unsteady non-uniform rotational flow. Journal of Fluid Mechanics, 197 241-257.

[5] Fabian A. Bombardelli, Andrea E. Gonzalez \& Yarko I. Nino (2008). Computation of the particle Basset force with a fractional-derivative approach. Journal of Hydraulic Engineering, 134(10) 1513-1520.

[6] Phillip P. Brown \& Desmond F. Lawler (2003). Sphere drag and settling velocity revisited. Journal of Environmental Engineering, 129(3) 222-231. doi:10.1061/(ASCE)0733-9372(2003)129:3(222).

[7] E. Carissimi, Jan D. Miller \& Jorge Rubio (2007). Characterization of the high kinetic energy dissipation of the Flocs Generator Reactor (FGR). International Journal of Mineral Processing, 85(1-3) 41-49. ISSN 03017516. doi:10.1016/j.minpro.2007.08.001. 
[8] Sydney Chapman \& T. G. Cowling (1991). The mathematical theory of non-uniform gases. Cambridge Mathematical Library. Cambridge University Press, The Pitt Building, Trumpington Street, Cambridge CB2 1RP, 3 edition. ISBN 9780521408448.

[9] Shiyi Chen \& Gary D. Doolen (1998). Lattice Boltzmann method for fluid flows. Annual Review of Fluid Mechanics, 30 329-364. ISSN 00664189 .

[10] Nian-Sheng Cheng (2009). Comparison of formulas for drag coefficient and settling velocity of spherical particles. Powder Technology, 189(3) 395-398. ISSN 0032-5910. doi:10.1016/j.powtec.2008.07.006.

[11] Miroslav Colic, Dwain E. Morse, Wade O. Morse \& Jan D. Miller (2005). New developments in mixing, flocculation and flotation for industrial wastewater pretreatment and municipal wastewater treatment. Proceedings of the Water Environment Federation, 2005(14) 2380-2407.

[12] Miroslav Colic, Wade O. Morse \& Jan D. Miller (2007). The development and application of centrifugal flotation systems in wastewater treatment. International Journal of Environment and Pollution, 30(2) 296-312. ISSN 0957-4352. doi:10.1504/IJEP.2007.014706.

[13] J. Crank \& P. Nicolson (1947). A practical method for numerical evaluation of solutions of partial differential equations of the heat-conduction type. Mathematical Proceedings of the Cambridge Philosophical Society, 43 50-67. ISSN 1019-7168. doi:10.1007/S0305004100023197.

[14] J. CRAnk \& P. Nicolson (1996). A practical method for numerical evaluation of solutions of partial differential equations of the heat-conduction type. Advances in Computational Mathematics, 6 207-226. ISSN 10197168. doi:10.1007/BF02127704. Reprint from [13].

[15] Clayton T. Crowe, Martin Sommerfeld \& Yutaka Tsuji (1998). Multiphase flows with droplets and particles. CRC Press, 6000 Broken Sound Parkway, NW, (Suite 300) Boca Raton, Florida 33487, USA.

[16] A. J. Dorgan \& E. Loth (2007). Efficient calculation of the history force at finite Reynolds numbers. International Journal of Multiphase Flow, 33(8) 833-848. ISSN 0301-9322. doi:10.1016/j.ijmultiphaseflow.2007.02. 005. 
[17] Dirk F. van Eijkeren \& Harry W. M. HoeiJmakers (2010). Influence of the history term in a Lagrangian method for oil-water separation. In 7th International Conference on Multiphase Flow - ICMF 2010 Proceedings, page 6.2.1. International Conference on Multiphase Flow (ICMF).

[18] Dirk F. van Eijkeren \& Harry W. M. Hoeijmakers (2012). History force and drag correlation in a Lagrangian method applied to oil-water separation. In Proceedings of the 8th international conference on CFD in the oil $\&$ gas, metallurgical and process industries, edited by Jan Erik Øvrebø Olsen \& Stein Tore Johansen, pages 111:1-111:10. SINTEF. ISBN 9788214052626. CFD2011, Trondheim, Norway, June 2011.

[19] Dirk F. van Eijkeren \& Harry W. M. Hoeijmakers (2012). History force and inertia effects applied to swirling flow produced water treatment. In The 9th international conference on CFD in the minerals and process industries, edited by C. B. Solnordal, P. Liovic \& P. J. Witt, pages 044:1-044:6. CSIRO. ISBN 9781922173010. CFD2012, Melbourne, Australia, 10-12 December 2012.

[20] Dirk F. van EiJkeren \& Harry W. M. Hoeijmakers (2013). the influence of the history force in Lagrangian particle tracking applied to swirling flow produced water treatment. In International Conference on Multiphase Flow 2013 Jeju, Korea, page 644. ICMF. ICMF2013, Jeju, Korea, 26-31 May 2013.

[21] Dirk F. van Eijkeren, Harry W. M. Hoeijmakers \& Thomas Krebs (2015). Efficient collision detection in a simulated hydrocyclone. Journal of Dispersion Science and Technology, 36(10) 1483-1492. ISSN 0193-2691.

[22] L. O. Filippov, R. Joussemet \& R. Houot (2000). Bubble spargers in column flotation: Adaptation to precipitate flotation. Minerals Engineering, 13(1) 37-51. ISSN 0892-6875. doi:10.1016/S0892-6875(99)00148-X.

[23] M. J. Francis \& R. M. Pashley (2006). The effect of de-gassing on the dispersion of fine oil droplets in water. Colloids and Surfaces A: Physicochemical and Engineering Aspects, 287(1-3) 36-43. ISSN 0927-7757. doi: 10.1016/j.colsurfa.2006.03.026.

[24] Xiaoyi He \& Gary D. Doolen (2002). Thermodynamic foundations of kinetic theory and lattice Boltzmann models for multiphase flows. Journal of Statistical Physics, 107 309-328. ISSN 0022-4715. doi:10.1023/A: 1014527108336. 
[25] Jesús A. Izaguirre, Scott S. Hampton \& Thierry Matthey (2005). Parallel multigrid summation for the $N$-body problem. Journal of Parallel and Distributed Computing, 65(8) 949-962. ISSN 0743-7315. doi: 10.1016/j.jpdc.2005.03.006.

[26] C. A. Jeffery \& P. H. Austin (1999). A new analytic equation of state for liquid water. Journal of Chemical Physics, 110(1) 484-496. ISSN 0021-9606.

[27] Christopher J. Lawrence \& Renwei Mei (1995). Long-time behaviour of the drag on a body in impulsive motion. Journal of Fluid Mechanics, 283 $307-327$.

[28] E. Loth \& A. J. Dorgan (2009). An equation of motion for particles of finite Reynolds number and size. Environmental Fluid Mechanics, 9(2) 187-206.

[29] Renwei MeI (1992). An approximate expression for the shear lift force on a spherical particle at finite Reynolds number. International Journal of Multiphase Flow, 18(1) 145-147.

[30] Renwei Mei \& Ronald J. Adrian (1992). Flow past a sphere with an oscillation in the free-stream velocity and unstready drag at finite Reynolds number. Journal of Fluid Mechanics, 237 323-341.

[31] Renwei Mei, Christopher J. Lawrence \& Ronald J. Adrian (1991). Unsteady drag on a sphere at finite Reynolds-number with small fluctuations in the free-stream velocity. Journal of Fluid Mechanics, 233 613-631.

[32] Nicolas Mordant \& Jean-François Pinton (2000). Velocity measurement of a settling sphere. European Physical Journal B, 18(2) 343-352.

[33] Viktor Georgijevch Ponomariov (2009). Study and application of flotation in schemes for waste water treatment. Environmental Reseach, Engineering and Management, 50(4) 51-56. Viktoras Georgijevičius Ponomariovas.

[34] S. I. Rubinow \& Joseph B. Keller (1961). The transverse force on a spinning sphere moving in a viscous fluid. Journal of Fluid Mechanics, 11(03) 447-459. 
[35] L. Schiller \& A. Naumann (1933). Über die grundlegenden berechungen bei der schwerkraftaufbereitung. Vereines Deutscher Ingenieure, 77 318320 .

[36] Renato Silva, Aline Nogueira Da Silveira \& Jorge Rubio (2009). Treatment of acid mine drainage and water reuse: Flotation with microbubbles versus flocs lamellar seetling. In Procemin 2009, edited by Peter Amelunxen, Willy Kracht \& Romke Kuyvenhoven, pages 513-522. fcfm, GECAMIN, 197 Paseo Bulnes, 6th Floor, Santiago, Chile. Proceedings of the VI International Mineral Processing Seminar.

[37] Jesse J. Slot, Laurens J. A. M. Campen, Harry W. M. Hoeijmakers \& Rob F. Mudde (2012). Inline oil-water separation in swirling flow. In Proceedings of the 8th international conference on CFD in the oil 8 gas, metallurgical and process industries, Trondheim, 2011, pages 121:110. ISBN 9788214052626.

[38] Y. S. Touloukian, S. C. Saxena \& P. Hestermans (1975). Viscosity, volume 11 of Thermophysical properties of matter. IFI/Plenum, New YorkWashington. ISBN 0-306-67031-3. 



\section{APPENDiX A}

\section{DERIVATIONS RELATED TO THE EQUATIONS OF MOTION}

In section 2.1 the velocity, position and time rate of rotation of a particle are defined and the equations of motion are presented. Furthermore, derivation of the equation governing the behaviour of particle velocity in time is presented in section 2.1. Derivation of the equations governing the behaviour in time of particle position as well as of the time rate of rotation will be presented in this chapter.

\section{A.1 Particle postion}

The particle position, defined as the centre of mass in equation (2.9), is

$$
\boldsymbol{x}_{\mathrm{p}} \equiv \frac{1}{m} \iiint_{V_{\mathrm{p}}} \rho \boldsymbol{x} \mathrm{d} V,
$$

with the mass $m$ of the particle, defined in equation (2.2) as

$$
m \equiv \iiint_{V_{\mathrm{p}}} \rho \mathrm{d} V .
$$


The change of mass of the particle is equal to the flux of mass $\phi$ through the surface integrated over the surface of the volume

$$
\frac{\mathrm{d} m}{\mathrm{~d} t}=\iint_{\partial V_{\mathrm{p}}} \phi \mathrm{d} S,
$$

with the mass flux, defined in equation (2.5), as

$$
\phi \equiv \widetilde{\rho}\left(\boldsymbol{u}_{\partial V_{\mathrm{p}}}-\boldsymbol{u}\right) \cdot \boldsymbol{n},
$$

with external unit normal vector $\boldsymbol{n}$.

The density $\widetilde{\rho}$, depends on the relative velocity of the boundary with respect to the local velocity, defined in equation (2.4), is

$$
\widetilde{\rho} \equiv \begin{cases}\rho_{\text {added }} & \text { if }\left(\boldsymbol{u}_{\partial V_{\mathrm{p}}}-\boldsymbol{u}\right) \cdot \boldsymbol{n}>0 \\ \rho_{\partial V_{\mathrm{p}}} & \text { if }\left(\boldsymbol{u}_{\partial V_{\mathrm{p}}}-\boldsymbol{u}\right) \cdot \boldsymbol{n}=0 \\ \rho_{\text {removed }} & \text { if }\left(\boldsymbol{u}_{\partial V_{\mathrm{p}}}-\boldsymbol{u}\right) \cdot \boldsymbol{n}<0\end{cases}
$$

The definition of the particle position and the time rate of the change of mass are now used for the change of the particle position in time. The time rate of change of the particle position is

$$
\frac{\mathrm{d} \boldsymbol{x}_{\mathrm{p}}}{\mathrm{d} t}=\frac{\mathrm{d}}{\mathrm{d} t}\left[\frac{1}{m} \iiint_{V_{\mathrm{p}}} \rho \boldsymbol{x} \mathrm{d} V\right] .
$$

Manipulation of the derivative leads to

$$
\frac{\mathrm{d} \boldsymbol{x}_{\mathrm{p}}}{\mathrm{d} t}=\frac{1}{m} \frac{\mathrm{d}}{\mathrm{d} t} \iint_{V_{\mathrm{p}}} \rho \boldsymbol{x} \mathrm{d} V-\frac{\boldsymbol{x}_{\mathrm{p}}}{m} \frac{\mathrm{d} m}{\mathrm{~d} t} .
$$

Applying Leibniz rule to the first integral, and substituting the integral of the flux for the change of mass, leads to

$$
\frac{\mathrm{d} \boldsymbol{x}_{\mathrm{p}}}{\mathrm{d} t}=\frac{1}{m}\left[\iiint_{V_{\mathrm{p}}} \boldsymbol{x} \frac{\partial \rho}{\partial t} \mathrm{~d} V+\iint_{\partial V_{\mathrm{p}}} \tilde{\rho} \boldsymbol{x} \boldsymbol{u}_{\partial V_{\mathrm{p}}} \cdot \boldsymbol{n} \mathrm{d} S\right]-\frac{\boldsymbol{x}_{\mathrm{p}}}{m} \iint_{\partial V_{\mathrm{p}}} \phi \mathrm{d} S .
$$

However, local quantities of the density and velocity inside the particle might not be easily obtained. Moreover, the velocity $\boldsymbol{u}_{\text {partial }_{\mathrm{p}}}$ of the interface, as 
well as the velocity $\boldsymbol{u}$ at the interface are also not easy to obtain. However, a total flux of mass through the surface is often known or prescribed. To be able to evaluate the integrals first the volume integral will be rewritten.

Due to mass conservation, the time derivative of the density becomes minus the divergence of momentum.

$$
\frac{\mathrm{d} \boldsymbol{x}_{\mathrm{p}}}{\mathrm{d} t}=\frac{1}{m}\left[-\iiint_{V_{\mathrm{p}}} \boldsymbol{x} \boldsymbol{\nabla} \cdot(\rho \boldsymbol{u}) \mathrm{d} V+\iint_{\partial V_{\mathrm{p}}} \tilde{\rho} \boldsymbol{x} \boldsymbol{u}_{\partial V_{\mathrm{p}}} \cdot \boldsymbol{n} \mathrm{d} S\right]-\frac{\boldsymbol{x}_{\mathrm{p}}}{m} \iint_{\partial V_{\mathrm{p}}} \phi \mathrm{d} S .
$$

Subsequently, manipulation of the derivative leads to

$$
\begin{aligned}
\frac{\mathrm{d} \boldsymbol{x}_{\mathrm{p}}}{\mathrm{d} t}=\frac{1}{m}\left[\iiint_{V_{\mathrm{p}}} \rho \boldsymbol{u} \cdot \nabla \boldsymbol{x} \mathrm{d} V-\right. & \iiint_{V_{\mathrm{p}}} \boldsymbol{\nabla} \cdot(\rho \boldsymbol{u} \boldsymbol{x}) \mathrm{d} V \\
& \left.+\iint_{\partial V_{\mathrm{p}}} \rho \boldsymbol{x} \boldsymbol{u}_{\partial V_{\mathrm{p}}} \cdot \boldsymbol{n} \mathrm{d} S\right]-\frac{\boldsymbol{x}_{\mathrm{p}}}{m} \iint_{\partial V_{\mathrm{p}}} \phi \mathrm{d} S .
\end{aligned}
$$

The gradient of the position results in the unit matrix. Moreover, the second integral can be transformed to a surface integral using the divergence theorem. Therefore the equation becomes

$$
\begin{array}{r}
\frac{\mathrm{d} \boldsymbol{x}_{\mathrm{p}}}{\mathrm{d} t}=\frac{1}{m}\left[\iiint_{V_{\mathrm{p}}} \rho \boldsymbol{u} \mathrm{d} V-\iint_{\partial V_{\mathrm{p}}} \widetilde{\rho} \boldsymbol{x} \boldsymbol{u} \cdot \boldsymbol{n} \mathrm{d} S+\iint_{\partial V_{\mathrm{p}}} \widetilde{\rho} \boldsymbol{x} \boldsymbol{u}_{\partial V_{\mathrm{p}}} \cdot \boldsymbol{n} \mathrm{d} S\right] \\
-\frac{\boldsymbol{x}_{\mathrm{p}}}{m} \iint_{\partial V_{\mathrm{p}}} \phi \mathrm{d} S .
\end{array}
$$

Finally the differential equation for particle position becomes

$$
\frac{\mathrm{d} \boldsymbol{x}_{\mathrm{p}}}{\mathrm{d} t}=\boldsymbol{v}+\frac{1}{m} \iint_{\partial V_{\mathrm{p}}}\left(\boldsymbol{x}-\boldsymbol{x}_{\mathrm{p}}\right) \phi \mathrm{d} S .
$$


128 Appendix A. Derivations related to the Equations of motion

\section{A.2 Particle rate of rotation}

The equation for conservation of angular momentum with respect to a fixed frame of reference, provided in equation (2.15), is

$$
\begin{aligned}
\frac{\mathrm{d}}{\mathrm{d} t} \iiint_{V_{\mathrm{p}}} \boldsymbol{x} \times \rho \boldsymbol{u} \mathrm{d} V=\iint_{\partial V_{\mathrm{p}}} \boldsymbol{x} \times(\boldsymbol{\sigma} \cdot \boldsymbol{n}) \mathrm{d} S+\iiint_{V_{\mathrm{p}}} \rho \boldsymbol{x} & \times \boldsymbol{f} \mathrm{d} V \\
& +\iint_{\partial V_{\mathrm{p}}} \phi \boldsymbol{x} \times \boldsymbol{u} \mathrm{d} S .
\end{aligned}
$$

To obtain an equation for conservation of angular momentum with respect to the particle position, the location with respect to the particle position is introduced, defined equation (2.19), as

$$
\chi \equiv \boldsymbol{x}-\boldsymbol{x}_{\mathrm{p}}
$$

The equation for conservation of angular momentum becomes

$$
\begin{aligned}
& \frac{\mathrm{d}}{\mathrm{d} t} \iint_{V_{\mathrm{p}}} \boldsymbol{\chi} \times \rho \boldsymbol{u} \mathrm{d} V=\iint_{\partial V_{\mathrm{p}}} \boldsymbol{\chi} \times(\boldsymbol{\sigma} \cdot \boldsymbol{n}) \mathrm{d} S+\iiint_{V_{\mathrm{p}}} \rho \boldsymbol{\chi} \times \boldsymbol{f} \mathrm{d} V \\
&+\iint_{\partial V_{\mathrm{p}}} \phi \boldsymbol{\chi} \times \boldsymbol{u} \mathrm{d} S-\boldsymbol{x}_{\mathrm{p}} \times\left\{\frac{\mathrm{d}}{\mathrm{d} t} \iiint_{V_{\mathrm{p}}} \rho \boldsymbol{u} \mathrm{d} V-\iint_{\partial V_{\mathrm{p}}} \boldsymbol{\sigma} \cdot \boldsymbol{n} \mathrm{d} S\right. \\
&\left.-\iiint_{V_{\mathrm{p}}} \rho \boldsymbol{f} \mathrm{d} V-\iint_{\partial V_{\mathrm{p}}} \phi \boldsymbol{u} \mathrm{d} S\right\}-\frac{\mathrm{d} \boldsymbol{x}_{\mathrm{p}}}{\mathrm{d} t} \times \iiint_{V_{\mathrm{p}}} \rho \boldsymbol{u} \mathrm{d} V .
\end{aligned}
$$

In the equation, the equation for conservation of momentum can be recognized, while the time derivative of the particle location is equal to the particle velocity and an integrated mass flux effect. Also the last integral is the average particle momentum, leading to

$$
\begin{aligned}
& \frac{\mathrm{d}}{\mathrm{d} t} \iiint_{V_{\mathrm{p}}} \boldsymbol{\chi} \times \rho \boldsymbol{u} \mathrm{d} V=\iint_{\partial V_{\mathrm{p}}} \boldsymbol{\chi} \times(\boldsymbol{\sigma} \cdot \boldsymbol{n}) \mathrm{d} S+\iiint_{V_{\mathrm{p}}} \rho \boldsymbol{\chi} \times \boldsymbol{f} \mathrm{d} V \\
& \quad+\iint_{\partial V_{\mathrm{p}}} \phi \boldsymbol{\chi} \times \boldsymbol{u} \mathrm{d} S-\boldsymbol{x}_{\mathrm{p}} \times\left\{\frac{\mathrm{d}}{\mathrm{d} t}(m \boldsymbol{v})-\boldsymbol{F}_{\mathrm{p}}-\boldsymbol{\Psi}\right\}-(\boldsymbol{v}+\boldsymbol{\Phi}) \times m \boldsymbol{v} .
\end{aligned}
$$


The term with the equation for conservation of momentum reduces to zero. Also, the cross product of a vector with itself results in a null vector. Therefore, the equation for conservation of angular momentum relative to the particle position becomes

$$
\begin{aligned}
& \frac{\mathrm{d}}{\mathrm{d} t} \iint_{V_{\mathrm{p}}} \int_{\boldsymbol{\chi}} \boldsymbol{\chi} \times \boldsymbol{u} \mathrm{d} V=\iint_{\partial V_{\mathrm{p}}} \boldsymbol{\chi} \times(\boldsymbol{\sigma} \cdot \boldsymbol{n}) \mathrm{d} S+\iiint_{V_{\mathrm{p}}} \rho \boldsymbol{\chi} \times \boldsymbol{f} \mathrm{d} V \\
& +\iint_{\partial V_{\mathrm{p}}} \phi \boldsymbol{\chi} \times \boldsymbol{u} \mathrm{d} S+m \boldsymbol{v} \times \boldsymbol{\Phi}
\end{aligned}
$$





\section{APPENDiX B}

\section{INTEGRALS FOR SPHERICAL VOLUME}

\section{B.1 Volume of a sphere}

For a spherical particle, volume integrals have to be evaluated. First the volume will be calculated by integrating 1 over the spherical volume

$$
V_{\mathrm{p}} \equiv \iiint_{V_{\mathrm{sphere}}} \mathrm{d} V
$$

In Cartesian coordinates for a spherical particle with diameter $R$, equation (B.1) is evaluated as

$$
\iiint_{V_{\mathrm{sphere}}} \mathrm{d} V=\int_{-R}^{R}\left\{\int_{-\sqrt{R^{2}-z^{2}}}^{\sqrt{R^{2}-z^{2}}}\left\{\int_{-\sqrt{R^{2}-y^{2}-z^{2}}}^{\sqrt{R^{2}-y^{2}-z^{2}}} \mathrm{~d} x\right\} d y\right\} d z .
$$

Evaluation of the innermost integral leads to

$$
\iiint_{V_{\text {sphere }}} \mathrm{d} V=\int_{-R}^{R}\left\{\int_{-\sqrt{R^{2}-z^{2}}}^{\sqrt{R^{2}-z^{2}}}\left\{2 \sqrt{R^{2}-y^{2}-z^{2}}\right\} d y\right\} d z .
$$




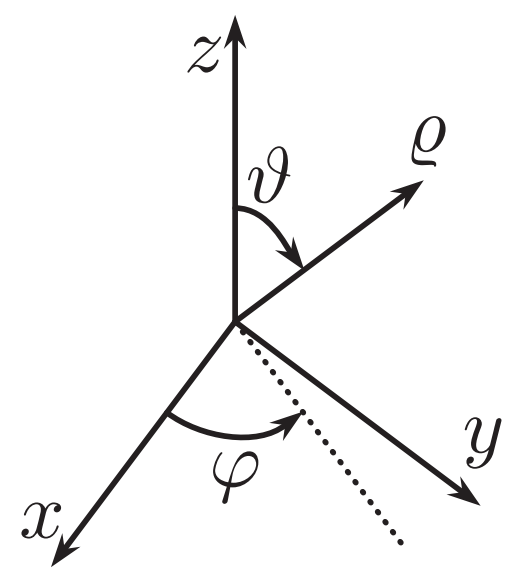

Figure B.1: The Cartesian and spherical coordinate systems

Subsequently leading to

$$
\iiint_{V_{\text {sphere }}} \mathrm{d} V=\int_{-R}^{R}\left\{R^{2}-z^{2}\right\} \pi d z .
$$

Which finally results in the volume of the particle

$$
\iiint_{V_{\text {sphere }}} \mathrm{d} V=\frac{4}{3} \pi R^{3}
$$

or

$$
\iiint_{V_{\text {sphere }}} \mathrm{d} V=\frac{\pi}{6} D^{3}
$$

with $D$ the diameter of the particle.

\section{B.2 From Cartesian to spherical}

In the preceding derivation, Cartesian coordinates were used, resulting in complicated integral bounds. Transformation to a spherical coordinate system would 
decrease the complexity of the boundaries, at the cost of a transformation of the integral. The spherical coordinate system, with coordinates

$$
\boldsymbol{r} \equiv(\varrho, \vartheta, \varphi),
$$

is shown in figure B.1. The coordinates in Cartesian coordinates are then related to the coordinates in the spherical coordinate system by

$$
\begin{aligned}
& x=\varrho \sin \vartheta \cos \varphi, \\
& y=\varrho \sin \vartheta \sin \varphi, \\
& z=\varrho \cos \vartheta .
\end{aligned}
$$

For the coordinate transformation, the derivatives of the Cartesian coordinates to the spherical coordinates are required. The Jacobian matrix becomes

$$
\frac{\partial \boldsymbol{x}}{\partial \boldsymbol{r}} \equiv\left[\begin{array}{lll}
\frac{\partial x}{\partial \varrho} & \frac{\partial y}{\partial \varrho} & \frac{\partial z}{\partial \varrho} \\
\frac{\partial x}{\partial \vartheta} & \frac{\partial y}{\partial \vartheta} & \frac{\partial z}{\partial \vartheta} \\
\frac{\partial x}{\partial \varphi} & \frac{\partial y}{\partial \varphi} & \frac{\partial z}{\partial \varphi}
\end{array}\right]
$$

The integral in the spherical coordinate system is related to the Cartesian coordinate system by the determinant of the Jacobian matrix

$$
\iiint_{V_{x, y, z}} f_{x, y, z} \mathrm{~d} V_{x, y, z} \equiv \iiint_{V_{\varrho, \vartheta, \varphi}} f_{\varrho, \vartheta, \varphi}\left|\left(\frac{\partial \boldsymbol{x}}{\partial \boldsymbol{r}}\right)^{\mathrm{T}}\right| \mathrm{d} V_{\varrho, \vartheta, \varphi} .
$$

Therefore, for the spherical coordinate system the relation to the volume integral for Cartesian coordinates becomes

$$
\iiint_{V_{x, y, z}} f_{x, y, z} \mathrm{~d} V_{x, y, z}=\iiint_{V_{\varrho}, \vartheta, \varphi} f_{\varrho, \vartheta, \varphi} \varrho^{2} \sin \vartheta \mathrm{d} V_{\varrho, \vartheta, \varphi} .
$$

For a sphere the integral borders are much more convenient than the borders for the Cartesian coordinate system. The integral for the volume of a sphere becomes

$$
\iiint_{V_{\text {sphere }}} \mathrm{d} V=\int_{0}^{R}\left\{\int_{0}^{\pi}\left\{\int_{0}^{2 \pi} \varrho^{2} \sin \vartheta \mathrm{d} \varphi\right\} d \vartheta\right\} d \varrho,
$$

which can be evaluated easily to acquire the same result as for the Cartesian coordinate system, equation (B.4). As it is more convenient to evaluate the integrals in spherical coordinates for spherical volumes, subsequent integrals will be evaluated in spherical coordinates. 


\section{B.3 Surface of a sphere}

The integral for the surface of a sphere is easily obtained from the integral for the volume of a sphere in equation (B.10). The only difference will be that the integration in radial direction is not performed and $\varrho$ is replaced by $R$. This results in

$$
\iint_{\partial V_{\text {sphere }}} \mathrm{d} S=\int_{0}^{\pi}\left\{\int_{0}^{2 \pi} R^{2} \sin \vartheta \mathrm{d} \varphi\right\} d \vartheta
$$

Evaluation of the integrals leads to

$$
\left.\iint_{\partial V_{\text {sphere }}} \mathrm{d} S=R^{2}[-\cos \vartheta\}\right]\left.\left.\right|_{0} ^{\pi}[\varphi]\right|_{0} ^{2 \pi},
$$

and therefore

$$
\iint_{\partial V_{\text {sphere }}} \mathrm{d} S=4 \pi R^{2}
$$

or

$$
\iint_{\partial V_{\text {sphere }}} \mathrm{d} S=\pi D^{2}
$$

\section{B.4 First moment for a sphere}

The first moment in spherical coordinates is

$$
\iiint_{V_{\text {sphere }}} \boldsymbol{x} \mathrm{d} V=\int_{0}^{R} \int_{0}^{\pi} \int_{0}^{2 \pi}\left(\begin{array}{c}
\sin \vartheta \cos \varphi \\
\sin \vartheta \sin \varphi \\
\cos \vartheta
\end{array}\right) \varrho^{3} \sin \vartheta \mathrm{d} \varphi d \vartheta d \varrho
$$

and thus

$$
\iiint_{V_{\text {sphere }}} \boldsymbol{x} \mathrm{d} V=\int_{0}^{R} \int_{0}^{\pi}\left(\begin{array}{c}
\left.\sin \vartheta[\sin \varphi]\right|_{0} ^{2 \pi} \\
\left.\sin \vartheta[-\cos \varphi]\right|_{0} ^{2 \pi} \\
\left.\cos \vartheta[\varphi]\right|_{0} ^{2 \pi}
\end{array}\right) \varrho^{3} \sin \vartheta d \vartheta d \varrho .
$$

which results in

$$
\iiint_{V_{\text {sphere }}} \boldsymbol{x} \mathrm{d} V=\int_{0}^{R}\left(\begin{array}{c}
0 \\
0 \\
\left.2 \pi \varrho^{3}\left[\sin ^{2} \vartheta\right]\right|_{0} ^{\pi}
\end{array}\right) d \varrho .
$$


Therefore, the first moment is

$$
\iiint_{V_{\text {sphere }}} \boldsymbol{x} \mathrm{d} V=\mathbf{0}
$$

Moreover, the first moment over the surface of the sphere, using the result of equation (B.17), is

$$
\underset{\partial V_{\text {sphere }}}{\int} \boldsymbol{x} \mathrm{d} V=\mathbf{0}
$$

It has to be noted that for all higher order moments, the odd moments are equal to zero, as each point in one octant has a partner point of equal magnitude but different sign in the octant point-symmetric with respect to the centre of the sphere.

\section{B.5 Second moment for a sphere}

The second moment with $\boldsymbol{x} \boldsymbol{x}$ involves a $3 \times 3$ matrix. For readability $\boldsymbol{x} x, \boldsymbol{x} y$ and $\boldsymbol{x} z$ will be evaluated seperately. In spherical coordinates the first column of the second moment is

$$
\iiint_{V_{\text {sphere }}} \boldsymbol{x} x \mathrm{~d} V=\int_{0}^{R} \int_{0}^{\pi} \int_{0}^{2 \pi}\left(\begin{array}{c}
\sin ^{3} \vartheta \cos ^{2} \varphi \\
\sin ^{3} \vartheta \sin \varphi \cos \varphi \\
\cos \vartheta \sin ^{2} \vartheta \cos \varphi
\end{array}\right) \varrho^{4} \mathrm{~d} \varphi d \vartheta d \varrho .
$$

First the trigonometric identity $\cos 2 \varphi=2 \cos ^{2} \varphi-1$ is applied to $\cos ^{2} \varphi$. This results in

$$
\iiint_{V_{\text {sphere }}} \boldsymbol{x} x \mathrm{~d} V=\int_{0}^{R} \int_{0}^{\pi} \int_{0}^{2 \pi}\left(\begin{array}{c}
\sin ^{3} \vartheta \frac{1}{2}[1+\cos 2 \varphi] \\
\sin ^{3} \vartheta \sin \varphi \cos \varphi \\
\cos \vartheta \sin ^{2} \vartheta \cos \varphi
\end{array}\right) \varrho^{4} \mathrm{~d} \varphi d \vartheta d \varrho .
$$

Evaluation of the integral over $\varphi$ results in

$$
\iiint_{V_{\text {sphere }}} \boldsymbol{x} x \mathrm{~d} V=\int_{0}^{R} \int_{0}^{\pi}\left(\begin{array}{c}
\left.\sin ^{3} \vartheta\left[\frac{\varphi}{2}+\frac{1}{4} \sin 2 \varphi\right]\right|_{0_{0}^{2 \pi}} ^{2 \pi} \\
\left.\sin ^{3} \vartheta\left[\frac{1}{2} \sin ^{2} \varphi\right]\right|_{0} ^{2 \pi} \\
\left.\cos \vartheta \sin ^{2} \vartheta[\sin \varphi]\right|_{0} ^{2 \pi}
\end{array}\right) \varrho^{4} d \vartheta d \varrho .
$$


Subsequently, the trigonometric identity $\sin ^{2} \vartheta+\cos ^{2} \vartheta=1$ is applied to $\sin ^{3} \vartheta$, resulting in

$$
\iiint_{V_{\text {sphere }}} \boldsymbol{x} x \mathrm{~d} V=\int_{0}^{R} \int_{0}^{\pi}\left(\begin{array}{c}
\pi \sin \vartheta\left[1-\cos ^{2} \vartheta\right] \\
0 \\
0
\end{array}\right) \varrho^{4} d \vartheta d \varrho,
$$

and thus

$$
\iiint_{V_{\text {sphere }}} \boldsymbol{x} x \mathrm{~d} V=\int_{0}^{R}\left(\begin{array}{c}
\left.\pi\left[\frac{1}{3} \cos ^{3} \vartheta-\cos \vartheta\right]\right|_{0} ^{\pi} \\
0 \\
0
\end{array}\right) \varrho^{4} d \varrho .
$$

Therefore,

$$
\iiint_{V_{\text {sphere }}} \boldsymbol{x} x \mathrm{~d} V=\left.\left(\begin{array}{c}
\frac{4}{3} \pi \\
0 \\
0
\end{array}\right)\left[\frac{\varrho^{5}}{5}\right]\right|_{0} ^{R}
$$

The second column of the second moment is

$$
\iiint_{V_{\text {sphere }}} \boldsymbol{x} y \mathrm{~d} V=\int_{0}^{R} \int_{0}^{\pi} \int_{0}^{2 \pi}\left(\begin{array}{c}
\sin ^{3} \vartheta \cos \varphi \sin \varphi \\
\sin ^{3} \vartheta \sin ^{2} \varphi \\
\cos \vartheta \sin ^{2} \vartheta \sin \varphi
\end{array}\right) \varrho^{4} \mathrm{~d} \varphi d \vartheta d \varrho .
$$

Now first the trigonometric identity $\cos 2 \varphi=1-2 \sin ^{2} \varphi$ is applied to $\sin ^{2} \varphi$. Subsequently, the trigonometric identity $\sin ^{2} \vartheta+\cos ^{2} \vartheta=1$ is applied to $\sin ^{3} \vartheta$. Evaluation of the integrals leads, in a similar way as for the first column to

$$
\iiint_{V_{\text {sphere }}} \boldsymbol{x} y \mathrm{~d} V=\int_{0}^{R}\left(\begin{array}{c}
0 \\
\frac{4}{3} \pi \\
0
\end{array}\right) \varrho^{4} d \varrho .
$$

Finally the last column is

$$
\iiint_{V_{\text {sphere }}} \boldsymbol{x} z \mathrm{~d} V=\int_{0}^{R} \int_{0}^{\pi} \int_{0}^{2 \pi}\left(\begin{array}{c}
\sin ^{2} \vartheta \cos \vartheta \cos \varphi \\
\sin ^{2} \vartheta \cos \vartheta \sin \varphi \\
\cos ^{2} \vartheta \sin \vartheta
\end{array}\right) \varrho^{4} \mathrm{~d} \varphi d \vartheta d \varrho,
$$

resulting in

$$
\iiint_{V_{\text {sphere }}} \boldsymbol{x} z \mathrm{~d} V=\int_{0}^{R}\left(\begin{array}{c}
0 \\
0 \\
\frac{4}{3} \pi
\end{array}\right) \varrho^{4} d \varrho
$$


Equations (B.24), (B.27) and (B.29) will now be taken together. Moreover, the evaluation of the integral in equation (B.24) as shown in equation (B.25) will be applied to the other columns as well. This results in the second moment for a sphere

$$
\iiint_{V_{\text {sphere }}} \boldsymbol{x} \boldsymbol{x} \mathrm{d} V=\frac{4}{15} \pi R^{5} \mathbf{U}
$$

or

$$
\iiint_{V_{\text {sphere }}} \boldsymbol{x} \boldsymbol{x} \mathrm{d} V=\frac{\pi}{120} D^{5} \mathbf{U} .
$$

For the second moment over the surface of a sphere, the results in equations (B.24), (B.27) and (B.29) can be used leading to

$$
\iint_{\partial V_{\text {sphere }}} \boldsymbol{x} \boldsymbol{x} \mathrm{d} V=\frac{4}{3} \pi R^{4} \mathbf{U},
$$

or

$$
\iint_{\partial V_{\text {sphere }}} \boldsymbol{x} \boldsymbol{x} \mathrm{d} V=\frac{\pi}{12} D^{4} \mathbf{U} .
$$

\section{B.6 Moment of inertia}

\section{B.6.1 A uniform sphere}

The moment of inertia for a sphere of uniform density, using the definition in equation (2.18) with the coordinate system coinciding with the centre of the sphere, is

$$
\mathbf{I}_{\text {sphere }} \equiv \rho \iiint_{V_{\mathrm{p}}}\left(\|\boldsymbol{x}\|^{2} \mathbf{U}-\boldsymbol{x} \boldsymbol{x}\right) d V .
$$

The results from the previous section, and more specifically equation (B.31), can be used to evaluate the moment of inertia for a sphere, resulting in

$$
\mathbf{I}_{\text {sphere }}=\frac{8}{15} \pi \rho R^{5} \mathbf{U}
$$


Using the result of equation (B.3) for the volume of the sphere the moment of inertia can be expressed in terms of the mass and the radius of the sphere

$$
\mathbf{I}_{\text {sphere }}=\frac{2}{5} m R^{2} \mathbf{U}
$$

or

$$
\mathbf{I}_{\text {sphere }}=\frac{1}{10} m D^{2} \mathbf{U}
$$

\section{B.6.2 A uniform spherical shell}

The moment of inertia for a spherical shell of uniform density, with outer radius $R_{1}$ and inner radius $R_{0}$ is calculated similar to the moment of inertia for a uniform sphere. The results from the previous section, and more specifically equations (B.24), (B.27) and (B.29), as well as the result of equation (B.35), can be used to evaluate the moment of inertia for a spherical shell, resulting in

$$
\mathbf{I}_{\text {shell }}=\frac{8}{15} \pi \rho\left(R_{1}^{5}-R_{0}^{5}\right) \mathbf{U}
$$

Using the result of equation (B.3) for the volume of the sphere the moment of inertia can be expressed in terms of the mass and the radius of the sphere

$$
\mathbf{I}_{\text {shell }}=\frac{2}{5} m \frac{R_{1}^{5}-R_{0}^{5}}{R_{1}^{3}-R_{0}^{3}} \mathbf{U}
$$

or

$$
\mathbf{I}_{\text {shell }}=\frac{1}{10} m \frac{D_{1}^{5}-D_{0}^{5}}{D_{1}^{3}-D_{0}^{3}} \mathbf{U} .
$$

Moreover, if the thickness of the shell is much smaller than the radius, the outer radius can be expressed as $R_{1}=R_{0}+\epsilon$, where $\epsilon \ll R_{0}$. Equations (B.39) and (B.40) then reduce to

$$
\mathbf{I}_{\text {shell }} \approx \frac{2}{3} m R_{0}^{2} \mathbf{U}
$$

or

$$
\mathbf{I}_{\text {shell }} \approx \frac{1}{6} m D_{0}^{2} \mathbf{U}
$$




\section{B.6.3 A uniform sphere with a uniform shell}

In case of flotation, the particle exists of two distinct regions that can be approximated by a sphere of mass $m_{0}$ with radius $R_{0}$, and a shell of mass $m_{1}$ and outer radius $R_{1}$. The moment of inertia then becomes a combination of the results of equations (B.36) and (B.39).

$$
\mathbf{I}_{\text {sphere }+ \text { shell }}=\frac{2}{5}\left(m_{0} R_{0}^{2}+m_{1} \frac{R_{1}^{5}-R_{0}^{5}}{R_{1}^{3}-R_{0}^{3}}\right) \mathbf{U},
$$

or

$$
\mathbf{I}_{\text {sphere+shell }}=\frac{1}{10}\left(m_{0} D_{0}^{2}+m_{1} \frac{D_{1}^{5}-D_{0}^{5}}{D_{1}^{3}-D_{0}^{3}}\right) \mathbf{U} .
$$

For a thin shell about a relatively large sphere, this results in

$$
\mathbf{I}_{\text {sphere }+ \text { shell }} \approx \frac{2}{5}\left(m_{0}+\frac{5}{3} m_{1}\right) R_{0}^{2} \mathbf{U}
$$

or

$$
\mathbf{I}_{\text {sphere+shell }} \approx \frac{1}{10}\left(m_{0}+\frac{5}{3} m_{1}\right) D_{0}^{2} \mathbf{U} .
$$

\section{B.7 Fourth moment for a sphere}

Making continuous use of the trigonometric identities, the fourth moment for a sphere becomes

$$
\begin{aligned}
& \iiint_{V_{\text {sphere }}} \boldsymbol{x} \boldsymbol{x} x \boldsymbol{d} \mathrm{d} V=\left[\begin{array}{lll}
3 & 0 & 0 \\
0 & 1 & 0 \\
0 & 0 & 1
\end{array}\right] \frac{4 \pi}{105} R^{7}, \\
& \iiint_{V_{\text {sphere }} \boldsymbol{x} \boldsymbol{x} y y \mathrm{~d} V}=\left[\begin{array}{lll}
1 & 0 & 0 \\
0 & 3 & 0 \\
0 & 0 & 1
\end{array}\right] \frac{4 \pi}{105} R^{7}, \\
& \iiint_{V_{\text {sphere }}} \boldsymbol{x} \boldsymbol{x} z z \mathrm{~d} V=\left[\begin{array}{lll}
1 & 0 & 0 \\
0 & 1 & 0 \\
0 & 0 & 3
\end{array}\right] \frac{4 \pi}{105} R^{7},
\end{aligned}
$$

or

$$
\iiint_{V_{\text {sphere }}} \boldsymbol{x} \boldsymbol{x} x x \mathrm{~d} V=\left[\begin{array}{lll}
3 & 0 & 0 \\
0 & 1 & 0 \\
0 & 0 & 1
\end{array}\right] \frac{\pi}{3360} D^{7}
$$




$$
\begin{array}{|l}
\iiint_{V_{\text {sphere }}} \boldsymbol{x} \boldsymbol{x} y y \mathrm{~d} V=\left[\begin{array}{lll}
1 & 0 & 0 \\
0 & 3 & 0 \\
0 & 0 & 1
\end{array}\right] \frac{\pi}{3360} D^{7}, \\
\iiint_{V_{\text {sphere }}} \boldsymbol{x} \boldsymbol{x} z z \mathrm{~d} V=\left[\begin{array}{lll}
1 & 0 & 0 \\
0 & 1 & 0 \\
0 & 0 & 3
\end{array}\right] \frac{\pi}{3360} D^{7} .
\end{array}
$$

The surface integrals for the fourth moment for a sphere become

$$
\begin{aligned}
\iint_{\partial V_{\text {sphere }} \boldsymbol{x} \boldsymbol{x} x x \mathrm{~d} S} & =\left[\begin{array}{lll}
3 & 0 & 0 \\
0 & 1 & 0 \\
0 & 0 & 1
\end{array}\right] \frac{4 \pi}{15} R^{6}, \\
\iint_{\partial V_{\text {sphere }}} \boldsymbol{x} \boldsymbol{x} y y \mathrm{~d} S & =\left[\begin{array}{lll}
1 & 0 & 0 \\
0 & 3 & 0 \\
0 & 0 & 1
\end{array}\right] \frac{4 \pi}{15} R^{6}, \\
\iint_{\partial V_{\text {sphere }}} \boldsymbol{x} \boldsymbol{x} z z \mathrm{~d} S & =\left[\begin{array}{lll}
1 & 0 & 0 \\
0 & 1 & 0 \\
0 & 0 & 3
\end{array}\right] \frac{4 \pi}{15} R^{6},
\end{aligned}
$$

or

$$
\underset{\partial V_{\text {sphere }}}{\iint_{\text {a }} \boldsymbol{x} x x} \mathrm{~d} S=\left[\begin{array}{lll}
3 & 0 & 0 \\
0 & 1 & 0 \\
0 & 0 & 1
\end{array}\right] \frac{\pi}{240} D^{6},
$$

$$
\iint_{\partial V_{\text {sphere }}} \boldsymbol{x} \boldsymbol{x} y y \mathrm{~d} S=\left[\begin{array}{lll}
1 & 0 & 0 \\
0 & 3 & 0 \\
0 & 0 & 1
\end{array}\right] \frac{\pi}{240} D^{6}
$$

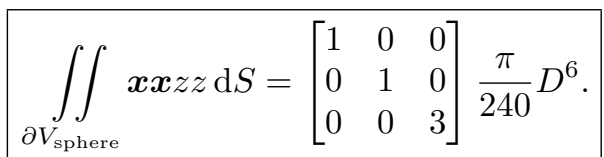




\section{B.8 List of moments}

The resulting moments derived in this chapter are summarized in table B.1. Moreover, the moments in terms of surface and volume have been presented in table B.2. 


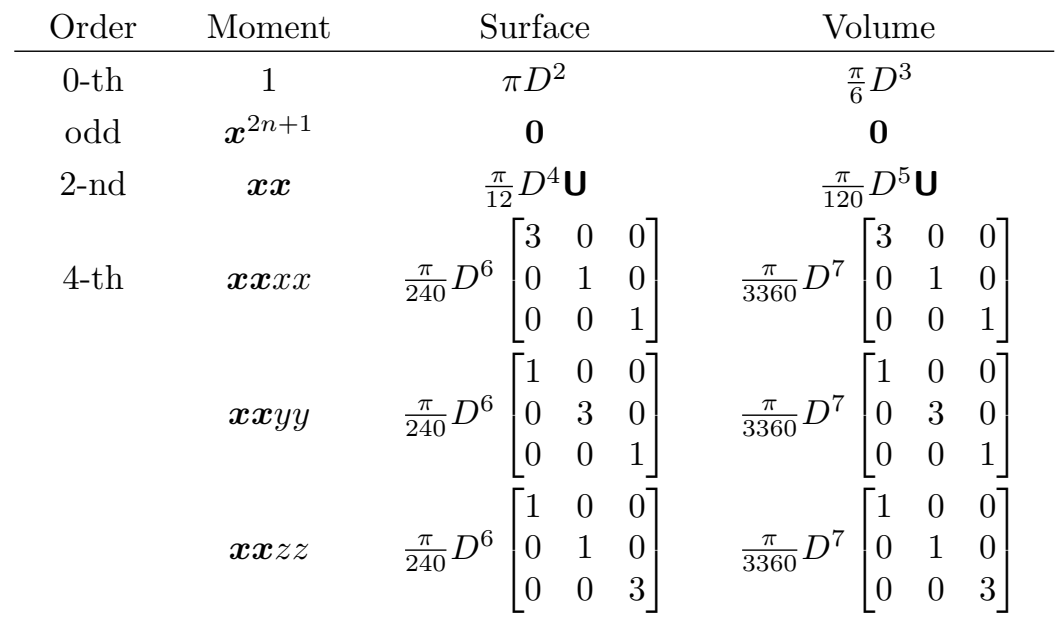

Table B.1: The moments for a sphere, derived in this section.

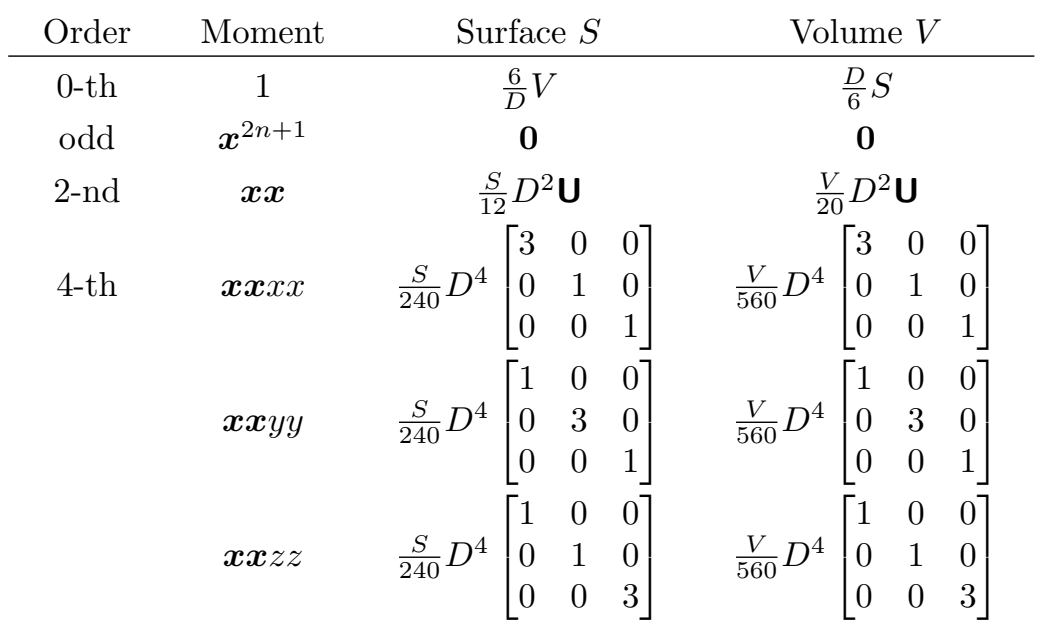

Table B.2: The moments for a sphere in terms of particle surface and volume. 


\section{ACKNOWLEDGEMENTS}

This research was performed for the FACE centre, a research cooperation between IFE, NTNU, and SINTEF. The centre is funded by The Research Council of Norway, and by the following industrial partners: Statoil, Scandpower Petroleum Technology AS, FMC, CD-Adapco, and Shell Technology Norway AS. The research has been performed at the University of Twente.

I would like to thank the University of Twente, FMC Technologies and the FACE centre for giving me the opportunity to perform my $\mathrm{PhD}$ research on this topic. I would also like to thank Harry Hoeijmakers of the EFD group of the University of Twente for introducing me to FMC Technologies and supervising me during my research. Furthermore I would like to thank Rune Fantoft and Reda Akdim and Paul Verbeek for allowing me to work on this project, and Remko Westra, Thomas Krebs and Govert Kruijtzer for their input in my project.

At the FACE centre I always had a warm welcome. I would like to thank Martin Foss, Kristian Sveen, Gustavo Zarruk and Olaf Skjaeraasen for organizing these meetings and for the pleasant conversations and discussions.

My stay at the EFD group was always pleasant. I had a great time, especially with my former roommates Jesse, Koen and Faraz. During coffee-breaks and social drinks Sietse was always there, and we had a lot interesting and funny discussions. Anjenet, thank you for adding fun to the coffee-breaks. Harry, I was always happy to work with you and your group with such a nice atmosphere. I always enjoyed our discussions and traveling with you to meetings and conferences. Kees, it was always nice to get back to the multi-grid subject and I hope you can keep up the topics on that part. I would also like to thank all the other students, PhD students, post-docs and staff for my pleasant stay, the nice discussions and their enthusiasm about their research.

During my time at the UT I met a lot of people especially during spinning and running. Tjerk, thank you for giving all those great spinning lessons. Jorrit, 
Fjodor, Mieke, Frederik and Julia thank you for letting me run with you, and organizing the Horsthollers BATA-team. Jorrit, thanks for all the nice climbs at Gildehaus and all the running and spinning.

I had a nice time and a pleasant stay at Matenweg 32 and I cannot mention all people I would like to thank. Kasper, Bart Niels and Enne thanks for all the fun with our D\&D sessions, that made my time so nice.

Jan en Marijke, thank you for letting me become who I am now. I hope you did not have to worry too much about me getting the work finished. Jan, thank you for all the feedback on the thesis. Karel, special thanks for helping me move twice during the years I was doing this research.

After my contract ended, I immediately started working at VORtech. Being surrounded by nice people, I was able to have a very flexible time-schedule in order to work on my thesis efficiently. Special thanks to Mark and Bas for allowing me to do that and to Marieke for the extra work administrating that.

During my PhD study, the happiest moment of all was me meeting Anna. She has not only made me happy but also more confident and relaxed. It was always hard to leave you after a weekend, but I could always look forward to seeing you again. Thank you for being here with me. 


\section{ABOUt THE AUTHOR}

Dirk van Eijkeren was born in Leidschendam, the Netherlands, on 1 July 1984.

In 2002 he started with his Bachelors education on Mechanical Engineering at the University of Twente, including a minor Aeronautical Engineering. After obtaining his Bachelors degree in 2006, he continued with his Masters education on Mechanical Engineering at the University of Twente, with a specialization in Engineering Fluid Dynamics. He graduated at the Engineering Fluid Dynamics group in 2008 with his masters research "Multi-Level Wave-Ray Solution of the 2D-Helmholtz Equation".

After graduation, Dirk started presented PhD research in 2009, resulting in four articles in proceedings $[17,18,19,20]$ and this thesis. In 2013, he started working in his current position as scientific software engineer at VORtech BV.

Dirks current position involves seismic wave propagation, contact mechanics and elasticity as well as applied mathematics in general. He is also interested in multi-grid and the Lattice Boltzmann method. 